SUPPORTING INFORMATION

\title{
SOMO-HOMO Conversion in Triplet Cyclopentane-1,3-diyl Diradicals
}

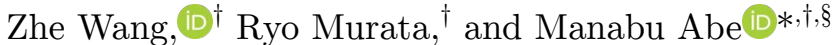

${ }^{\dagger}$ Department of Chemistry, Graduate School of Science, Hiroshima University, 1-3-1 Kagamiyama, HigashiHiroshima, Hiroshima 739-8526, Japan

${ }^{\S}$ Hiroshima University Research Center for Photo-Drug-Delivery-Systems (HiU-P-DDS), Hiroshima University, 1-3-1 Kagamiyama, Higashi-Hiroshima, Hiroshima 739-8526, Japan

*E-mail: mabe@hiroshima-u.ac.jp

\section{Table of Contents}

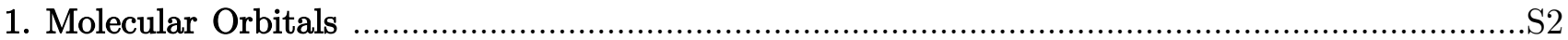

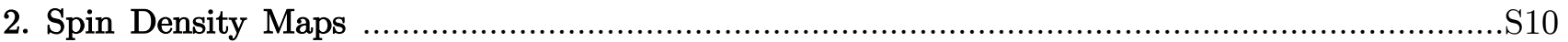

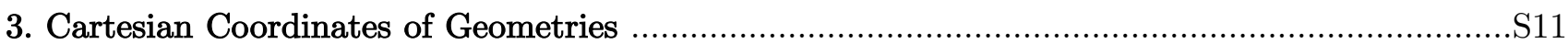

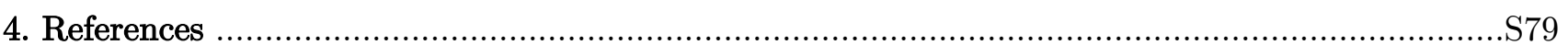




\section{Molecular Orbitals}

Molecular orbitals of DR1-5 calculated at UB3LYP/6-31G(d) (for DR3F1': UB3LYP/6-31+G(d)) level of theory are visualized with an iso-surface value of 0.02 a.u. using GaussView 6.0.16 program ${ }^{[\mathrm{S} 1]}$. Selected molecular orbitals are listed in Figure S1 $\sim$ S8.

(a)

171
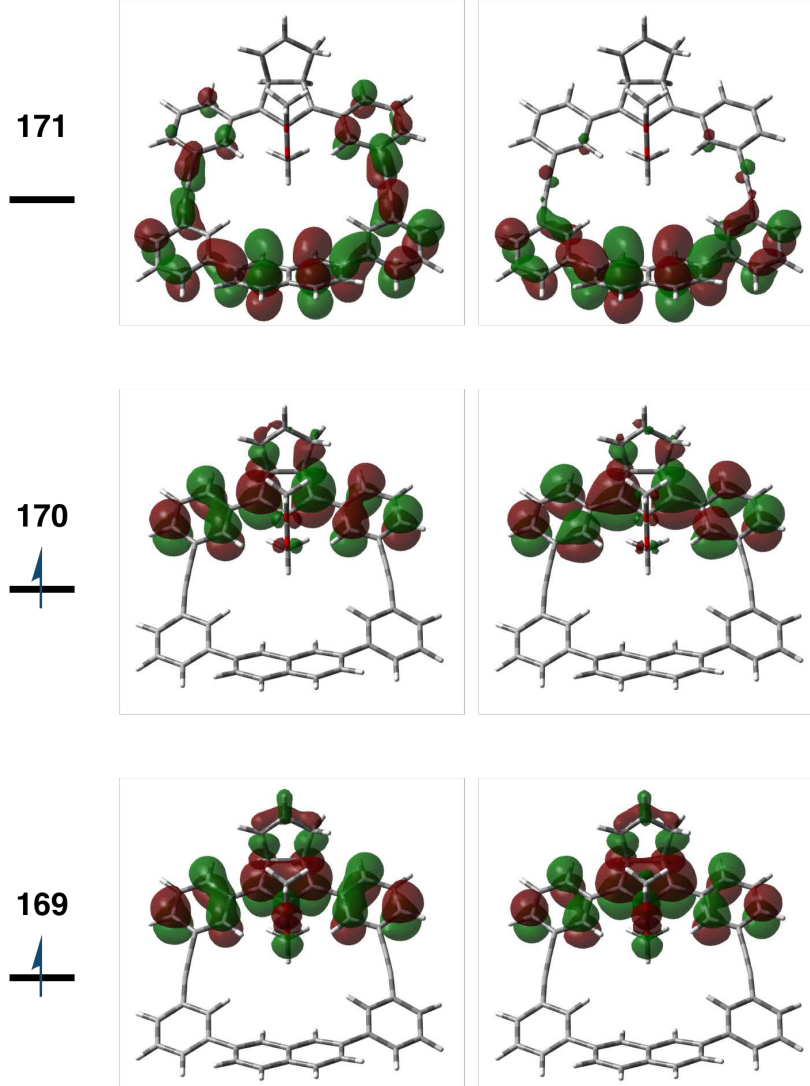

168
$\frac{4}{4}$
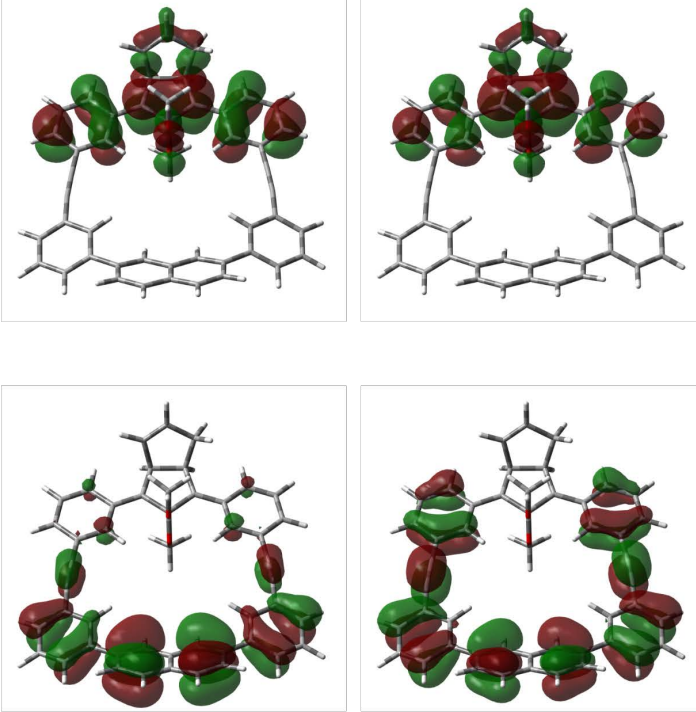

167
4
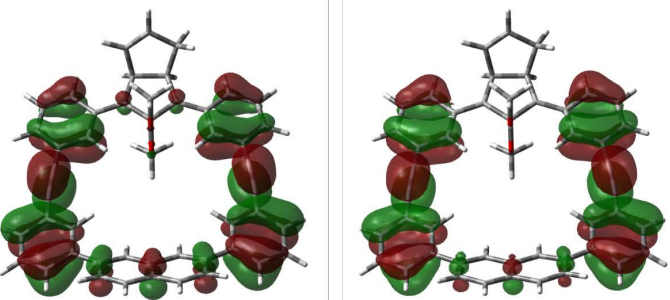

1
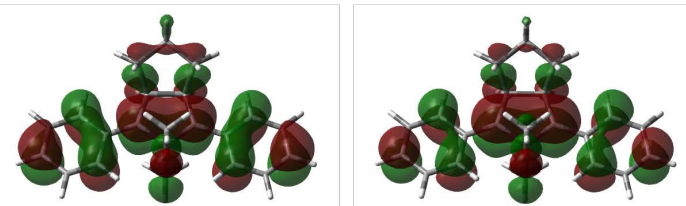

85
4
41
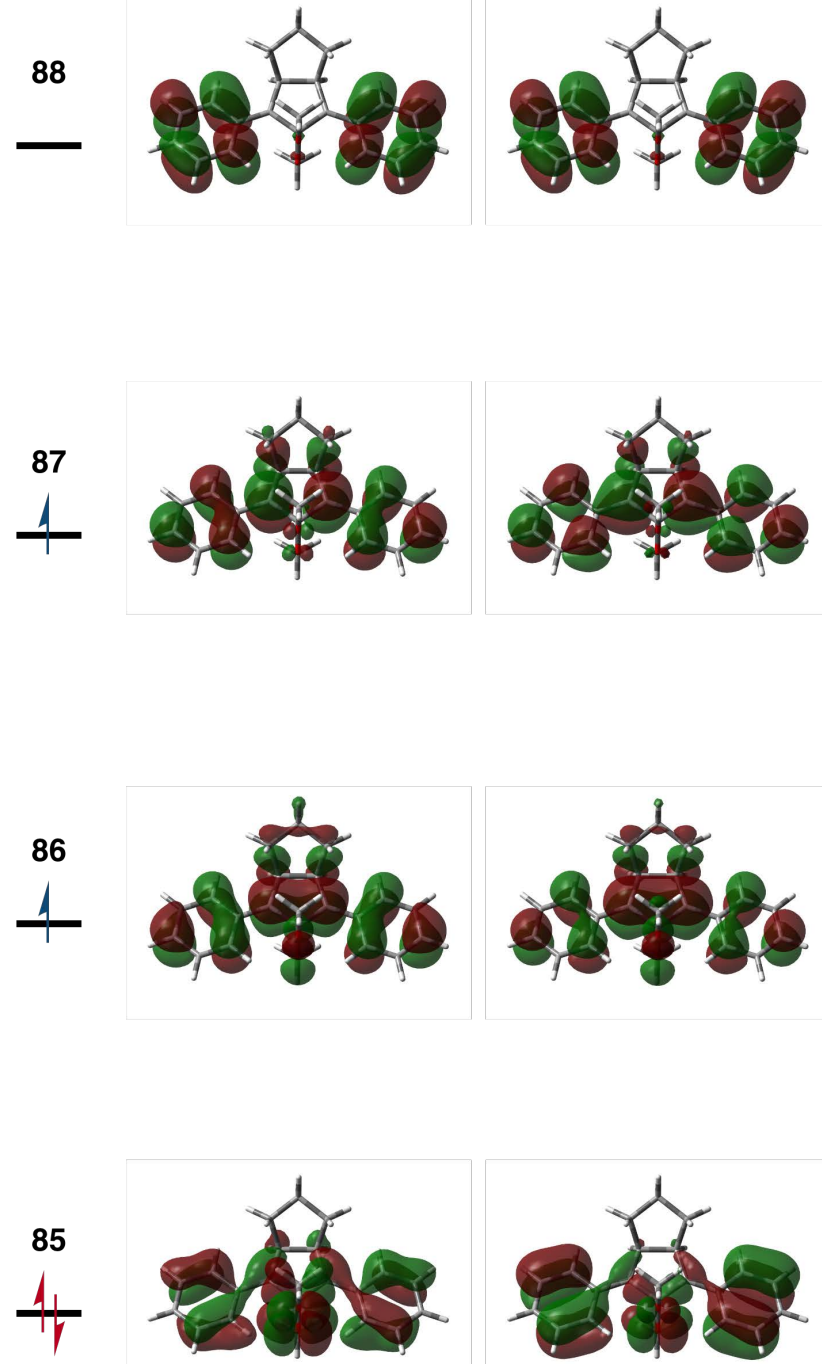

\begin{tabular}{l}
87 \\
4 \\
\hline
\end{tabular}

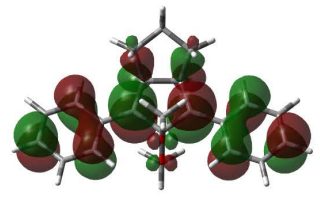

res

84
4
41
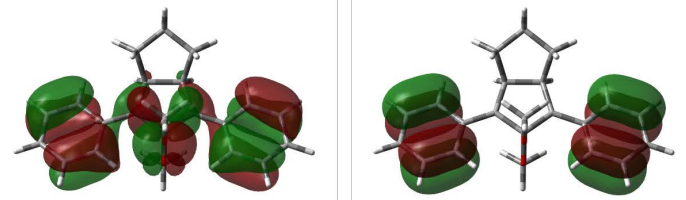

Figure S1. Selected molecular orbitals of (a) DR1 and (b) DR2. 
(a)
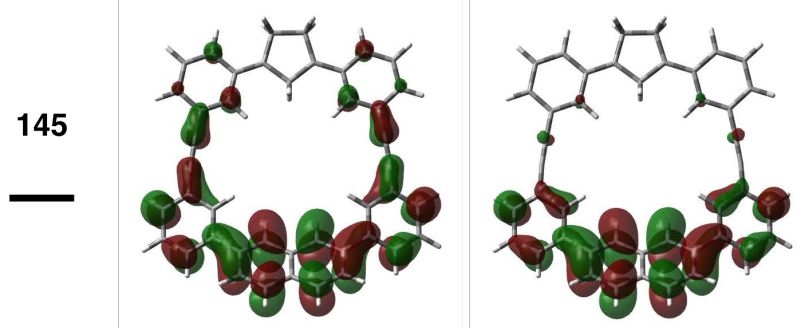

\begin{tabular}{c}
144 \\
- \\
\hline
\end{tabular}
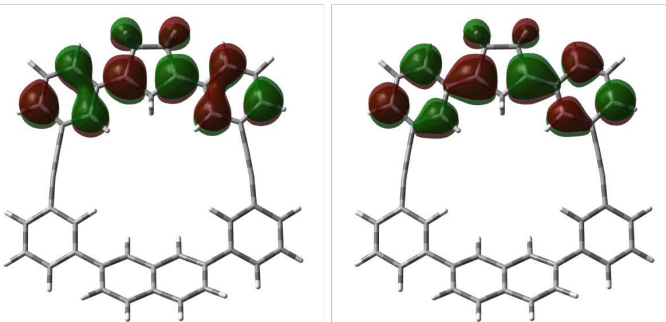

143

4
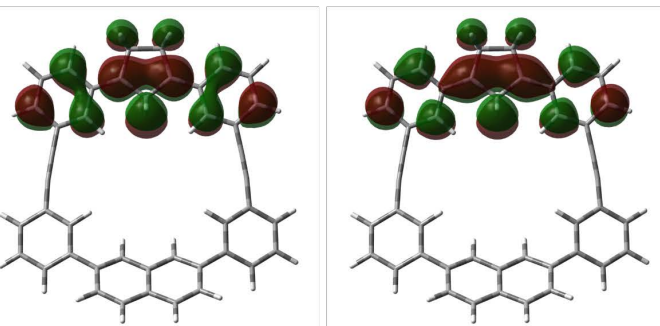

142

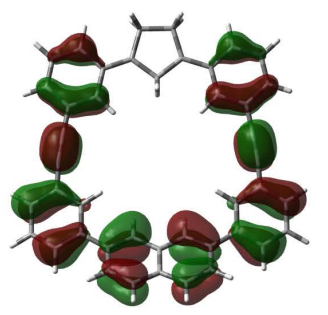

150
4
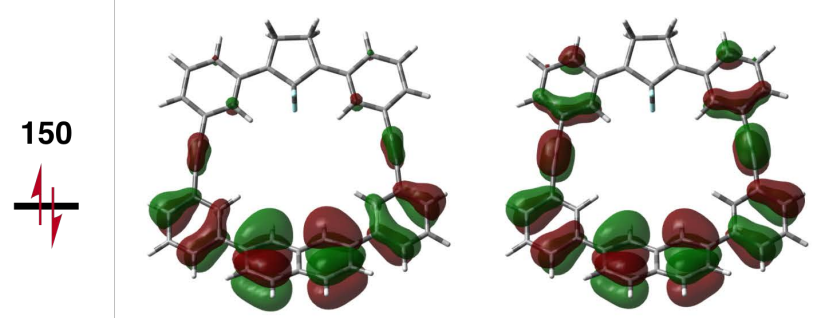

151
4
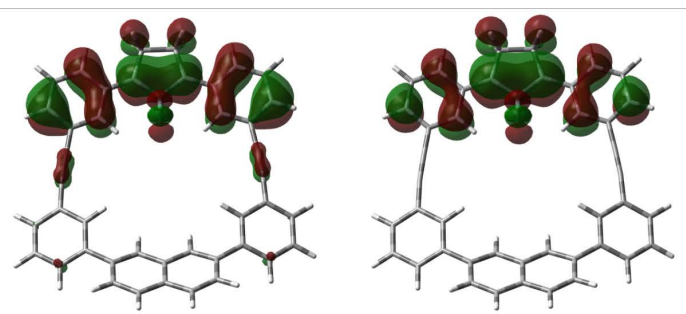

152

1
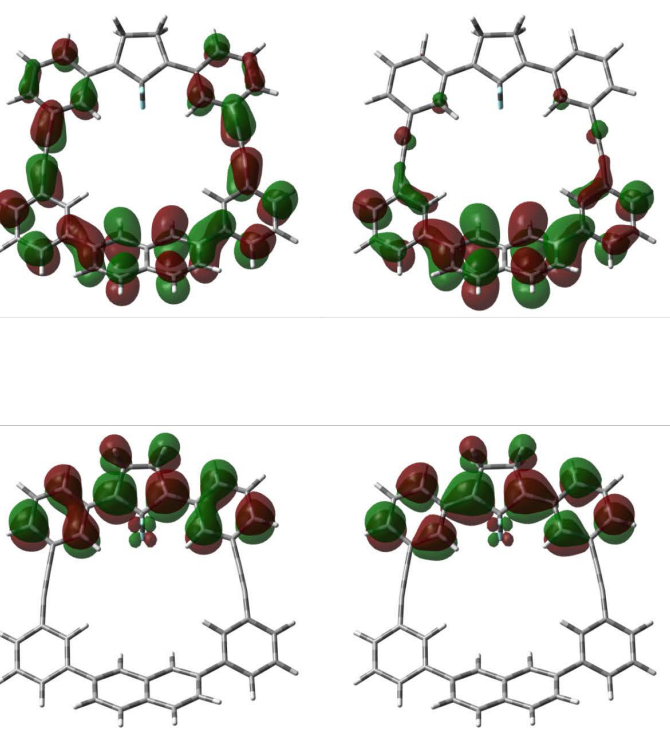

\begin{tabular}{c}
149 \\
4 \\
\hline 17
\end{tabular}

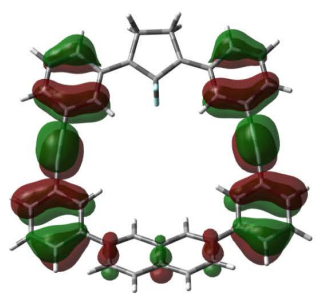

Figure S2. Selected molecular orbitals of (a) DR3H0 and (b) DR3F0. 
(a)
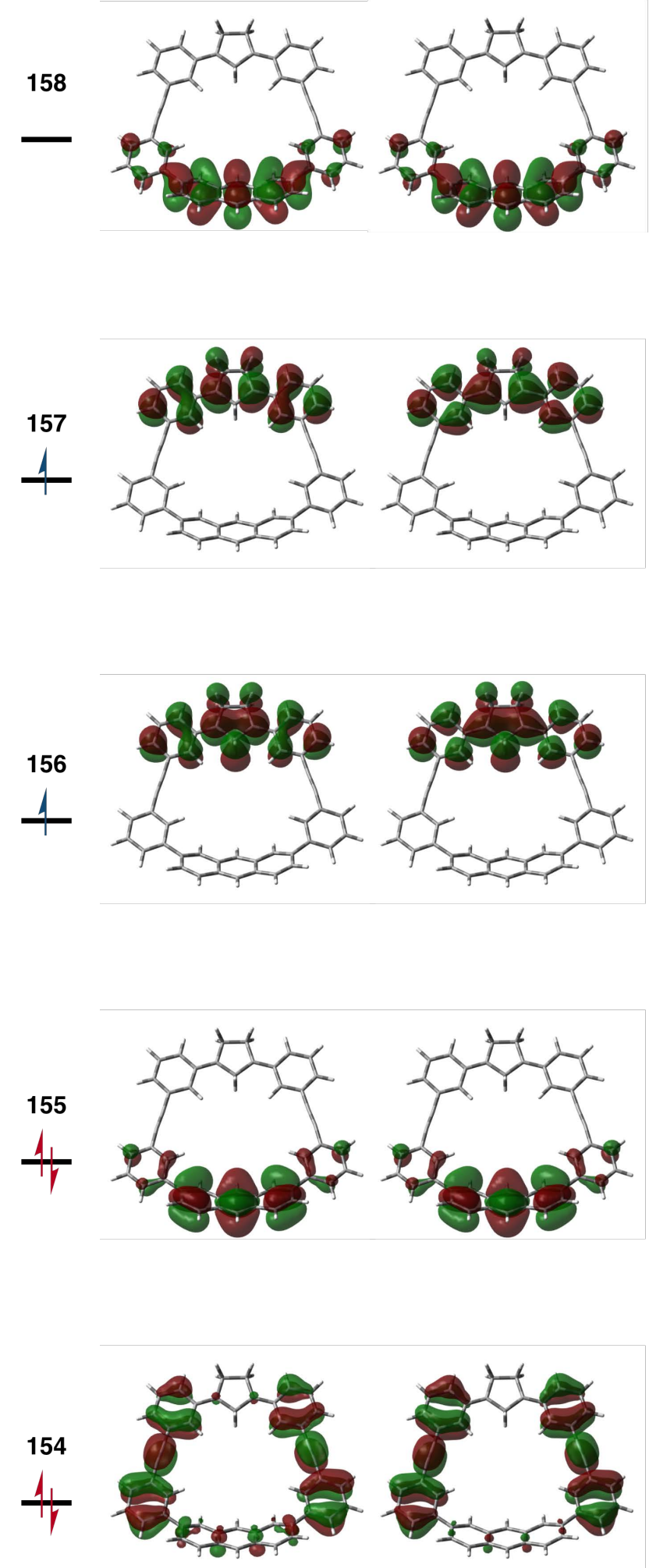

(b)
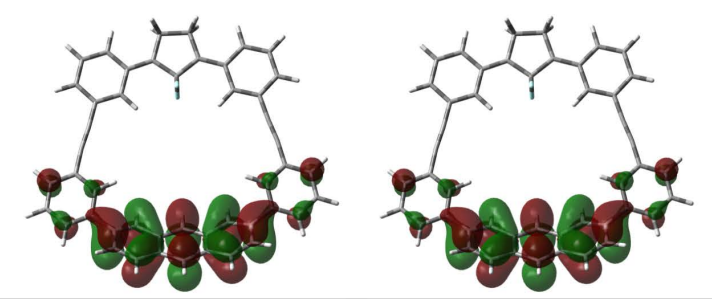

165

1
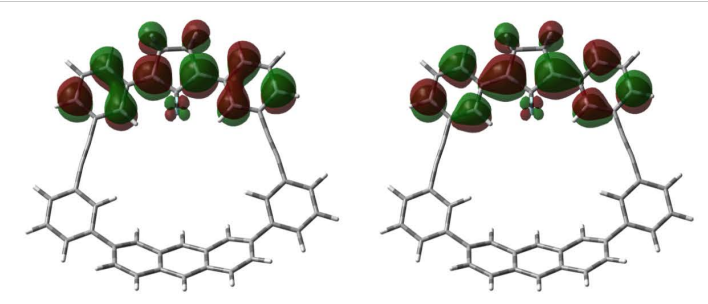

164
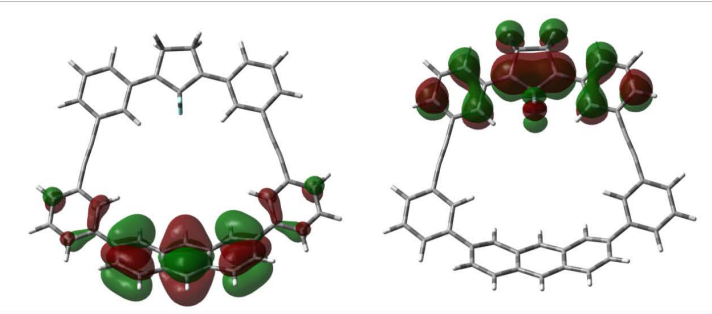

163
4
4
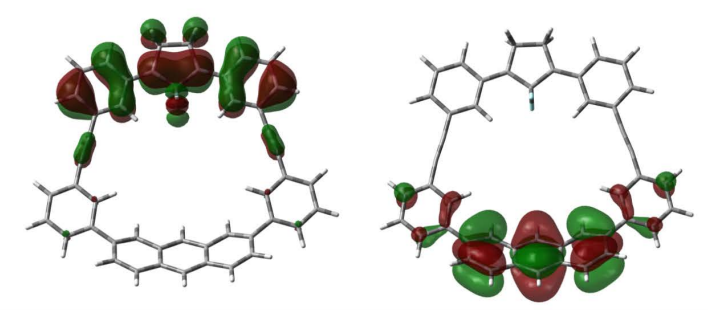

162
4
4

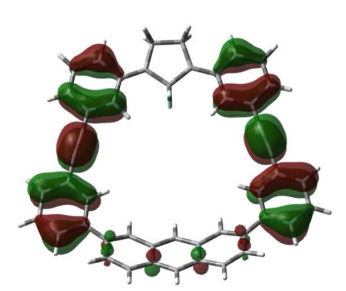

Figure S3. Selected molecular orbitals of (a) DR3H1 and (b) DR3F1. 


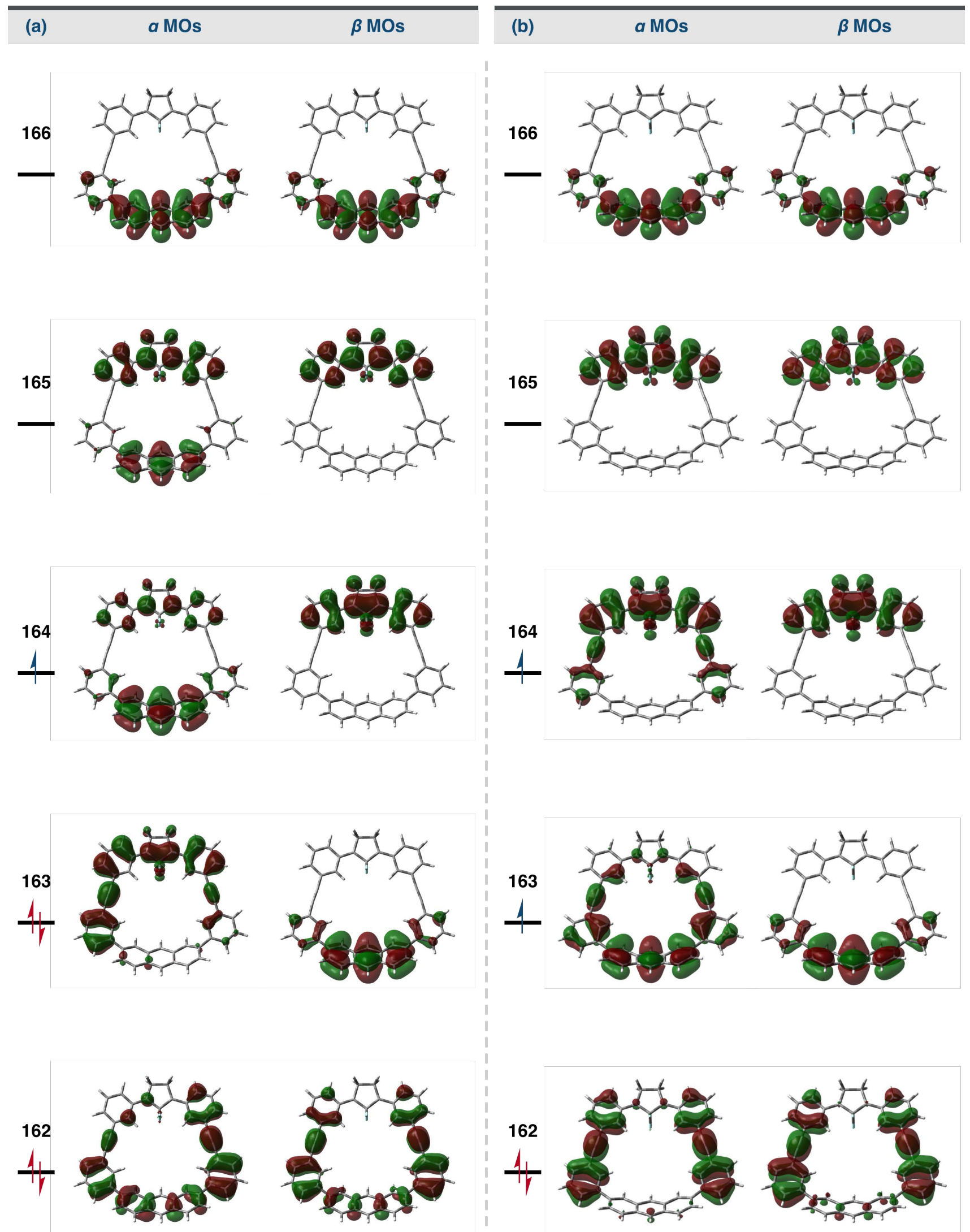

Figure S4. Selected molecular orbitals of (a) DR3F1 ${ }^{+}$and (b) DR3F1 ${ }^{2+}$. 


\begin{tabular}{lll}
\hline (a) & $a \mathrm{MOs}$ & $\beta \mathrm{MOs}$
\end{tabular}

(b)
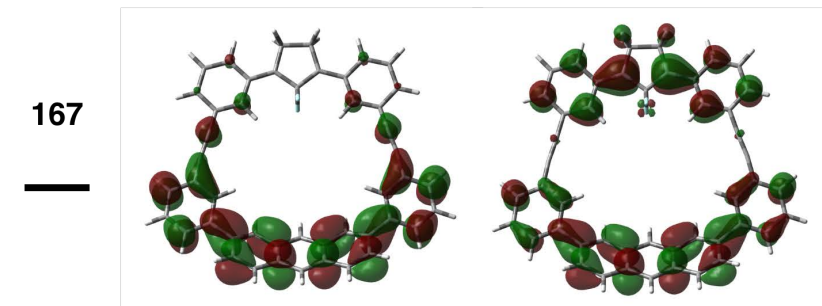

166
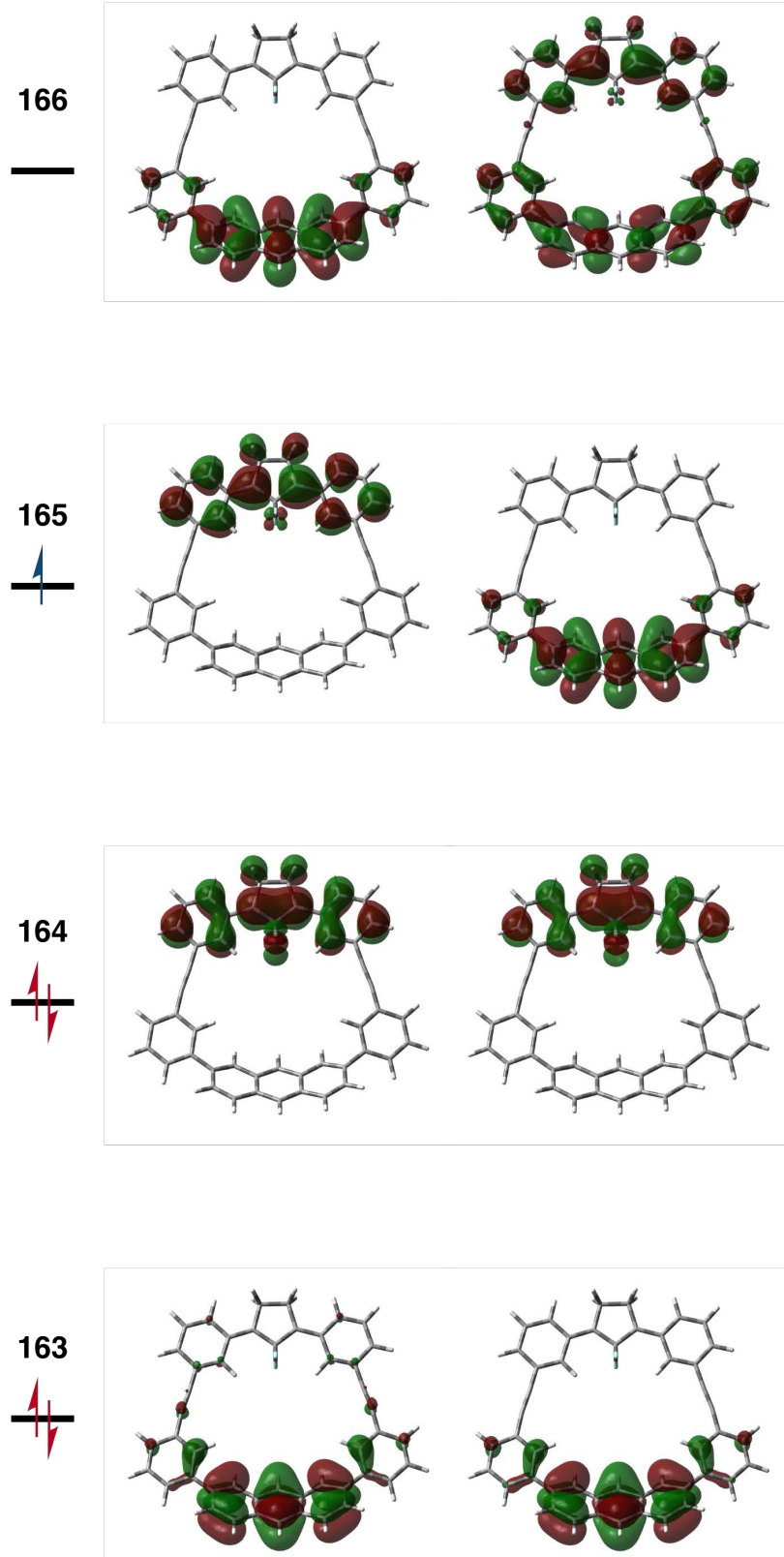
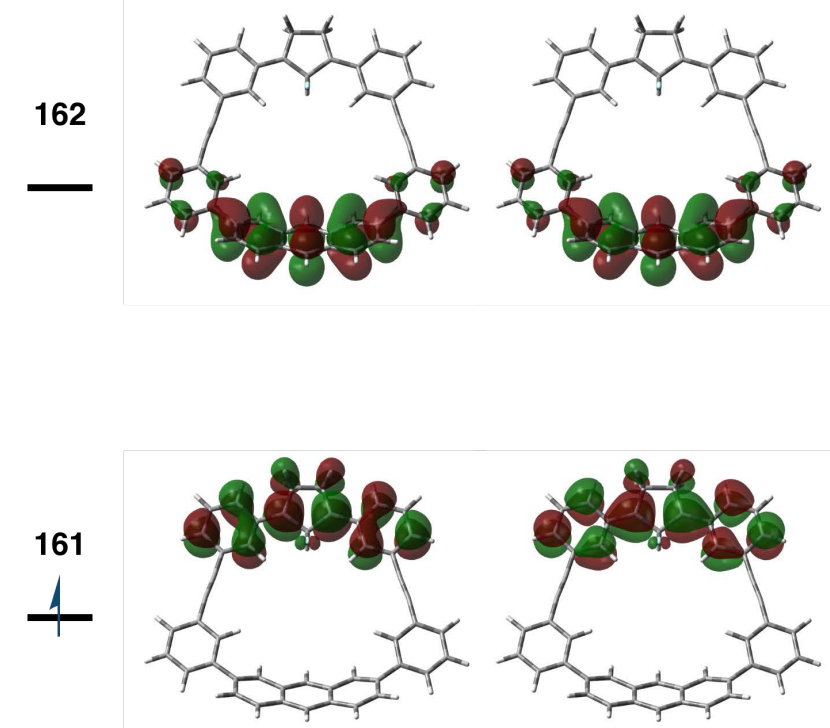

160

1
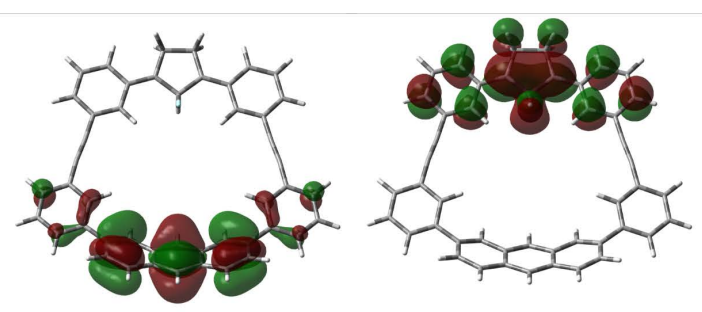

$\frac{159}{4}$
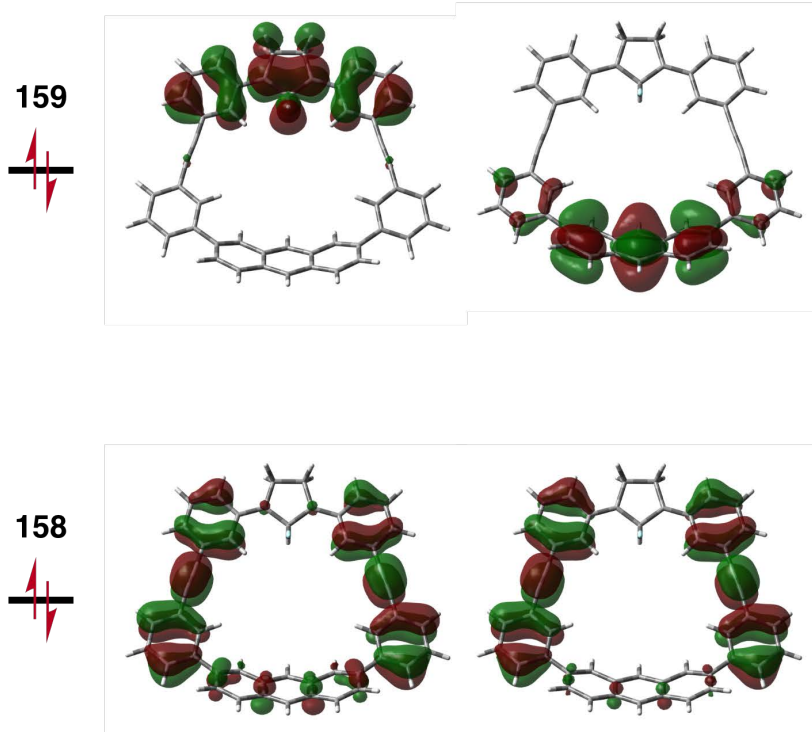

Figure S5. Selected molecular orbitals of (a) DR3F1 and (b) DR3HF1. 


(a) $\quad$ a MOs $\quad \beta$ MOs
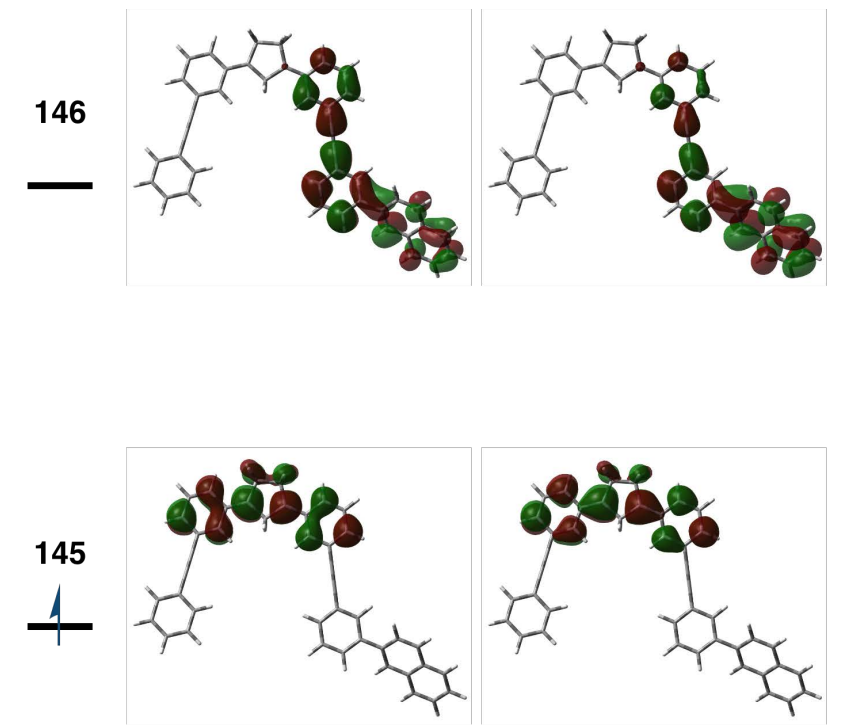

144
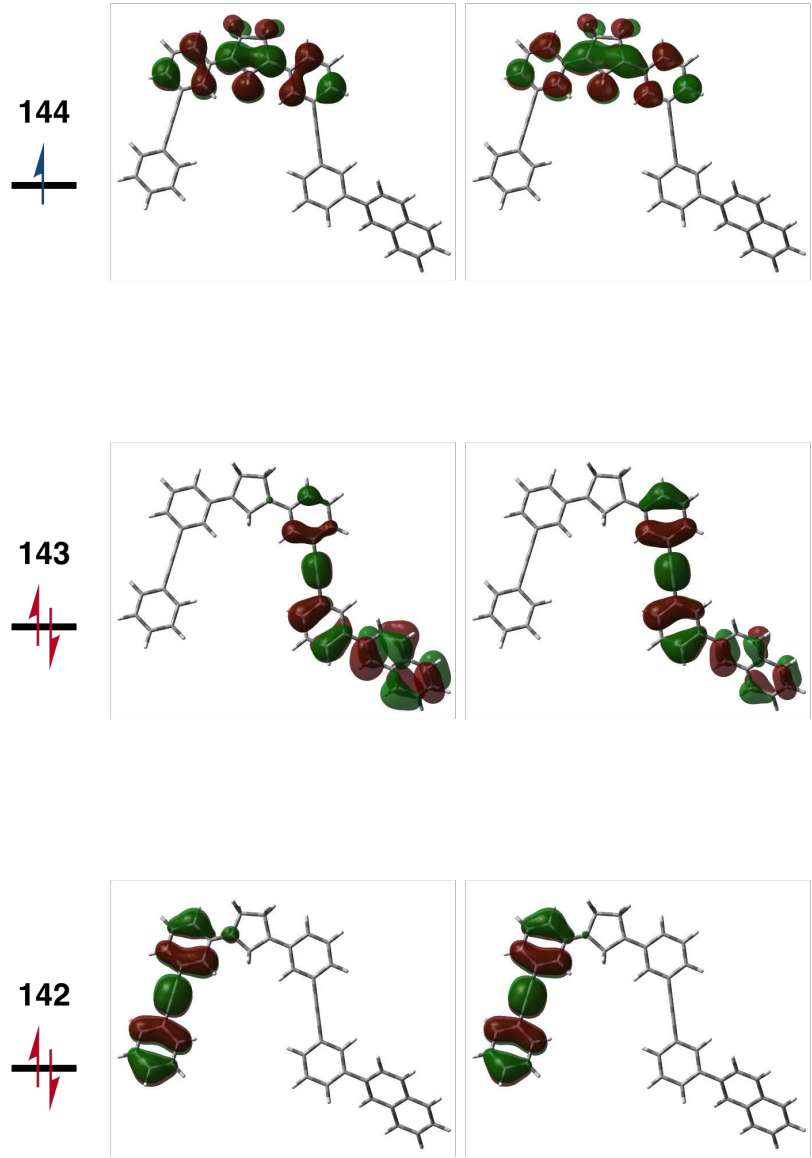

(b)

a MOs

$\beta$ MOs

154
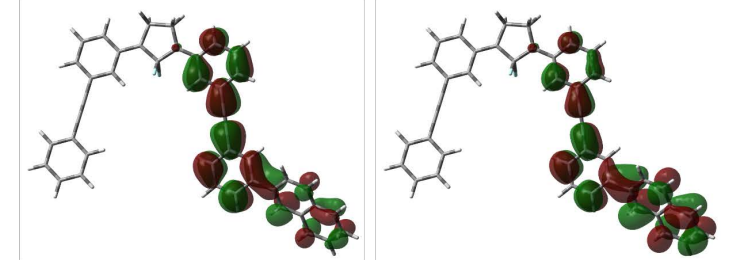

\begin{tabular}{l}
153 \\
-1 \\
\hline
\end{tabular}
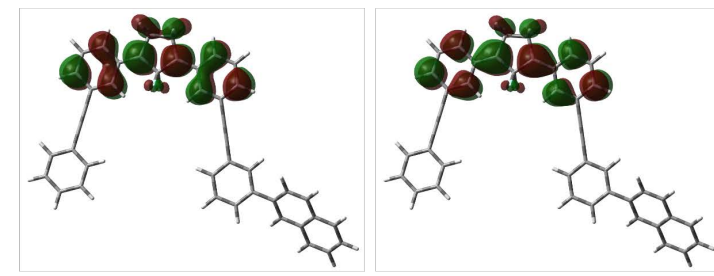

151

$\frac{4}{1}$
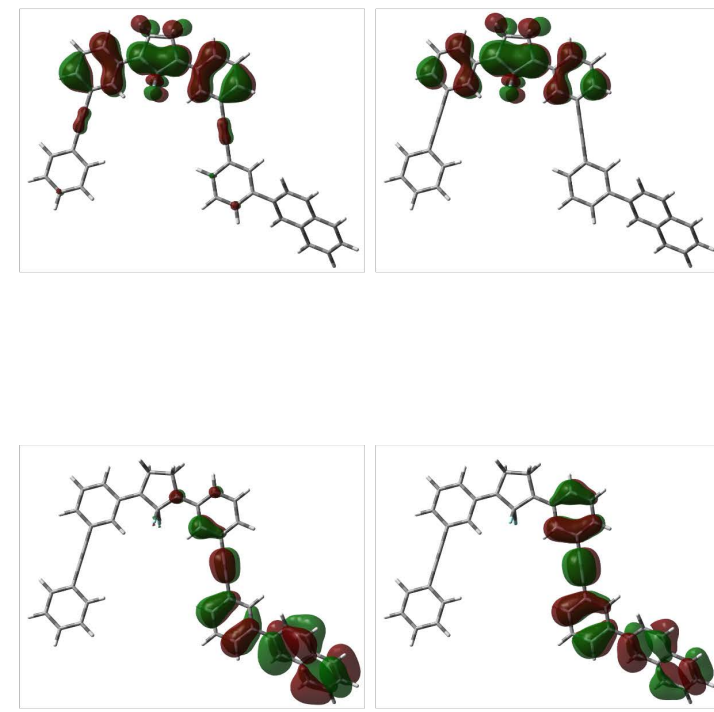

\begin{tabular}{c}
152 \\
$\perp$ \\
\hline
\end{tabular}
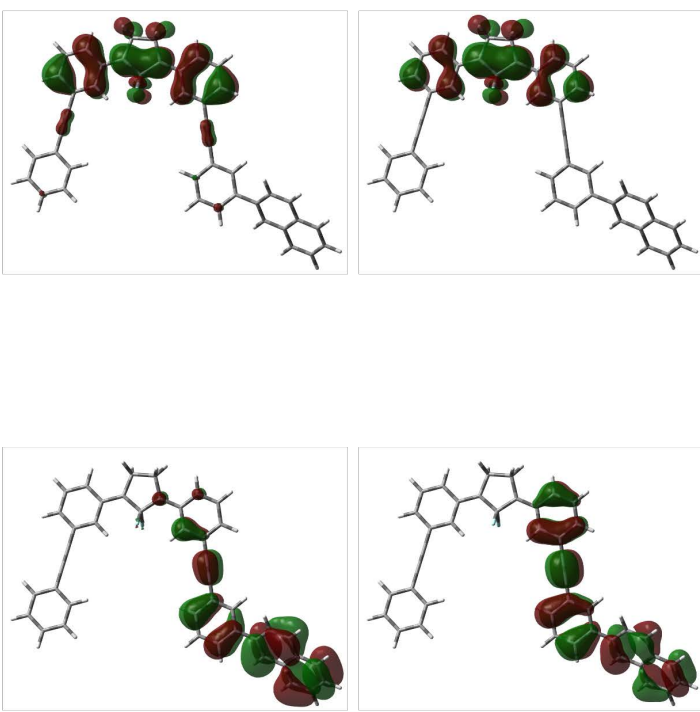

150

4
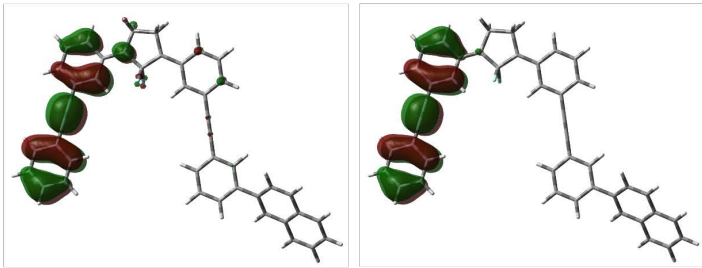

Figure S6. Selected molecular orbitals of (a) DR4H0 and (b) DR4F0. 


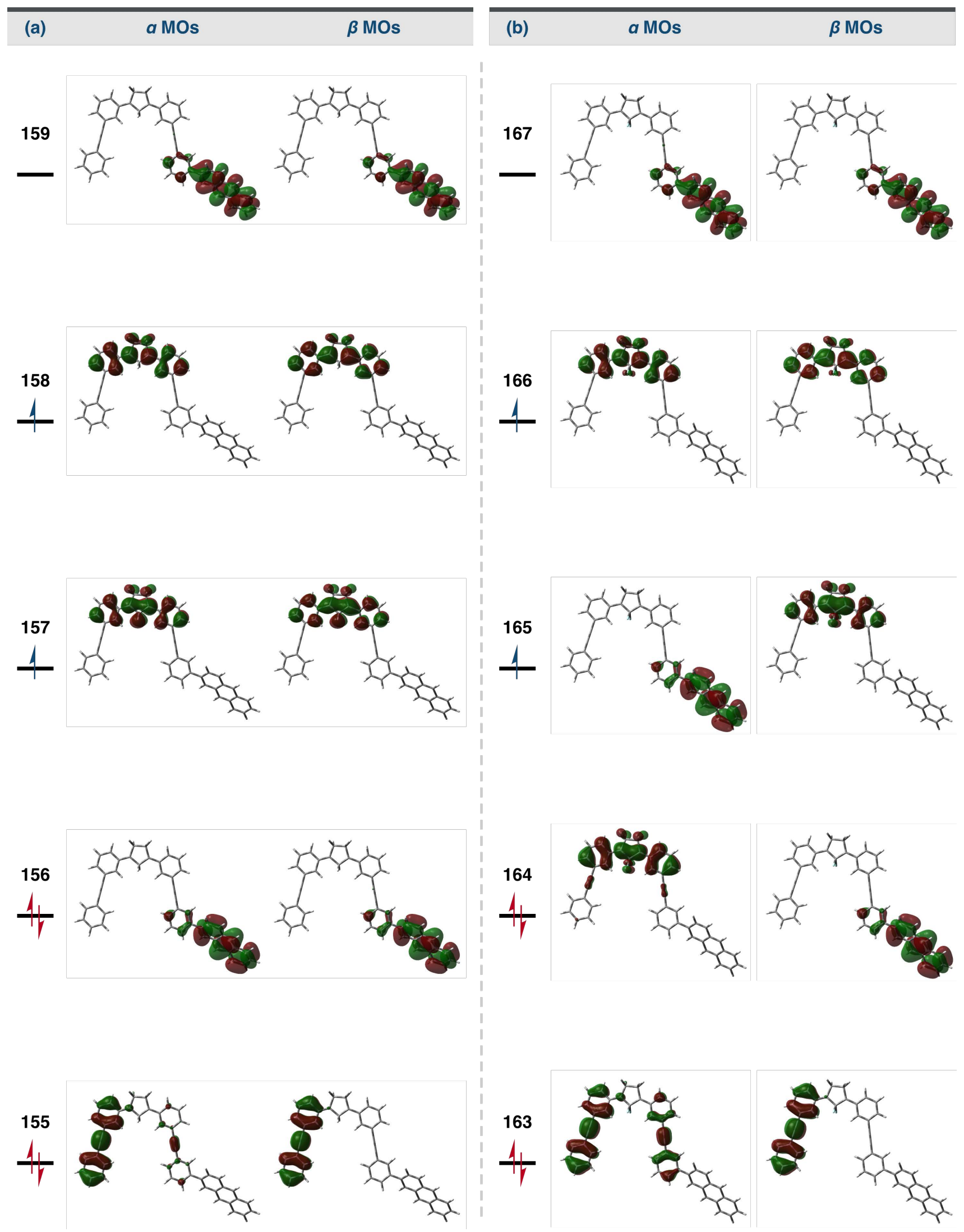

Figure S7. Selected molecular orbitals of (a) DR4H1 and (b) DR4F1. 

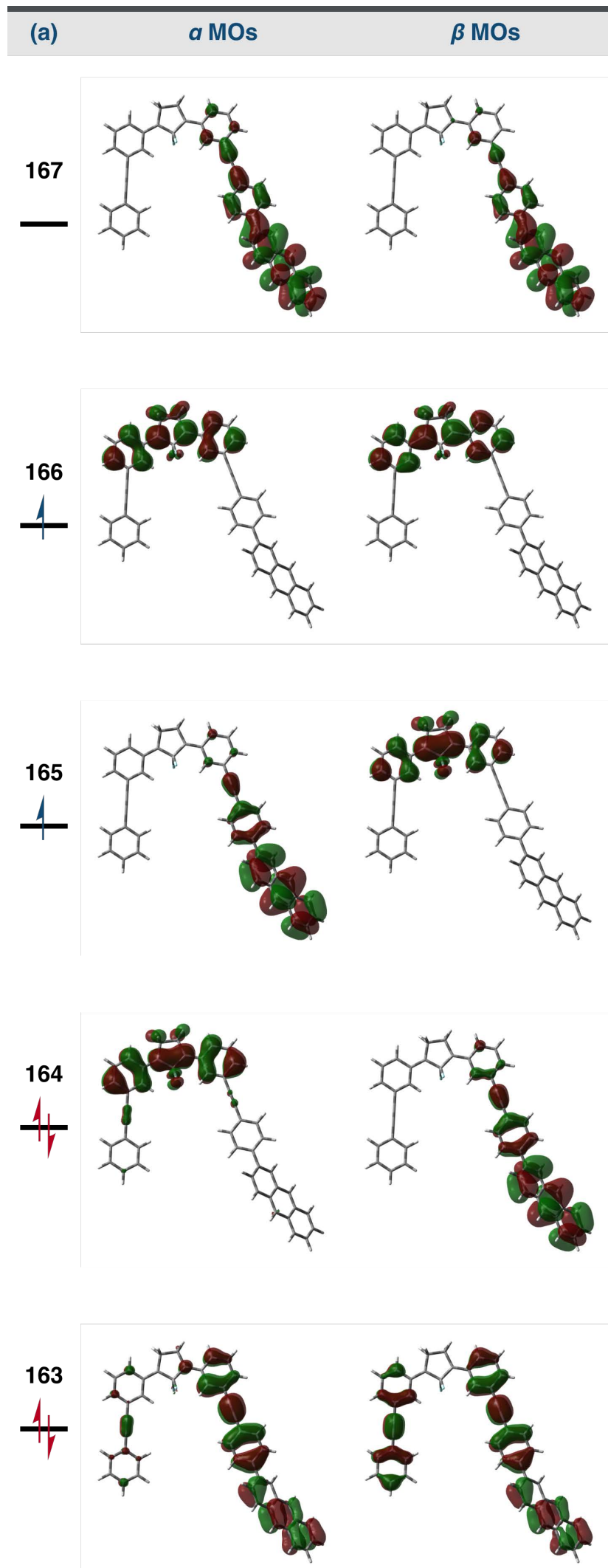

(b)

a MOs

$\beta$ MOs

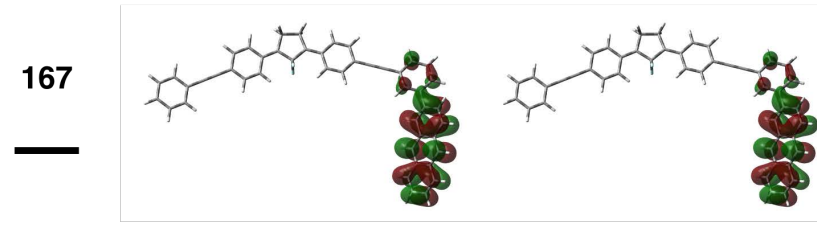

166

4
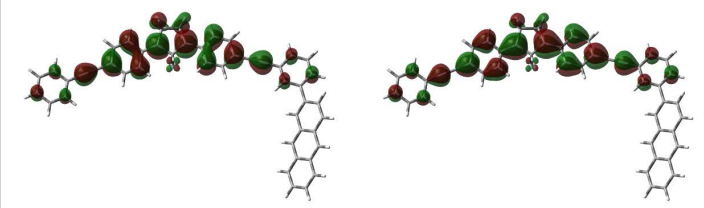

165

4

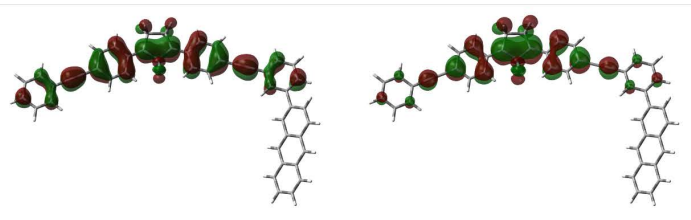

164
4
41
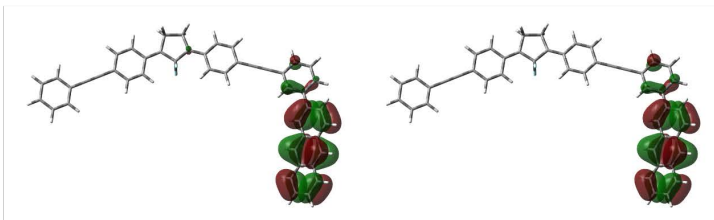

163

$\frac{1}{4}$

Figure S8. Selected molecular orbitals of (a) DR5a and (b) DR5b. 


\section{Spin Density Maps}

Spin densities of DR1-5 are visualized with an iso-surface value of 0.0015 a.u. using Gauss View 6.0.16 program.

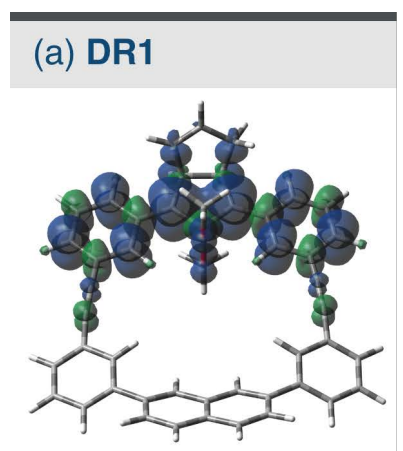

(b) DR2

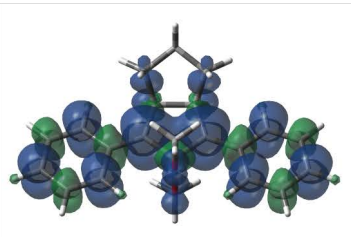

(e) DR3H1

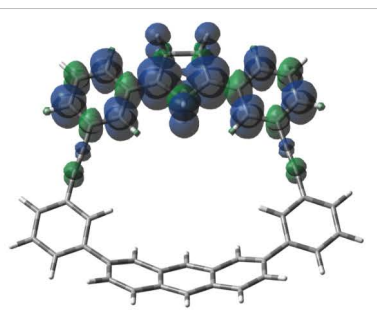

(f) DR3F1

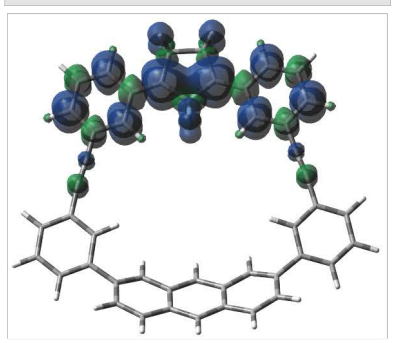

(i) DR3F1

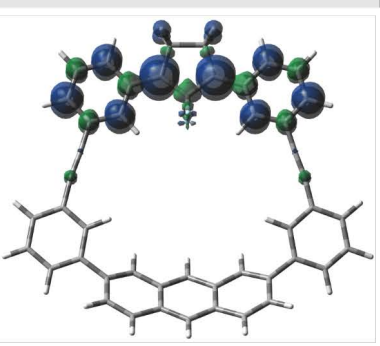

(m) DR4H1

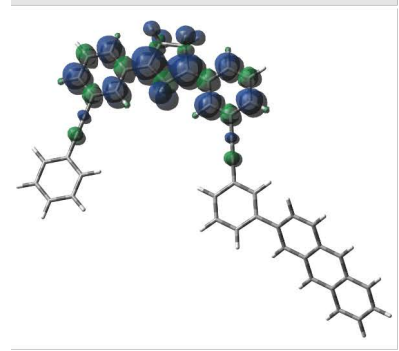

(j) DR3HF1

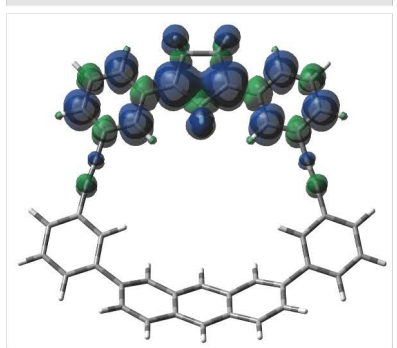

(n) DR4F1

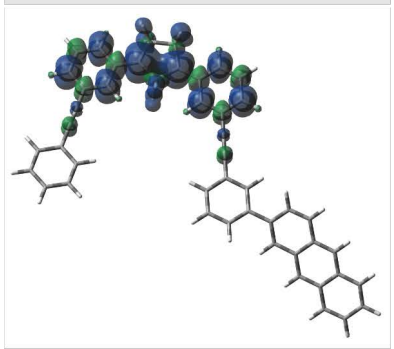

(c) DR3HO

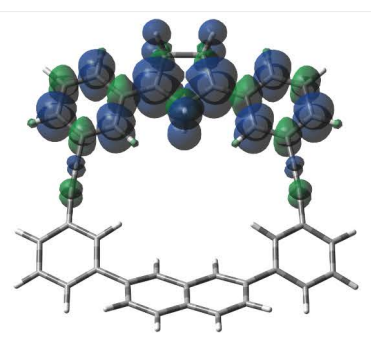

(g) DR3F1+

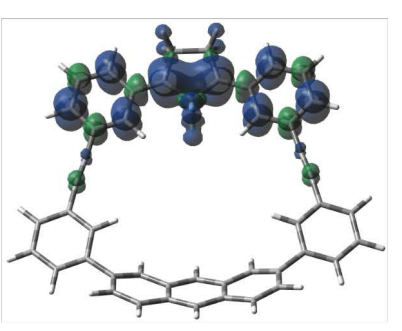

(k) DR4HO

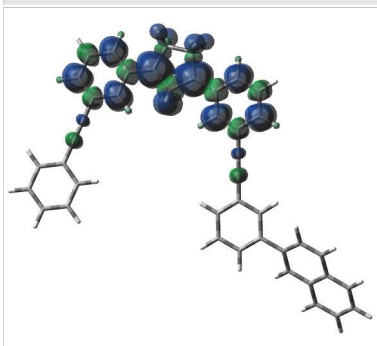

(o) DR5a

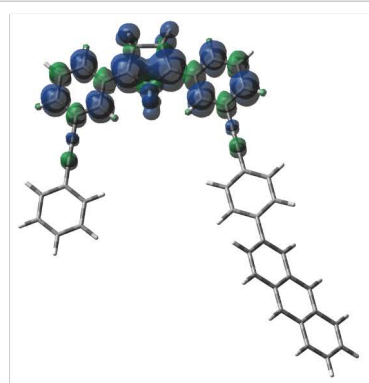

(d) DR3F0

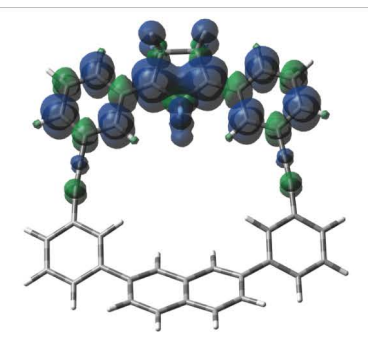

(h) DR3F1 ${ }^{2+}$

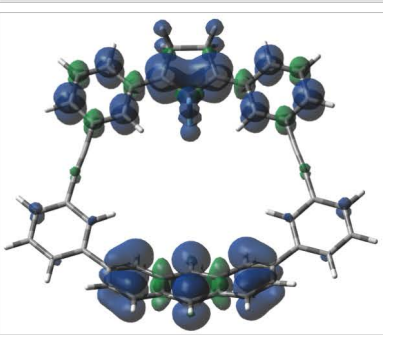

(I) DR4F0

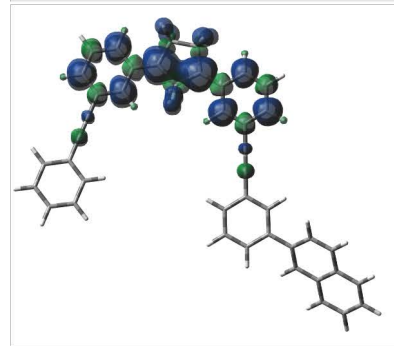

(p) DR5b

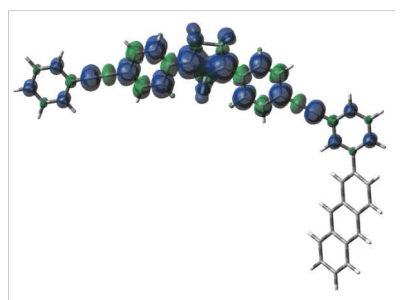

Figure S9. Spin density maps of (a) DR1, (b) DR2, (c) DR3H0, (d) DR3F0, (e) DR3H1, (f) DR3F1, (g) DR3F1 ${ }^{+}$, (h) DR3F1 ${ }^{2+}$, (i) DR3F1-, (j) DR3HF1, (k) DR4H0, (l) DR4F0, (m) DR4H1 (n) DR4F1, (o) DR5a and (p) DR5b. 


\section{Cartesian Coordinates of Geometries}

Quantum chemical computations in gas phase have been performed with the Gaussian 16 (Revision B.01) suite of programs. Charge, spin multiplicity, number of imaginary frequencies, energies (in Hartree) and Cartesian coordinates (in $\AA$ ) of computed geometries at UB3LYP, UwB97X-D and UM06-2X functionals with 6-31G(d) (for DR3F1' : 6-31+G(d)) basis set are listed in this section.

Table S1. List of calculated geometries.

\begin{tabular}{cccc}
\hline & B3LYP & $\omega$ B97X-D & M06-2X \\
\hline DR1 & S12 & - & - \\
DR2 & S14 & - & - \\
DR3H0 & S15 & S17 & S19 \\
DR3F0 & S21 & S23 & S25 \\
DR3H1 & S27 & S29 & S31 \\
DR3F1 & S33 & S35 & S37 \\
DR3F1 & S39 & - & - \\
DR3F1 ${ }^{2+}$ & S41 & - & - \\
DR3F1 & S47 & - & - \\
DR3HF1 & S49 & - & - \\
DR4H0 & S51 & S53 & S55 \\
DR4F0 & S57 & S59 & S61 \\
DR4H1 & S63 & S65 & S67 \\
DR4F1 & S69 & S71 & S73 \\
DR5a & S75 & - & - \\
DR5b & S 77 & - & - \\
\hline
\end{tabular}




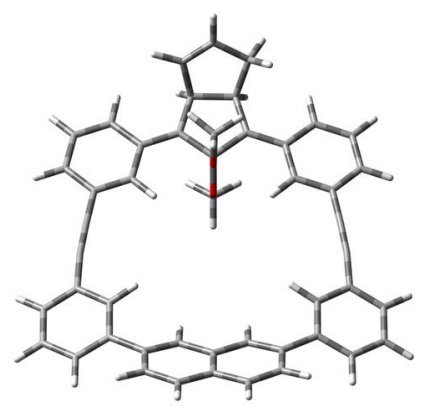

\#p opt freq ub3lyp/6-31g(d)

Charge $=0$, Multiplicity $=3$, Point group $=C_{1}$

Number of Imaginary Frequencies $=0$

Sum of electronic and zero-point Energies $=-1999.175070$ Hartree

Sum of electronic and thermal Energies = -1999.134830 Hartree

Sum of electronic and enthalpy Energies $=-1999.133886$ Hartree

Sum of electronic and thermal Free Energies $=-1999.248558$ Hartree

\begin{tabular}{|c|c|c|c|c|c|c|c|}
\hline \multirow[b]{2}{*}{ Atom } & \multicolumn{3}{|c|}{ Cartesian Coordinates } & \multirow[b]{2}{*}{ Atom } & \multicolumn{3}{|c|}{ Cartesian Coordinates } \\
\hline & $X$ & $\boldsymbol{Y}$ & $Z$ & & $X$ & $\boldsymbol{Y}$ & $Z$ \\
\hline $\mathrm{C}$ & -4.172426 & -1.267386 & -0.058286 & $\mathrm{C}$ & 2.808005 & -6.032020 & -0.648785 \\
\hline $\mathrm{C}$ & -3.261966 & -0.046856 & -0.101715 & $\mathrm{C}$ & 4.201145 & -6.034621 & -0.666559 \\
\hline $\mathrm{C}$ & -4.211230 & 1.146410 & -0.071045 & $\mathrm{C}$ & 4.259082 & -3.665934 & -0.113672 \\
\hline $\mathrm{C}$ & -5.651530 & 0.709706 & -0.012902 & $\mathrm{H}$ & 2.317094 & -2.776146 & 0.181811 \\
\hline $\mathrm{C}$ & -5.619631 & -0.857360 & 0.062447 & $\mathrm{H}$ & 2.249185 & -6.938481 & -0.859347 \\
\hline $\mathrm{C}$ & -3.790495 & 2.505598 & -0.192140 & $\mathrm{H}$ & 4.732447 & -6.953898 & -0.898151 \\
\hline $\mathrm{C}$ & -2.982382 & 5.219581 & -0.449507 & $\mathrm{H}$ & 6.009115 & -4.887335 & -0.458512 \\
\hline $\mathrm{C}$ & -4.740972 & 3.559647 & -0.327824 & $\mathrm{C}$ & 4.981919 & -2.396545 & 0.155603 \\
\hline $\mathrm{C}$ & -2.414818 & 2.867539 & -0.192623 & $\mathrm{H}$ & 3.489071 & -1.173694 & -0.779070 \\
\hline $\mathrm{C}$ & -2.009998 & 4.203508 & -0.318909 & $\mathrm{C}$ & 4.422347 & -1.188774 & -0.223312 \\
\hline $\mathrm{C}$ & -4.336324 & 4.882106 & -0.454000 & $\mathrm{C}$ & 6.857103 & -1.183446 & 1.144951 \\
\hline $\mathrm{H}$ & -5.800633 & 3.327410 & -0.345695 & $\mathrm{C}$ & 5.021769 & 0.050909 & 0.104135 \\
\hline $\mathrm{H}$ & -1.664502 & 2.094638 & -0.092132 & $\mathrm{C}$ & 6.234202 & -2.372496 & 0.838131 \\
\hline $\mathrm{H}$ & -5.083935 & 5.663848 & -0.560077 & $\mathrm{C}$ & 6.265996 & 0.063750 & 0.807681 \\
\hline $\mathrm{H}$ & -2.666522 & 6.253247 & -0.546897 & $\mathrm{C}$ & 4.391055 & 1.277668 & -0.212998 \\
\hline $\mathrm{C}$ & -3.721868 & -2.616901 & -0.156057 & $\mathrm{H}$ & 6.684597 & -3.310147 & 1.151473 \\
\hline $\mathrm{C}$ & -2.853460 & -5.316662 & -0.362416 & $\mathrm{H}$ & 7.770257 & 1.347444 & 1.693269 \\
\hline $\mathrm{C}$ & -2.337800 & -2.945013 & -0.188432 & $\mathrm{H}$ & 7.803395 & -1.188648 & 1.681100 \\
\hline $\mathrm{C}$ & -4.649180 & -3.697667 & -0.231801 & $\mathrm{C}$ & 4.919401 & 2.496086 & 0.176584 \\
\hline $\mathrm{C}$ & -4.215204 & -5.012665 & -0.333922 & $\mathrm{H}$ & 3.458970 & 1.243365 & -0.769862 \\
\hline $\mathrm{C}$ & -1.903607 & -4.273749 & -0.288596 & $\mathrm{C}$ & 6.171384 & 2.498017 & 0.860123 \\
\hline $\mathrm{H}$ & -1.604746 & -2.151610 & -0.129949 & $\mathrm{H}$ & 6.597479 & 3.444003 & 1.182241 \\
\hline $\mathrm{H}$ & -5.714661 & -3.492439 & -0.221329 & $\mathrm{C}$ & 6.824583 & 1.322686 & 1.156577 \\
\hline $\mathrm{H}$ & -4.945620 & -5.815271 & -0.394022 & $\mathrm{O}$ & -2.402316 & -0.040098 & -1.243283 \\
\hline $\mathrm{H}$ & -2.514623 & -6.344690 & -0.440044 & $\mathrm{O}$ & -2.275171 & -0.029092 & 0.930207 \\
\hline $\mathrm{C}$ & -0.504924 & -4.555333 & -0.314061 & $\mathrm{C}$ & -3.037049 & -0.056462 & -2.511797 \\
\hline $\mathrm{C}$ & 0.694635 & -4.755524 & -0.337275 & $\mathrm{H}$ & -3.645986 & -0.959193 & -2.656015 \\
\hline
\end{tabular}




\begin{tabular}{cccccccc}
\hline & \multicolumn{3}{c}{ Cartesian Coordinates } & \multicolumn{4}{c}{ Cartesian Coordinates } \\
Atom & $\boldsymbol{X}$ & $\boldsymbol{Y}$ & $\boldsymbol{Z}$ & Atom & $\boldsymbol{X}$ & $\boldsymbol{Y}$ & $\boldsymbol{Z}$ \\
\hline $\mathrm{C}$ & -0.618438 & 4.519106 & -0.318851 & $\mathrm{H}$ & -3.664298 & 0.831474 & -2.669503 \\
$\mathrm{C}$ & 0.575952 & 4.749268 & -0.325063 & $\mathrm{H}$ & -2.229523 & -0.053321 & -3.247352 \\
$\mathrm{C}$ & 1.997015 & 4.870575 & -0.331339 & $\mathrm{C}$ & -2.756994 & 0.009676 & 2.265002 \\
$\mathrm{C}$ & 4.801338 & 4.970988 & -0.363782 & $\mathrm{H}$ & -3.311196 & 0.934963 & 2.472589 \\
$\mathrm{C}$ & 2.657839 & 6.081991 & -0.609075 & $\mathrm{H}$ & -1.869401 & -0.023959 & 2.900754 \\
$\mathrm{C}$ & 2.765323 & 3.725460 & -0.058965 & $\mathrm{H}$ & -3.399622 & -0.849273 & 2.496342 \\
$\mathrm{C}$ & 4.165548 & 3.749089 & -0.084376 & $\mathrm{H}$ & -6.181953 & 1.038857 & -0.917548 \\
$\mathrm{C}$ & 4.050500 & 6.120221 & -0.617990 & $\mathrm{H}$ & -6.220114 & -1.311723 & -0.741916 \\
$\mathrm{H}$ & 2.077322 & 6.975515 & -0.816196 & $\mathrm{C}$ & -6.246554 & -1.164243 & 1.414718 \\
$\mathrm{H}$ & 2.244995 & 2.808004 & 0.192396 & $\mathrm{H}$ & -6.308651 & -2.176349 & 1.803212 \\
$\mathrm{H}$ & 4.559610 & 7.054591 & -0.838767 & $\mathrm{C}$ & -6.678966 & -0.069347 & 2.040779 \\
$\mathrm{H}$ & 5.885751 & 5.017452 & -0.409350 & $\mathrm{H}$ & -7.158092 & -0.062014 & 3.016772 \\
$\mathrm{C}$ & 2.118271 & -4.840226 & -0.356978 & $\mathrm{C}$ & -6.448898 & 1.192755 & 1.250907 \\
$\mathrm{C}$ & 4.924122 & -4.868859 & -0.406857 & $\mathrm{H}$ & -5.89103 & 1.943774 & 1.823953 \\
$\mathrm{C}$ & 2.858906 & -3.677983 & -0.080480 & $\mathrm{H}$ & -7.400658 & 1.665513 & 0.968924 \\
\hline
\end{tabular}




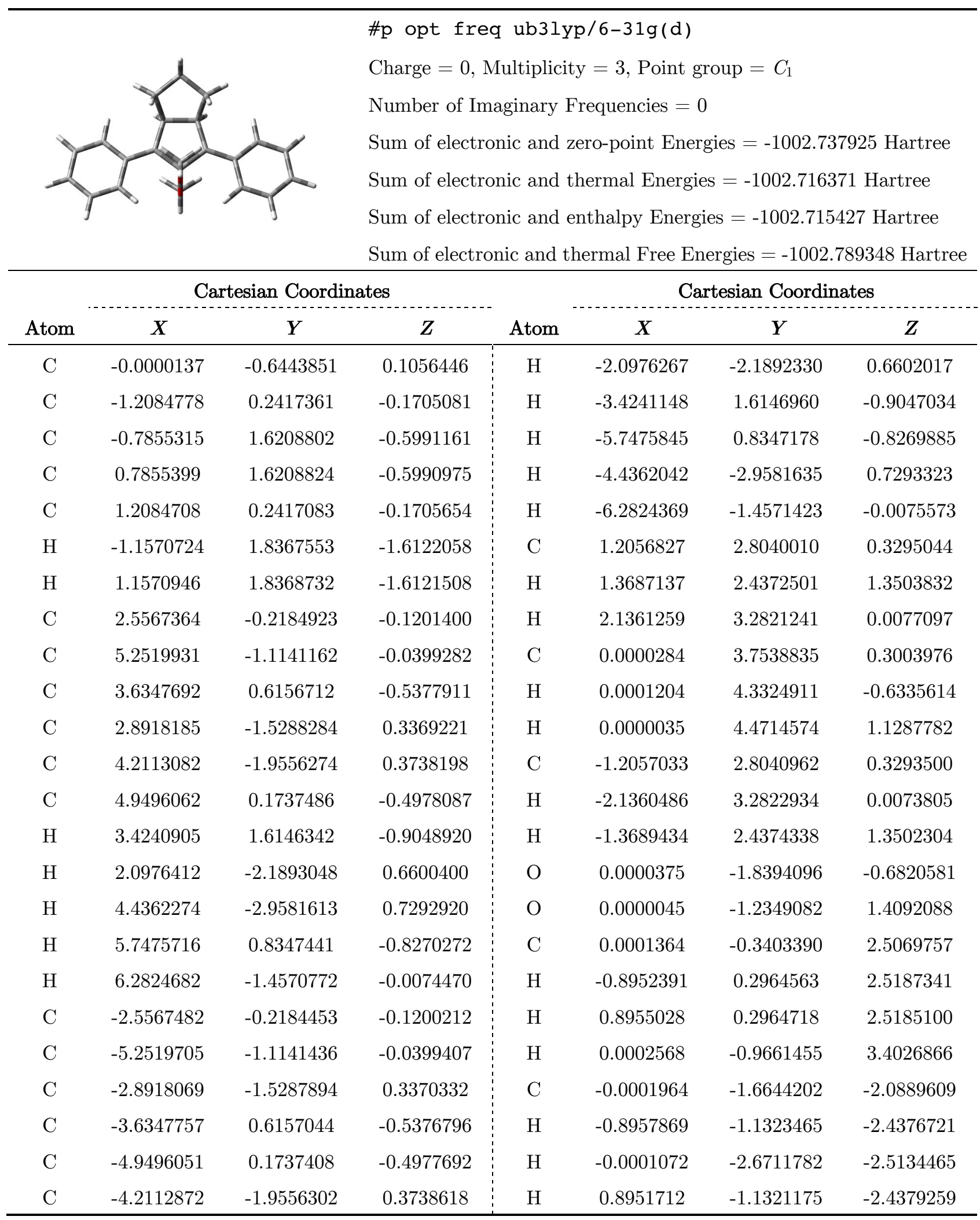




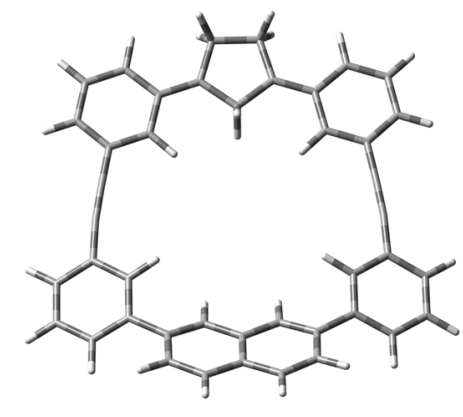

\#p opt freq ub3lyp/6-31g(d)

Charge $=0$, Multiplicity $=3$, Point group $=C_{\mathrm{S}}$

Number of Imaginary Frequencies $=1, v_{i}=-21.72$

Sum of electronic and zero-point Energies $=-1654.746713$ Hartree

Sum of electronic and thermal Energies $=-1654.714424$ Hartree

Sum of electronic and enthalpy Energies = -1654.713480 Hartree

Sum of electronic and thermal Free Energies $=-1654.810890$ Hartree

\begin{tabular}{|c|c|c|c|c|c|c|c|}
\hline \multirow[b]{2}{*}{ Atom } & \multicolumn{3}{|c|}{ Cartesian Coordinates } & \multicolumn{4}{|c|}{ Cartesian Coordinates } \\
\hline & $X$ & $Y$ & $Z$ & Atom & $X$ & $Y$ & $Z$ \\
\hline $\mathrm{C}$ & -4.960944 & -1.199051 & 0.152473 & $\mathrm{H}$ & 1.557643 & 2.774564 & 0.224479 \\
\hline $\mathrm{C}$ & -4.049730 & -0.000029 & 0.110383 & $\mathrm{H}$ & 3.832868 & 7.008671 & -0.933158 \\
\hline $\mathrm{C}$ & -4.960967 & 1.198978 & 0.152417 & $\mathrm{H}$ & 5.170151 & 4.963406 & -0.581594 \\
\hline $\mathrm{C}$ & -6.405949 & 0.774620 & 0.239463 & $\mathrm{C}$ & 1.290524 & -4.838883 & -0.282995 \\
\hline $\mathrm{C}$ & -6.405925 & -0.774718 & 0.239643 & $\mathrm{C}$ & 4.089830 & -4.923010 & -0.475335 \\
\hline $\mathrm{C}$ & -4.526954 & 2.552954 & 0.108924 & $\mathrm{C}$ & 2.066957 & -3.689401 & -0.057195 \\
\hline $\mathrm{C}$ & -3.672626 & 5.264206 & 0.006929 & $\mathrm{C}$ & 1.941899 & -6.046737 & -0.596622 \\
\hline $\mathrm{C}$ & -5.458079 & 3.631500 & 0.142824 & $\mathrm{C}$ & 3.332045 & -6.076747 & -0.685075 \\
\hline $\mathrm{C}$ & -3.149906 & 2.891107 & 0.025989 & $\mathrm{C}$ & 3.463585 & -3.704641 & -0.161972 \\
\hline $\mathrm{C}$ & -2.718109 & 4.223803 & -0.025744 & $\mathrm{H}$ & 1.557690 & -2.774536 & 0.224385 \\
\hline $\mathrm{C}$ & -5.030658 & 4.951362 & 0.092141 & $\mathrm{H}$ & 1.355999 & -6.944118 & -0.768709 \\
\hline $\mathrm{H}$ & -6.520200 & 3.417686 & 0.207987 & $\mathrm{H}$ & 3.832978 & -7.008611 & -0.933247 \\
\hline $\mathrm{H}$ & -2.402642 & 2.105232 & -0.000997 & $\mathrm{H}$ & 5.170234 & -4.963336 & -0.581636 \\
\hline $\mathrm{H}$ & -5.763730 & 5.753404 & 0.118780 & $\mathrm{C}$ & 4.221108 & -2.446068 & 0.057668 \\
\hline $\mathrm{H}$ & -3.340097 & 6.296242 & -0.033047 & $\mathrm{H}$ & 2.707019 & -1.208763 & -0.821163 \\
\hline $\mathrm{C}$ & -4.526907 & -2.553021 & 0.108993 & $\mathrm{C}$ & 3.663706 & -1.233052 & -0.306999 \\
\hline $\mathrm{C}$ & -3.672530 & -5.264258 & 0.007028 & $\mathrm{C}$ & 6.157372 & -1.253425 & 0.949273 \\
\hline $\mathrm{C}$ & -3.149858 & -2.891148 & 0.025972 & $\mathrm{C}$ & 4.293880 & 0.000029 & -0.015349 \\
\hline $\mathrm{C}$ & -5.458007 & -3.631584 & 0.142999 & $\mathrm{C}$ & 5.503944 & -2.435574 & 0.681177 \\
\hline $\mathrm{C}$ & -5.030562 & -4.951440 & 0.092328 & $\mathrm{C}$ & 5.568461 & 0.000034 & 0.631502 \\
\hline $\mathrm{C}$ & -2.718036 & -4.223837 & -0.025746 & $\mathrm{C}$ & 3.663684 & 1.233103 & -0.306977 \\
\hline $\mathrm{H}$ & -2.402613 & -2.105258 & -0.001097 & $\mathrm{H}$ & 5.954585 & -3.377384 & 0.981358 \\
\hline $\mathrm{H}$ & -6.520127 & -3.417791 & 0.208238 & $\mathrm{H}$ & 7.127132 & 1.268833 & 1.441378 \\
\hline $\mathrm{H}$ & -5.763616 & -5.753496 & 0.119047 & $\mathrm{H}$ & 7.127152 & -1.268750 & 1.441362 \\
\hline $\mathrm{H}$ & -3.339983 & -6.296289 & -0.032939 & $\mathrm{C}$ & 4.221066 & 2.446124 & 0.057706 \\
\hline $\mathrm{C}$ & -1.323436 & -4.512865 & -0.112800 & $\mathrm{H}$ & 2.706997 & 1.208804 & -0.821143 \\
\hline $\mathrm{C}$ & -0.128625 & -4.727388 & -0.192491 & $\mathrm{C}$ & 5.503905 & 2.435641 & 0.681211 \\
\hline
\end{tabular}




\begin{tabular}{cccccccc}
\hline & \multicolumn{3}{c}{ Cartesian Coordinates } & \multicolumn{4}{c}{ Cartesian Coordinates } \\
Atom & $\boldsymbol{X}$ & \multicolumn{1}{c}{$\boldsymbol{Y}$} & $\boldsymbol{Z}$ & Atom & $\boldsymbol{X}$ & $\boldsymbol{Y}$ & $\boldsymbol{Z}$ \\
\hline $\mathrm{C}$ & -1.323508 & 4.512859 & -0.112708 & $\mathrm{H}$ & 5.954531 & 3.377454 & 0.981406 \\
$\mathrm{C}$ & -0.128697 & 4.727406 & -0.192337 & $\mathrm{C}$ & 6.157352 & 1.253499 & 0.949289 \\
$\mathrm{C}$ & 1.290448 & 4.838911 & -0.282882 & $\mathrm{H}$ & -6.991586 & 1.172854 & -0.602384 \\
$\mathrm{C}$ & 4.089750 & 4.923068 & -0.475275 & $\mathrm{H}$ & -6.991702 & -1.173176 & -0.601997 \\
$\mathrm{C}$ & 1.941805 & 6.046775 & -0.596509 & $\mathrm{H}$ & -6.886764 & 1.173495 & 1.145170 \\
$\mathrm{C}$ & 2.066896 & 3.689434 & -0.057108 & $\mathrm{H}$ & -6.886564 & -1.173387 & 1.145537 \\
$\mathrm{C}$ & 3.463523 & 3.704689 & -0.161913 & $\mathrm{H}$ & -3.331261 & -0.000003 & 0.952289 \\
$\mathrm{C}$ & 3.331949 & 6.076799 & -0.684989 & $\mathrm{H}$ & -3.412064 & -0.000043 & -0.793606 \\
$\mathrm{H}$ & 1.355893 & 6.944152 & -0.768572 & & & & \\
\hline
\end{tabular}




\section{DR3H0}

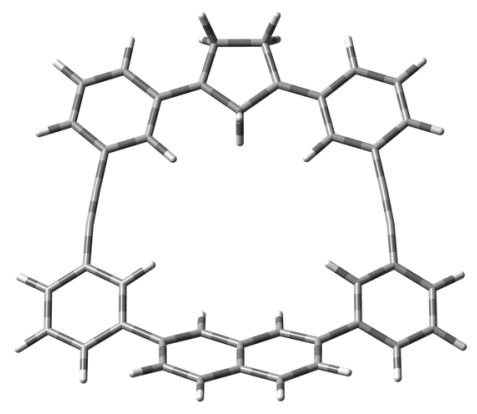

\#p opt freq uwb97xd/6-31g(d)

Charge $=0$, Multiplicity $=3$, Point group $=C_{\mathrm{S}}$

Number of Imaginary Frequencies $=0$

Sum of electronic and zero-point Energies $=-1654.173393$ Hartree

Sum of electronic and thermal Energies $=-1654.140618$ Hartree

Sum of electronic and enthalpy Energies = -1654.139673 Hartree

Sum of electronic and thermal Free Energies $=-1654.239915$ Hartree

\begin{tabular}{|c|c|c|c|c|c|c|c|}
\hline \multirow[b]{2}{*}{ Atom } & \multicolumn{3}{|c|}{ Cartesian Coordinates } & \multicolumn{4}{|c|}{ Cartesian Coordinates } \\
\hline & $X$ & $Y$ & $Z$ & Atom & $X$ & $Y$ & $Z$ \\
\hline $\mathrm{C}$ & 0.156280 & 4.939654 & 1.192510 & $\mathrm{H}$ & 0.248244 & -1.554894 & -2.770423 \\
\hline $\mathrm{C}$ & 0.103270 & 4.028453 & 0.000000 & $\mathrm{H}$ & -1.028663 & -3.831151 & -6.962040 \\
\hline $\mathrm{C}$ & 0.156280 & 4.939654 & -1.192510 & $\mathrm{H}$ & -0.630253 & -5.163131 & -4.922938 \\
\hline $\mathrm{C}$ & 0.254376 & 6.380470 & -0.771655 & $\mathrm{C}$ & -0.309934 & -1.297053 & 4.820117 \\
\hline $\mathrm{C}$ & 0.254376 & 6.380470 & 0.771655 & $\mathrm{C}$ & -0.519257 & -4.082866 & 4.891236 \\
\hline $\mathrm{C}$ & 0.114072 & 4.508443 & -2.544697 & $\mathrm{C}$ & -0.058171 & -2.064175 & 3.678010 \\
\hline $\mathrm{C}$ & 0.018638 & 3.661393 & -5.245858 & $\mathrm{C}$ & -0.657629 & -1.943432 & 6.012744 \\
\hline $\mathrm{C}$ & 0.161343 & 5.438480 & -3.615679 & $\mathrm{C}$ & -0.753896 & -3.330152 & 6.038750 \\
\hline $\mathrm{C}$ & 0.020322 & 3.136903 & -2.880743 & $\mathrm{C}$ & -0.172708 & -3.454807 & 3.690359 \\
\hline $\mathrm{C}$ & -0.027695 & 2.713609 & -4.209645 & $\mathrm{H}$ & 0.248244 & -1.554894 & 2.770423 \\
\hline $\mathrm{C}$ & 0.113784 & 5.015424 & -4.933484 & $\mathrm{H}$ & -0.851154 & -1.357931 & 6.905495 \\
\hline $\mathrm{H}$ & 0.235017 & 6.499723 & -3.399748 & $\mathrm{H}$ & -1.028663 & -3.831151 & 6.962040 \\
\hline $\mathrm{H}$ & -0.017506 & 2.388410 & -2.095807 & $\mathrm{H}$ & -0.630253 & -5.163131 & 4.922938 \\
\hline $\mathrm{H}$ & 0.151027 & 5.748459 & -5.733920 & $\mathrm{C}$ & 0.069272 & -4.207168 & 2.433919 \\
\hline $\mathrm{H}$ & -0.018738 & 3.329147 & -6.277724 & $\mathrm{H}$ & -0.875177 & -2.731033 & 1.209634 \\
\hline $\mathrm{C}$ & 0.114072 & 4.508443 & 2.544697 & $\mathrm{C}$ & -0.323554 & -3.667224 & 1.231045 \\
\hline $\mathrm{C}$ & 0.018638 & 3.661393 & 5.245858 & $\mathrm{C}$ & 1.034204 & -6.099678 & 1.250309 \\
\hline $\mathrm{C}$ & 0.020322 & 3.136903 & 2.880743 & $\mathrm{C}$ & -0.008533 & -4.287077 & 0.000000 \\
\hline $\mathrm{C}$ & 0.161343 & 5.438480 & 3.615679 & $\mathrm{C}$ & 0.744787 & -5.458936 & 2.427444 \\
\hline $\mathrm{C}$ & 0.113784 & 5.015424 & 4.933484 & $\mathrm{C}$ & 0.687513 & -5.523416 & 0.000000 \\
\hline $\mathrm{C}$ & -0.027695 & 2.713609 & 4.209645 & $\mathrm{C}$ & -0.323554 & -3.667224 & -1.231045 \\
\hline $\mathrm{H}$ & -0.017506 & 2.388410 & 2.095807 & $\mathrm{H}$ & 1.064714 & -5.892859 & 3.370629 \\
\hline $\mathrm{H}$ & 0.235017 & 6.499723 & 3.399748 & $\mathrm{H}$ & 1.566691 & -7.047237 & -1.263022 \\
\hline $\mathrm{H}$ & 0.151027 & 5.748459 & 5.733920 & $\mathrm{H}$ & 1.566691 & -7.047237 & 1.263022 \\
\hline $\mathrm{H}$ & -0.018738 & 3.329147 & 6.277724 & $\mathrm{C}$ & 0.069272 & -4.207168 & -2.433919 \\
\hline $\mathrm{C}$ & -0.125144 & 1.315640 & 4.501350 & $\mathrm{H}$ & -0.875177 & -2.731033 & -1.209634 \\
\hline $\mathrm{C}$ & -0.211654 & 0.126576 & 4.712456 & $\mathrm{C}$ & 0.744787 & -5.458936 & -2.427444 \\
\hline
\end{tabular}




\begin{tabular}{cccccccc}
\hline & \multicolumn{3}{c}{ Cartesian Coordinates } & \multicolumn{4}{c}{ Cartesian Coordinates } \\
Atom & $\boldsymbol{X}$ & \multicolumn{1}{c}{$\boldsymbol{Y}$} & $\boldsymbol{Z}$ & Atom & $\boldsymbol{X}$ & $\boldsymbol{Y}$ & $\boldsymbol{Z}$ \\
\hline $\mathrm{C}$ & -0.125144 & 1.315640 & -4.501350 & $\mathrm{H}$ & 1.064714 & -5.892859 & -3.370629 \\
$\mathrm{C}$ & -0.211654 & 0.126576 & -4.712456 & $\mathrm{C}$ & 1.034204 & -6.099678 & -1.250309 \\
$\mathrm{C}$ & -0.309934 & -1.297053 & -4.820117 & $\mathrm{H}$ & -0.584433 & 6.967293 & -1.171147 \\
$\mathrm{C}$ & -0.519257 & -4.082866 & -4.891236 & $\mathrm{H}$ & -0.584433 & 6.967293 & 1.171147 \\
$\mathrm{C}$ & -0.657629 & -1.943432 & -6.012744 & $\mathrm{H}$ & 1.164608 & 6.848513 & -1.171825 \\
$\mathrm{C}$ & -0.058171 & -2.064175 & -3.678010 & $\mathrm{H}$ & 1.164608 & 6.848513 & 1.171825 \\
$\mathrm{C}$ & -0.172708 & -3.454807 & -3.690359 & $\mathrm{H}$ & -0.810312 & 3.407221 & 0.000000 \\
$\mathrm{C}$ & -0.753896 & -3.330152 & -6.038750 & $\mathrm{H}$ & 0.938819 & 3.305188 & 0.000000 \\
$\mathrm{H}$ & -0.851154 & -1.357931 & -6.905495 & & & & \\
\hline
\end{tabular}




\section{DR3H0}

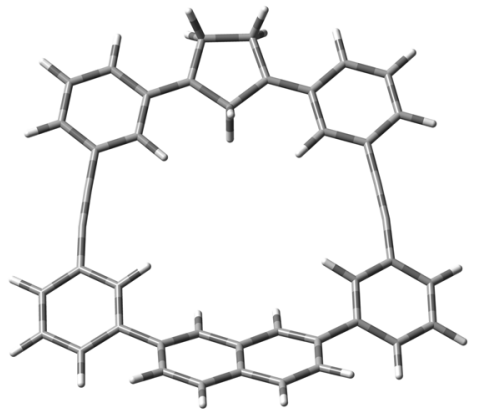

\#p opt freq um062x/6-31g(d)

Charge $=0$, Multiplicity $=3$, Point group $=C_{\mathrm{S}}$

Number of Imaginary Frequencies $=0$

Sum of electronic and zero-point Energies $=-1654.044096$ Hartree

Sum of electronic and thermal Energies $=-1654.011226$ Hartree

Sum of electronic and enthalpy Energies = -1654.010282 Hartree

Sum of electronic and thermal Free Energies $=-1654.110199$ Hartree

\begin{tabular}{|c|c|c|c|c|c|c|c|}
\hline \multirow[b]{2}{*}{ Atom } & \multicolumn{3}{|c|}{ Cartesian Coordinates } & \multicolumn{4}{|c|}{ Cartesian Coordinates } \\
\hline & $X$ & $Y$ & $Z$ & Atom & $X$ & $Y$ & $Z$ \\
\hline $\mathrm{C}$ & 0.147360 & 4.943423 & 1.189862 & $\mathrm{H}$ & 0.235568 & -1.552299 & -2.764235 \\
\hline $\mathrm{C}$ & 0.098036 & 4.030048 & 0.000000 & $\mathrm{H}$ & -0.963628 & -3.831483 & -6.978769 \\
\hline $\mathrm{C}$ & 0.147360 & 4.943423 & -1.189862 & $\mathrm{H}$ & -0.594571 & -5.163670 & -4.931921 \\
\hline $\mathrm{C}$ & 0.238107 & 6.384770 & -0.772093 & $\mathrm{C}$ & -0.289540 & -1.296178 & 4.823063 \\
\hline $\mathrm{C}$ & 0.238107 & 6.384770 & 0.772093 & $\mathrm{C}$ & -0.487255 & -4.083131 & 4.899765 \\
\hline $\mathrm{C}$ & 0.107726 & 4.511681 & -2.544976 & $\mathrm{C}$ & -0.054789 & -2.062514 & 3.676540 \\
\hline $\mathrm{C}$ & 0.018482 & 3.667636 & -5.245075 & $\mathrm{C}$ & -0.614923 & -1.942831 & 6.022738 \\
\hline $\mathrm{C}$ & 0.151129 & 5.443662 & -3.612421 & $\mathrm{C}$ & -0.705940 & -3.330212 & 6.051056 \\
\hline $\mathrm{C}$ & 0.020943 & 3.141190 & -2.879303 & $\mathrm{C}$ & -0.163425 & -3.454045 & 3.692180 \\
\hline $\mathrm{C}$ & -0.023996 & 2.719474 & -4.209243 & $\mathrm{H}$ & 0.235568 & -1.552299 & 2.764235 \\
\hline $\mathrm{C}$ & 0.106721 & 5.021783 & -4.931544 & $\mathrm{H}$ & -0.794474 & -1.355432 & 6.916848 \\
\hline $\mathrm{H}$ & 0.219479 & 6.504329 & -3.391529 & $\mathrm{H}$ & -0.963628 & -3.831483 & 6.978769 \\
\hline $\mathrm{H}$ & -0.013848 & 2.392240 & -2.094339 & $\mathrm{H}$ & -0.594571 & -5.163670 & 4.931921 \\
\hline $\mathrm{H}$ & 0.140983 & 5.755723 & -5.730831 & $\mathrm{C}$ & 0.061991 & -4.207970 & 2.434615 \\
\hline $\mathrm{H}$ & -0.016700 & 3.333546 & -6.276069 & $\mathrm{H}$ & -0.832637 & -2.700568 & 1.209194 \\
\hline $\mathrm{C}$ & 0.107726 & 4.511681 & 2.544976 & $\mathrm{C}$ & -0.309957 & -3.653216 & 1.231266 \\
\hline $\mathrm{C}$ & 0.018482 & 3.667636 & 5.245075 & $\mathrm{C}$ & 0.969835 & -6.130168 & 1.250669 \\
\hline $\mathrm{C}$ & 0.020943 & 3.141190 & 2.879303 & $\mathrm{C}$ & -0.013206 & -4.282630 & 0.000000 \\
\hline $\mathrm{C}$ & 0.151129 & 5.443662 & 3.612421 & $\mathrm{C}$ & 0.698343 & -5.481880 & 2.428395 \\
\hline $\mathrm{C}$ & 0.106721 & 5.021783 & 4.931544 & $\mathrm{C}$ & 0.642541 & -5.541879 & 0.000000 \\
\hline $\mathrm{C}$ & -0.023996 & 2.719474 & 4.209243 & $\mathrm{C}$ & -0.309957 & -3.653216 & -1.231266 \\
\hline $\mathrm{H}$ & -0.013848 & 2.392240 & 2.094339 & $\mathrm{H}$ & 1.002888 & -5.924543 & 3.372479 \\
\hline $\mathrm{H}$ & 0.219479 & 6.504329 & 3.391529 & $\mathrm{H}$ & 1.472031 & -7.094037 & -1.260790 \\
\hline $\mathrm{H}$ & 0.140983 & 5.755723 & 5.730831 & $\mathrm{H}$ & 1.472031 & -7.094037 & 1.260790 \\
\hline $\mathrm{H}$ & -0.016700 & 3.333546 & 6.276069 & $\mathrm{C}$ & 0.061991 & -4.207970 & -2.434615 \\
\hline $\mathrm{C}$ & -0.114908 & 1.319484 & 4.502093 & $\mathrm{H}$ & -0.832637 & -2.700568 & -1.209194 \\
\hline $\mathrm{C}$ & -0.196051 & 0.129084 & 4.712586 & $\mathrm{C}$ & 0.698343 & -5.481880 & -2.428395 \\
\hline
\end{tabular}




\begin{tabular}{cccccccc}
\hline & \multicolumn{3}{c}{ Cartesian Coordinates } & \multicolumn{4}{c}{ Cartesian Coordinates } \\
Atom & $\boldsymbol{X}$ & \multicolumn{1}{c}{$\boldsymbol{Y}$} & $\boldsymbol{Z}$ & Atom & $\boldsymbol{X}$ & $\boldsymbol{Y}$ & $\boldsymbol{Z}$ \\
\hline $\mathrm{C}$ & -0.114908 & 1.319484 & -4.502093 & $\mathrm{H}$ & 1.002888 & -5.924543 & -3.372479 \\
$\mathrm{C}$ & -0.196051 & 0.129084 & -4.712586 & $\mathrm{C}$ & 0.969835 & -6.130168 & -1.250669 \\
$\mathrm{C}$ & -0.289540 & -1.296178 & -4.823063 & $\mathrm{H}$ & -0.603473 & 6.966191 & -1.172928 \\
$\mathrm{C}$ & -0.487255 & -4.083131 & -4.899765 & $\mathrm{H}$ & -0.603473 & 6.966191 & 1.172928 \\
$\mathrm{C}$ & -0.614923 & -1.942831 & -6.022738 & $\mathrm{H}$ & 1.145488 & 6.856532 & -1.173633 \\
$\mathrm{C}$ & -0.054789 & -2.062514 & -3.676540 & $\mathrm{H}$ & 1.145488 & 6.856532 & 1.173633 \\
$\mathrm{C}$ & -0.163425 & -3.454045 & -3.692180 & $\mathrm{H}$ & -0.812809 & 3.404071 & 0.000000 \\
$\mathrm{C}$ & -0.705940 & -3.330212 & -6.051056 & $\mathrm{H}$ & 0.936108 & 3.309120 & 0.000000 \\
$\mathrm{H}$ & -0.794474 & -1.355432 & -6.916848 & & & & \\
\hline
\end{tabular}




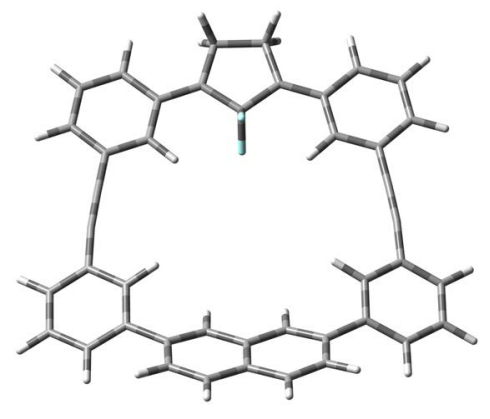

\#p opt freq ub3lyp/6-31g(d)

Charge $=0$, Multiplicity $=3$, Point group $=C_{\mathrm{S}}$

Number of Imaginary Frequencies $=1, v_{i}=-25.89$

Sum of electronic and zero-point Energies $=-1853.235354$ Hartree

Sum of electronic and thermal Energies $=-1853.201426$ Hartree

Sum of electronic and enthalpy Energies = -1853.200482 Hartree

Sum of electronic and thermal Free Energies $=-1853.301606$ Hartree

\begin{tabular}{|c|c|c|c|c|c|c|c|}
\hline \multirow[b]{2}{*}{ Atom } & \multicolumn{3}{|c|}{ Cartesian Coordinates } & \multicolumn{4}{|c|}{ Cartesian Coordinates } \\
\hline & $X$ & $Y$ & $Z$ & Atom & $X$ & $Y$ & $Z$ \\
\hline $\mathrm{C}$ & -0.141939 & -4.810087 & 1.206156 & $\mathrm{H}$ & -0.223240 & 1.729319 & -2.772732 \\
\hline $\mathrm{C}$ & -0.090615 & -3.916575 & 0.000000 & $\mathrm{H}$ & 0.930260 & 4.003040 & -7.009433 \\
\hline $\mathrm{C}$ & -0.141939 & -4.810087 & -1.206156 & $\mathrm{H}$ & 0.581411 & 5.340905 & -4.964353 \\
\hline $\mathrm{C}$ & -0.230834 & -6.249708 & -0.776947 & $\mathrm{C}$ & 0.281651 & 1.462459 & 4.837558 \\
\hline $\mathrm{C}$ & -0.230834 & -6.249708 & 0.776947 & $\mathrm{C}$ & 0.474710 & 4.260561 & 4.923334 \\
\hline $\mathrm{C}$ & -0.102871 & -4.355900 & -2.553123 & $\mathrm{C}$ & 0.057010 & 2.238735 & 3.687869 \\
\hline $\mathrm{C}$ & -0.012546 & -3.497561 & -5.255430 & $\mathrm{C}$ & 0.594051 & 2.112413 & 6.046341 \\
\hline $\mathrm{C}$ & -0.142580 & -5.286604 & -3.630722 & $\mathrm{C}$ & 0.682909 & 3.502480 & 6.077159 \\
\hline $\mathrm{C}$ & -0.020215 & -2.975440 & -2.881349 & $\mathrm{C}$ & 0.162340 & 3.635344 & 3.704138 \\
\hline $\mathrm{C}$ & 0.025645 & -2.544125 & -4.213352 & $\mathrm{H}$ & -0.223240 & 1.729319 & 2.772732 \\
\hline $\mathrm{C}$ & -0.097429 & -4.856738 & -4.949666 & $\mathrm{H}$ & 0.765184 & 1.525880 & 6.943510 \\
\hline $\mathrm{H}$ & -0.207500 & -6.348797 & -3.417719 & $\mathrm{H}$ & 0.930260 & 4.003040 & 7.009433 \\
\hline $\mathrm{H}$ & 0.010352 & -2.237195 & -2.090796 & $\mathrm{H}$ & 0.581411 & 5.340905 & 4.964353 \\
\hline $\mathrm{H}$ & -0.128143 & -5.586733 & -5.754051 & $\mathrm{C}$ & -0.055287 & 4.393745 & 2.445782 \\
\hline $\mathrm{H}$ & 0.023131 & -3.161792 & -6.286673 & $\mathrm{H}$ & 0.817960 & 2.876473 & 1.207876 \\
\hline $\mathrm{C}$ & -0.102871 & -4.355900 & 2.553123 & $\mathrm{C}$ & 0.307600 & 3.835109 & 1.232824 \\
\hline $\mathrm{C}$ & -0.012546 & -3.497561 & 5.255430 & $\mathrm{C}$ & -0.940746 & 6.333043 & 1.253485 \\
\hline $\mathrm{C}$ & -0.020215 & -2.975440 & 2.881349 & $\mathrm{C}$ & 0.017947 & 4.466607 & 0.000000 \\
\hline $\mathrm{C}$ & -0.142580 & -5.286604 & 3.630722 & $\mathrm{C}$ & -0.674740 & 5.678540 & 2.435511 \\
\hline $\mathrm{C}$ & -0.097429 & -4.856738 & 4.949666 & $\mathrm{C}$ & -0.624760 & 5.743250 & 0.000000 \\
\hline $\mathrm{C}$ & 0.025645 & -2.544125 & 4.213352 & $\mathrm{C}$ & 0.307600 & 3.835109 & -1.232824 \\
\hline $\mathrm{H}$ & 0.010352 & -2.237195 & 2.090796 & $\mathrm{H}$ & -0.973696 & 6.130062 & 3.377310 \\
\hline $\mathrm{H}$ & -0.207500 & -6.348797 & 3.417719 & $\mathrm{H}$ & -1.429905 & 7.304312 & -1.268948 \\
\hline $\mathrm{H}$ & -0.128143 & -5.586733 & 5.754051 & $\mathrm{H}$ & -1.429905 & 7.304312 & 1.268948 \\
\hline $\mathrm{H}$ & 0.023131 & -3.161792 & 6.286673 & $\mathrm{C}$ & -0.055287 & 4.393745 & -2.445782 \\
\hline $\mathrm{C}$ & 0.112131 & -1.150250 & 4.504542 & $\mathrm{H}$ & 0.817960 & 2.876473 & -1.207876 \\
\hline $\mathrm{C}$ & 0.191435 & 0.043408 & 4.724204 & $\mathrm{C}$ & -0.674740 & 5.678540 & -2.435511 \\
\hline
\end{tabular}




\begin{tabular}{cccc|cccc}
\hline & \multicolumn{3}{c}{ Cartesian Coordinates } & \multicolumn{4}{c}{ Cartesian Coordinates } \\
Atom & $\boldsymbol{X}$ & $\boldsymbol{Y}$ & $\boldsymbol{Z}$ & Atom & $\boldsymbol{X}$ & $\boldsymbol{Y}$ & $\boldsymbol{Z}$ \\
\hline $\mathrm{C}$ & 0.112131 & -1.150250 & -4.504542 & $\mathrm{H}$ & -0.973696 & 6.130062 & -3.377310 \\
$\mathrm{C}$ & 0.191435 & 0.043408 & -4.724204 & $\mathrm{C}$ & -0.940746 & 6.333043 & -1.253485 \\
$\mathrm{C}$ & 0.281651 & 1.462459 & -4.837558 & $\mathrm{H}$ & 0.610686 & -6.836266 & -1.172398 \\
$\mathrm{C}$ & 0.474710 & 4.260561 & -4.923334 & $\mathrm{H}$ & 0.610686 & -6.836266 & 1.172398 \\
$\mathrm{C}$ & 0.594051 & 2.112413 & -6.046341 & $\mathrm{H}$ & -1.137788 & -6.728627 & -1.172852 \\
$\mathrm{C}$ & 0.057010 & 2.238735 & -3.687869 & $\mathrm{H}$ & -1.137788 & -6.728627 & 1.172852 \\
$\mathrm{C}$ & 0.162340 & 3.635344 & -3.704138 & $\mathrm{~F}$ & -1.132309 & -2.992808 & 0.000000 \\
$\mathrm{C}$ & 0.682909 & 3.502480 & -6.077159 & $\mathrm{~F}$ & 1.050487 & -3.119853 & 0.000000 \\
$\mathrm{H}$ & 0.765184 & 1.525880 & -6.943510 & & & & \\
\hline
\end{tabular}




\section{DR3F0}

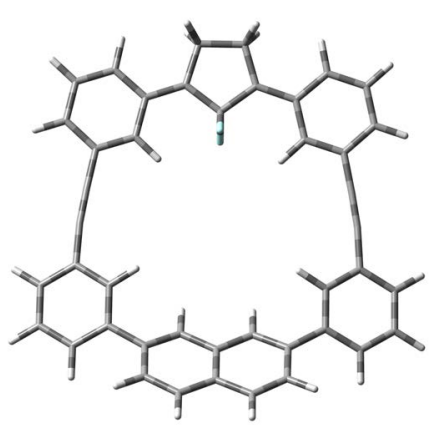

\#p opt freq uwb97xd/6-31g(d)

Charge $=0$, Multiplicity $=3$, Point group $=C_{\mathrm{S}}$

Number of Imaginary Frequencies $=1, v_{i}=-15.94$

Sum of electronic and zero-point Energies $=-1852.612332$ Hartree

Sum of electronic and thermal Energies $=-1852.578750$ Hartree

Sum of electronic and enthalpy Energies = -1852.577806 Hartree

Sum of electronic and thermal Free Energies $=-1852.678260$ Hartree

\begin{tabular}{|c|c|c|c|c|c|c|c|}
\hline \multirow[b]{2}{*}{ Atom } & \multicolumn{3}{|c|}{ Cartesian Coordinates } & \multicolumn{4}{|c|}{ Cartesian Coordinates } \\
\hline & $X$ & $Y$ & $Z$ & Atom & $X$ & $Y$ & $Z$ \\
\hline $\mathrm{C}$ & -0.149010 & -4.778563 & 1.201464 & $\mathrm{H}$ & -0.248387 & 1.718752 & -2.776315 \\
\hline $\mathrm{C}$ & -0.090580 & -3.881834 & 0.000000 & $\mathrm{H}$ & 1.031350 & 4.005922 & -6.961995 \\
\hline $\mathrm{C}$ & -0.149010 & -4.778563 & -1.201464 & $\mathrm{H}$ & 0.632675 & 5.332317 & -4.919671 \\
\hline $\mathrm{C}$ & -0.248030 & -6.213399 & -0.773780 & $\mathrm{C}$ & 0.310216 & 1.467353 & 4.826703 \\
\hline $\mathrm{C}$ & -0.248030 & -6.213399 & 0.773780 & $\mathrm{C}$ & 0.520862 & 4.252056 & 4.890743 \\
\hline $\mathrm{C}$ & -0.109313 & -4.331388 & -2.548826 & $\mathrm{C}$ & 0.057998 & 2.230889 & 3.682298 \\
\hline $\mathrm{C}$ & -0.022105 & -3.492396 & -5.245365 & $\mathrm{C}$ & 0.658655 & 2.115862 & 6.017798 \\
\hline $\mathrm{C}$ & -0.160554 & -5.265317 & -3.614774 & $\mathrm{C}$ & 0.755727 & 3.502537 & 6.040265 \\
\hline $\mathrm{C}$ & -0.015735 & -2.958557 & -2.881653 & $\mathrm{C}$ & 0.173115 & 3.621505 & 3.691423 \\
\hline $\mathrm{C}$ & 0.028115 & -2.541426 & -4.211646 & $\mathrm{H}$ & -0.248387 & 1.718752 & 2.776315 \\
\hline $\mathrm{C}$ & -0.116938 & -4.845906 & -4.933868 & $\mathrm{H}$ & 0.852501 & 1.532377 & 6.911814 \\
\hline $\mathrm{H}$ & -0.233996 & -6.325777 & -3.395552 & $\mathrm{H}$ & 1.031350 & 4.005922 & 6.961995 \\
\hline $\mathrm{H}$ & 0.024808 & -2.213351 & -2.097424 & $\mathrm{H}$ & 0.632675 & 5.332317 & 4.919671 \\
\hline $\mathrm{H}$ & -0.157001 & -5.579759 & -5.733084 & $\mathrm{C}$ & -0.069047 & 4.372041 & 2.433911 \\
\hline $\mathrm{H}$ & 0.012285 & -3.161453 & -6.277854 & $\mathrm{H}$ & 0.867088 & 2.890407 & 1.209271 \\
\hline $\mathrm{C}$ & -0.109313 & -4.331388 & 2.548826 & $\mathrm{C}$ & 0.319926 & 3.829166 & 1.231070 \\
\hline $\mathrm{C}$ & -0.022105 & -3.492396 & 5.245365 & $\mathrm{C}$ & -1.027995 & 6.267480 & 1.250158 \\
\hline $\mathrm{C}$ & -0.015735 & -2.958557 & 2.881653 & $\mathrm{C}$ & 0.006754 & 4.450094 & 0.000000 \\
\hline $\mathrm{C}$ & -0.160554 & -5.265317 & 3.614774 & $\mathrm{C}$ & -0.739987 & 5.626267 & 2.427303 \\
\hline $\mathrm{C}$ & -0.116938 & -4.845906 & 4.933868 & $\mathrm{C}$ & -0.683949 & 5.689454 & 0.000000 \\
\hline $\mathrm{C}$ & 0.028115 & -2.541426 & 4.211646 & $\mathrm{C}$ & 0.319926 & 3.829166 & -1.231070 \\
\hline $\mathrm{H}$ & 0.024808 & -2.213351 & 2.097424 & $\mathrm{H}$ & -1.057625 & 6.061999 & 3.370439 \\
\hline $\mathrm{H}$ & -0.233996 & -6.325777 & 3.395552 & $\mathrm{H}$ & -1.556947 & 7.217030 & -1.262755 \\
\hline $\mathrm{H}$ & -0.157001 & -5.579759 & 5.733084 & $\mathrm{H}$ & -1.556947 & 7.217030 & 1.262755 \\
\hline $\mathrm{H}$ & 0.012285 & -3.161453 & 6.277854 & $\mathrm{C}$ & -0.069047 & 4.372041 & -2.433911 \\
\hline $\mathrm{C}$ & 0.125344 & -1.144922 & 4.508564 & $\mathrm{H}$ & 0.867088 & 2.890407 & -1.209271 \\
\hline $\mathrm{C}$ & 0.211931 & 0.043552 & 4.721537 & $\mathrm{C}$ & -0.739987 & 5.626267 & -2.427303 \\
\hline
\end{tabular}




\begin{tabular}{cccccccc}
\hline & \multicolumn{3}{c}{ Cartesian Coordinates } & \multicolumn{4}{c}{ Cartesian Coordinates } \\
Atom & $\boldsymbol{X}$ & $\boldsymbol{Y}$ & $\boldsymbol{Z}$ & Atom & $\boldsymbol{X}$ & $\boldsymbol{Y}$ & $\boldsymbol{Z}$ \\
\hline $\mathrm{C}$ & 0.125344 & -1.144922 & -4.508564 & $\mathrm{H}$ & -1.057625 & 6.061999 & -3.370439 \\
$\mathrm{C}$ & 0.211931 & 0.043552 & -4.721537 & $\mathrm{C}$ & -1.027995 & 6.267480 & -1.250158 \\
$\mathrm{C}$ & 0.310216 & 1.467353 & -4.826703 & $\mathrm{H}$ & 0.590736 & -6.800856 & -1.170374 \\
$\mathrm{C}$ & 0.520862 & 4.252056 & -4.890743 & $\mathrm{H}$ & 0.590736 & -6.800856 & 1.170374 \\
$\mathrm{C}$ & 0.658655 & 2.115862 & -6.017798 & $\mathrm{H}$ & -1.159123 & -6.680369 & -1.171041 \\
$\mathrm{C}$ & 0.057998 & 2.230889 & -3.682298 & $\mathrm{H}$ & -1.159123 & -6.680369 & 1.171041 \\
$\mathrm{C}$ & 0.173115 & 3.621505 & -3.691423 & $\mathrm{~F}$ & -1.120262 & -2.963136 & 0.000000 \\
$\mathrm{C}$ & 0.755727 & 3.502537 & -6.040265 & $\mathrm{~F}$ & 1.050465 & -3.106060 & 0.000000 \\
$\mathrm{H}$ & 0.852501 & 1.532377 & -6.911814 & & & & \\
\hline
\end{tabular}




\section{DR3F0}

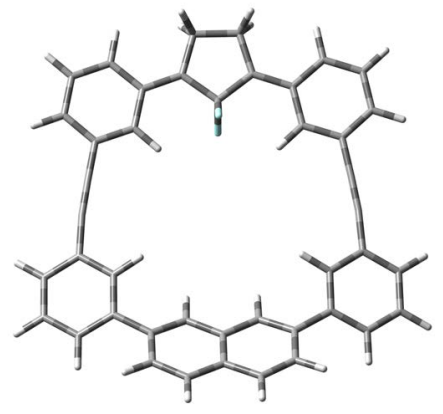

\#p opt freq um062x/6-31g(d)

Charge $=0$, Multiplicity $=3$, Point group $=C_{\mathrm{S}}$

Number of Imaginary Frequencies $=1, v_{i}=-4.43$

Sum of electronic and zero-point Energies $=-1852.478998$ Hartree

Sum of electronic and thermal Energies $=-1852.445430$ Hartree

Sum of electronic and enthalpy Energies $=-1852.444486$ Hartree

Sum of electronic and thermal Free Energies $=-1852.544492$ Hartree

\begin{tabular}{|c|c|c|c|c|c|c|c|}
\hline \multicolumn{5}{|c|}{ Cartesian Coordinates } & \multicolumn{3}{|c|}{ Cartesian Coordinates } \\
\hline Atom & $X$ & $Y$ & $Z$ & Atom & $X$ & $Y$ & $Z$ \\
\hline $\mathrm{C}$ & -0.140711 & -4.809055 & 1.198421 & $\mathrm{H}$ & -0.234092 & 1.732896 & -2.752786 \\
\hline $\mathrm{C}$ & -0.088607 & -3.909727 & 0.000000 & $\mathrm{H}$ & 0.954572 & 3.994583 & -6.980163 \\
\hline $\mathrm{C}$ & -0.140711 & -4.809055 & -1.198421 & $\mathrm{H}$ & 0.591629 & 5.335274 & -4.937924 \\
\hline $\mathrm{C}$ & -0.230140 & -6.244765 & -0.774752 & $\mathrm{C}$ & 0.285822 & 1.469215 & 4.811619 \\
\hline $\mathrm{C}$ & -0.230140 & -6.244765 & 0.774752 & $\mathrm{C}$ & 0.483944 & 4.254918 & 4.900846 \\
\hline $\mathrm{C}$ & -0.102715 & -4.350311 & -2.544331 & $\mathrm{C}$ & 0.053824 & 2.239815 & 3.667641 \\
\hline $\mathrm{C}$ & -0.018747 & -3.485619 & -5.227670 & $\mathrm{C}$ & 0.608164 & 2.110019 & 6.015160 \\
\hline $\mathrm{C}$ & -0.146795 & -5.275404 & -3.615544 & $\mathrm{C}$ & 0.699247 & 3.497232 & 6.049699 \\
\hline $\mathrm{C}$ & -0.017941 & -2.974096 & -2.857544 & $\mathrm{C}$ & 0.163301 & 3.631271 & 3.689628 \\
\hline $\mathrm{C}$ & 0.024299 & -2.544468 & -4.184314 & $\mathrm{H}$ & -0.234092 & 1.732896 & 2.752786 \\
\hline $\mathrm{C}$ & -0.104885 & -4.842858 & -4.931313 & $\mathrm{H}$ & 0.785550 & 1.518714 & 6.907137 \\
\hline $\mathrm{H}$ & -0.213422 & -6.337574 & -3.401344 & $\mathrm{H}$ & 0.954572 & 3.994583 & 6.980163 \\
\hline $\mathrm{H}$ & 0.016852 & -2.239516 & -2.061688 & $\mathrm{H}$ & 0.591629 & 5.335274 & 4.937924 \\
\hline $\mathrm{H}$ & -0.139303 & -5.568331 & -5.737960 & $\mathrm{C}$ & -0.057443 & 4.389522 & 2.433931 \\
\hline $\mathrm{H}$ & 0.014632 & -3.141025 & -6.255432 & $\mathrm{H}$ & 0.835665 & 2.881679 & 1.207435 \\
\hline $\mathrm{C}$ & -0.102715 & -4.350311 & 2.544331 & $\mathrm{C}$ & 0.315576 & 3.835640 & 1.230617 \\
\hline $\mathrm{C}$ & -0.018747 & -3.485619 & 5.227670 & $\mathrm{C}$ & -0.959455 & 6.315144 & 1.250942 \\
\hline $\mathrm{C}$ & -0.017941 & -2.974096 & 2.857544 & $\mathrm{C}$ & 0.020787 & 4.466721 & 0.000000 \\
\hline $\mathrm{C}$ & -0.146795 & -5.275404 & 3.615544 & $\mathrm{C}$ & -0.690712 & 5.664956 & 2.428364 \\
\hline $\mathrm{C}$ & -0.104885 & -4.842858 & 4.931313 & $\mathrm{C}$ & -0.632763 & 5.727007 & 0.000000 \\
\hline $\mathrm{C}$ & 0.024299 & -2.544468 & 4.184314 & $\mathrm{C}$ & 0.315576 & 3.835640 & -1.230617 \\
\hline $\mathrm{H}$ & 0.016852 & -2.239516 & 2.061688 & $\mathrm{H}$ & -0.995738 & 6.107341 & 3.372450 \\
\hline $\mathrm{H}$ & -0.213422 & -6.337574 & 3.401344 & $\mathrm{H}$ & -1.459780 & 7.279987 & -1.261601 \\
\hline $\mathrm{H}$ & -0.139303 & -5.568331 & 5.737960 & $\mathrm{H}$ & -1.459780 & 7.279987 & 1.261601 \\
\hline $\mathrm{H}$ & 0.014632 & -3.141025 & 6.255432 & $\mathrm{C}$ & -0.057443 & 4.389522 & -2.433931 \\
\hline $\mathrm{C}$ & 0.113198 & -1.144222 & 4.474696 & $\mathrm{H}$ & 0.835665 & 2.881679 & -1.207435 \\
\hline $\mathrm{C}$ & 0.192908 & 0.044521 & 4.693673 & $\mathrm{C}$ & -0.690712 & 5.664956 & -2.428364 \\
\hline
\end{tabular}




\begin{tabular}{cccccccc}
\hline & \multicolumn{3}{c}{ Cartesian Coordinates } & \multicolumn{4}{c}{ Cartesian Coordinates } \\
Atom & $\boldsymbol{X}$ & \multicolumn{1}{c}{$\boldsymbol{Y}$} & $\boldsymbol{Z}$ & Atom & $\boldsymbol{X}$ & $\boldsymbol{Y}$ & $\boldsymbol{Z}$ \\
\hline $\mathrm{C}$ & 0.113198 & -1.144222 & -4.474696 & $\mathrm{H}$ & -0.995738 & 6.107341 & -3.372450 \\
$\mathrm{C}$ & 0.192908 & 0.044521 & -4.693673 & $\mathrm{C}$ & -0.959455 & 6.315144 & -1.250942 \\
$\mathrm{C}$ & 0.285822 & 1.469215 & -4.811619 & $\mathrm{H}$ & 0.612283 & -6.825523 & -1.172769 \\
$\mathrm{C}$ & 0.483944 & 4.254918 & -4.900846 & $\mathrm{H}$ & 0.612283 & -6.825523 & 1.172769 \\
$\mathrm{C}$ & 0.608164 & 2.110019 & -6.015160 & $\mathrm{H}$ & -1.137675 & -6.716862 & -1.173421 \\
$\mathrm{C}$ & 0.053824 & 2.239815 & -3.667641 & $\mathrm{H}$ & -1.137675 & -6.716862 & 1.173421 \\
$\mathrm{C}$ & 0.163301 & 3.631271 & -3.689628 & $\mathrm{~F}$ & -1.120316 & -2.999438 & 0.000000 \\
$\mathrm{C}$ & 0.699247 & 3.497232 & -6.049699 & $\mathrm{~F}$ & 1.041679 & -3.125534 & 0.000000 \\
$\mathrm{H}$ & 0.785550 & 1.518714 & -6.907137 & & & & \\
\hline
\end{tabular}




\section{DR3H1}

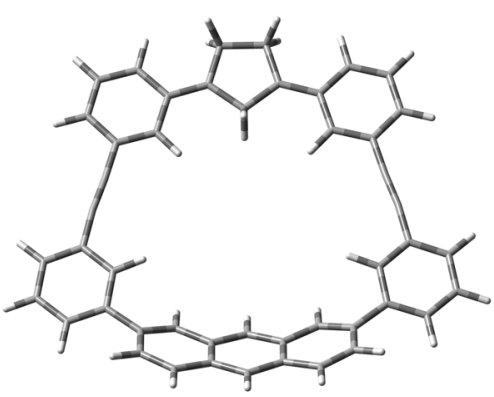

\#p opt freq ub3lyp/6-31g(d)

Charge $=0$, Multiplicity $=3$, Point group $=C_{\mathrm{S}}$

Number of Imaginary Frequencies $=1, v_{i}=-15.73$

Sum of electronic and zero-point Energies $=-1808.329455$ Hartree

Sum of electronic and thermal Energies $=-1808.294527$ Hartree

Sum of electronic and enthalpy Energies = -1808.293583 Hartree

Sum of electronic and thermal Free Energies $=-1808.396372$ Hartree

\begin{tabular}{|c|c|c|c|c|c|c|c|}
\hline \multirow[b]{2}{*}{ Atom } & \multicolumn{3}{|c|}{ Cartesian Coordinates } & \multicolumn{4}{|c|}{ Cartesian Coordinates } \\
\hline & $X$ & $Y$ & $Z$ & Atom & $X$ & $Y$ & $Z$ \\
\hline $\mathrm{C}$ & 0.121216 & 5.291491 & 1.201513 & $\mathrm{H}$ & -0.346839 & -4.501922 & 6.341653 \\
\hline $\mathrm{C}$ & 0.184412 & 6.736746 & 0.771862 & $\mathrm{C}$ & -0.161001 & -0.688621 & -5.549288 \\
\hline $\mathrm{C}$ & 0.184412 & 6.736746 & -0.771862 & $\mathrm{C}$ & -0.279154 & -3.441878 & -6.112190 \\
\hline $\mathrm{C}$ & 0.121216 & 5.291491 & -1.201513 & $\mathrm{C}$ & -0.093254 & -1.643912 & -4.522958 \\
\hline $\mathrm{H}$ & -0.666772 & 7.308942 & 1.169614 & $\mathrm{C}$ & -0.283579 & -1.135580 & -6.879040 \\
\hline $\mathrm{H}$ & -0.666772 & 7.308942 & -1.169614 & $\mathrm{C}$ & -0.335827 & -2.502978 & -7.145292 \\
\hline $\mathrm{C}$ & 0.098230 & 4.900410 & 2.571540 & $\mathrm{C}$ & -0.167458 & -3.020064 & -4.777514 \\
\hline $\mathrm{C}$ & 0.050605 & 4.211228 & 5.331205 & $\mathrm{H}$ & 0.046037 & -1.294075 & -3.506564 \\
\hline $\mathrm{C}$ & 0.039326 & 3.542543 & 2.990472 & $\mathrm{H}$ & -0.331797 & -0.412767 & -7.687444 \\
\hline $\mathrm{C}$ & 0.133532 & 5.892579 & 3.596206 & $\mathrm{H}$ & -0.431734 & -2.843493 & -8.172815 \\
\hline $\mathrm{C}$ & 0.109757 & 5.547950 & 4.939552 & $\mathrm{H}$ & -0.346839 & -4.501922 & -6.341653 \\
\hline $\mathrm{C}$ & 0.015355 & 3.194639 & 4.350195 & $\mathrm{C}$ & -0.105224 & -3.943142 & 3.616960 \\
\hline $\mathrm{H}$ & 0.011744 & 2.750077 & 2.249213 & $\mathrm{H}$ & -1.309600 & -2.668971 & 2.395297 \\
\hline $\mathrm{H}$ & 0.179570 & 6.940837 & 3.321943 & $\mathrm{C}$ & -0.695925 & -3.564483 & 2.433177 \\
\hline $\mathrm{H}$ & 0.137546 & 6.328768 & 5.695130 & $\mathrm{C}$ & 0.852927 & -5.893264 & 2.492600 \\
\hline $\mathrm{H}$ & 0.031904 & 3.937174 & 6.380785 & $\mathrm{C}$ & -0.459391 & -4.257521 & 1.216042 \\
\hline $\mathrm{C}$ & 0.098230 & 4.900410 & -2.571540 & $\mathrm{C}$ & 0.642542 & -5.168309 & 3.638076 \\
\hline $\mathrm{C}$ & 0.050605 & 4.211228 & -5.331205 & $\mathrm{C}$ & 0.366768 & -5.439813 & 1.224157 \\
\hline $\mathrm{C}$ & 0.133532 & 5.892579 & -3.596206 & $\mathrm{C}$ & -0.900197 & -3.731811 & 0.000000 \\
\hline $\mathrm{C}$ & 0.039326 & 3.542543 & -2.990472 & $\mathrm{H}$ & 1.090696 & -5.495838 & 4.572323 \\
\hline $\mathrm{C}$ & 0.015355 & 3.194639 & -4.350195 & $\mathrm{H}$ & 1.352924 & -6.914900 & 0.000000 \\
\hline $\mathrm{C}$ & 0.109757 & 5.547950 & -4.939552 & $\mathrm{H}$ & 1.452805 & -6.799756 & 2.526390 \\
\hline $\mathrm{H}$ & 0.179570 & 6.940837 & -3.321943 & $\mathrm{C}$ & -0.459391 & -4.257521 & -1.216042 \\
\hline $\mathrm{H}$ & 0.011744 & 2.750077 & -2.249213 & $\mathrm{H}$ & -1.508899 & -2.830117 & 0.000000 \\
\hline $\mathrm{H}$ & 0.137546 & 6.328768 & -5.695130 & $\mathrm{C}$ & -0.695925 & -3.564483 & -2.433177 \\
\hline $\mathrm{H}$ & 0.031904 & 3.937174 & -6.380785 & $\mathrm{C}$ & 0.366768 & -5.439813 & -1.224157 \\
\hline $\mathrm{C}$ & -0.044857 & 1.831673 & 4.770244 & $\mathrm{H}$ & 1.452805 & -6.799756 & -2.526390 \\
\hline
\end{tabular}




\begin{tabular}{cccccccc}
\hline & \multicolumn{3}{c}{ Cartesian Coordinates } & \multicolumn{4}{c}{ Cartesian Coordinates } \\
Atom & $\boldsymbol{X}$ & $\boldsymbol{Y}$ & $\boldsymbol{Z}$ & Atom & $\boldsymbol{X}$ & $\boldsymbol{Y}$ & $\boldsymbol{Z}$ \\
\hline $\mathrm{C}$ & -0.097117 & 0.691496 & 5.192716 & $\mathrm{C}$ & 0.721922 & -6.028173 & 0.000000 \\
$\mathrm{C}$ & -0.044857 & 1.831673 & -4.770244 & $\mathrm{C}$ & -0.105224 & -3.943142 & -3.616960 \\
$\mathrm{C}$ & -0.097117 & 0.691496 & -5.192716 & $\mathrm{H}$ & -1.309600 & -2.668971 & -2.395297 \\
$\mathrm{C}$ & -0.161001 & -0.688621 & 5.549288 & $\mathrm{C}$ & 0.642542 & -5.168309 & -3.638076 \\
$\mathrm{C}$ & -0.279154 & -3.441878 & 6.112190 & $\mathrm{H}$ & 1.090696 & -5.495838 & -4.572323 \\
$\mathrm{C}$ & -0.093254 & -1.643912 & 4.522958 & $\mathrm{C}$ & 0.852927 & -5.893264 & -2.492600 \\
$\mathrm{C}$ & -0.283579 & -1.135580 & 6.879040 & $\mathrm{H}$ & 1.082090 & 7.232628 & 1.170003 \\
$\mathrm{C}$ & -0.335827 & -2.502978 & 7.145292 & $\mathrm{H}$ & 1.082090 & 7.232628 & -1.170003 \\
$\mathrm{C}$ & -0.167458 & -3.020064 & 4.777514 & $\mathrm{C}$ & 0.085564 & 4.375434 & 0.000000 \\
$\mathrm{H}$ & 0.046037 & -1.294075 & 3.506564 & $\mathrm{H}$ & 0.932428 & 3.663353 & 0.000000 \\
$\mathrm{H}$ & -0.331797 & -0.412767 & 7.687444 & $\mathrm{H}$ & -0.814053 & 3.731474 & 0.000000 \\
$\mathrm{H}$ & -0.431734 & -2.843493 & 8.172815 & & & & \\
\hline
\end{tabular}




\section{DR3H1}

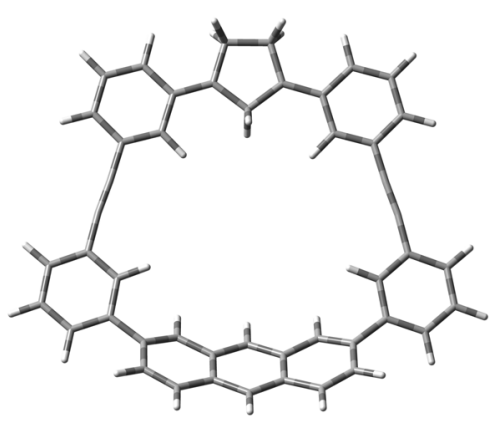

\#p opt freq uwb97xd/6-31g(d)

Charge $=0$, Multiplicity $=3$, Point group $=C_{\mathrm{S}}$

Number of Imaginary Frequencies $=1, v_{i}=-7.74$

Sum of electronic and zero-point Energies $=-1807.701838$ Hartree

Sum of electronic and thermal Energies $=-1807.667356$ Hartree

Sum of electronic and enthalpy Energies = -1807.666411 Hartree

Sum of electronic and thermal Free Energies $=-1807.768189$ Hartree

\begin{tabular}{|c|c|c|c|c|c|c|c|}
\hline \multirow[b]{2}{*}{ Atom } & \multicolumn{3}{|c|}{ Cartesian Coordinates } & \multicolumn{4}{|c|}{ Cartesian Coordinates } \\
\hline & $X$ & $Y$ & $Z$ & Atom & $X$ & $Y$ & $Z$ \\
\hline $\mathrm{C}$ & 0.131336 & 5.288103 & 1.194845 & $\mathrm{H}$ & -0.397913 & -4.493649 & 6.309184 \\
\hline $\mathrm{C}$ & 0.202657 & 6.729633 & 0.769116 & $\mathrm{C}$ & -0.184603 & -0.695705 & -5.514119 \\
\hline $\mathrm{C}$ & 0.202657 & 6.729633 & -0.769116 & $\mathrm{C}$ & -0.323381 & -3.433925 & -6.080872 \\
\hline $\mathrm{C}$ & 0.131336 & 5.288103 & -1.194845 & $\mathrm{C}$ & -0.094134 & -1.646591 & -4.494543 \\
\hline $\mathrm{H}$ & -0.646856 & 7.301520 & 1.167516 & $\mathrm{C}$ & -0.339313 & -1.132201 & -6.836218 \\
\hline $\mathrm{H}$ & -0.646856 & 7.301520 & -1.167516 & $\mathrm{C}$ & -0.401007 & -2.495127 & -7.107266 \\
\hline $\mathrm{C}$ & 0.106035 & 4.895804 & 2.561739 & $\mathrm{C}$ & -0.179529 & -3.015731 & -4.754897 \\
\hline $\mathrm{C}$ & 0.054571 & 4.197577 & 5.306246 & $\mathrm{H}$ & 0.067683 & -1.301622 & -3.479088 \\
\hline $\mathrm{C}$ & 0.039488 & 3.540672 & 2.970367 & $\mathrm{H}$ & -0.406332 & -0.405561 & -7.639252 \\
\hline $\mathrm{C}$ & 0.146945 & 5.881237 & 3.584105 & $\mathrm{H}$ & -0.521807 & -2.831079 & -8.132740 \\
\hline $\mathrm{C}$ & 0.121285 & 5.533036 & 4.923074 & $\mathrm{H}$ & -0.397913 & -4.493649 & -6.309184 \\
\hline $\mathrm{C}$ & 0.013790 & 3.193158 & 4.323391 & $\mathrm{C}$ & -0.099906 & -3.941069 & 3.598259 \\
\hline $\mathrm{H}$ & 0.007379 & 2.751621 & 2.225197 & $\mathrm{H}$ & -1.350437 & -2.707637 & 2.393175 \\
\hline $\mathrm{H}$ & 0.199175 & 6.930552 & 3.314229 & $\mathrm{C}$ & -0.710751 & -3.585407 & 2.428214 \\
\hline $\mathrm{H}$ & 0.153650 & 6.309605 & 5.681515 & $\mathrm{C}$ & 0.913883 & -5.856001 & 2.484367 \\
\hline $\mathrm{H}$ & 0.034289 & 3.917928 & 6.354018 & $\mathrm{C}$ & -0.457265 & -4.274996 & 1.210979 \\
\hline $\mathrm{C}$ & 0.106035 & 4.895804 & -2.561739 & $\mathrm{C}$ & 0.689629 & -5.136972 & 3.622218 \\
\hline $\mathrm{C}$ & 0.054571 & 4.197577 & -5.306246 & $\mathrm{C}$ & 0.402978 & -5.420073 & 1.218882 \\
\hline $\mathrm{C}$ & 0.146945 & 5.881237 & -3.584105 & $\mathrm{C}$ & -0.913840 & -3.762891 & 0.000000 \\
\hline $\mathrm{C}$ & 0.039488 & 3.540672 & -2.970367 & $\mathrm{H}$ & 1.152424 & -5.443332 & 4.556350 \\
\hline $\mathrm{C}$ & 0.013790 & 3.193158 & -4.323391 & $\mathrm{H}$ & 1.440263 & -6.858997 & 0.000000 \\
\hline $\mathrm{C}$ & 0.121285 & 5.533036 & -4.923074 & $\mathrm{H}$ & 1.542713 & -6.742024 & 2.514736 \\
\hline $\mathrm{H}$ & 0.199175 & 6.930552 & -3.314229 & $\mathrm{C}$ & -0.457265 & -4.274996 & -1.210979 \\
\hline $\mathrm{H}$ & 0.007379 & 2.751621 & -2.225197 & $\mathrm{H}$ & -1.550923 & -2.881299 & 0.000000 \\
\hline $\mathrm{H}$ & 0.153650 & 6.309605 & -5.681515 & $\mathrm{C}$ & -0.710751 & -3.585407 & -2.428214 \\
\hline $\mathrm{H}$ & 0.034289 & 3.917928 & -6.354018 & $\mathrm{C}$ & 0.402978 & -5.420073 & -1.218882 \\
\hline $\mathrm{C}$ & -0.054556 & 1.824714 & 4.740066 & $\mathrm{H}$ & 1.542713 & -6.742024 & -2.514736 \\
\hline
\end{tabular}




\begin{tabular}{cccccccc}
\hline & \multicolumn{3}{c}{ Cartesian Coordinates } & \multicolumn{4}{c}{ Cartesian Coordinates } \\
Atom & $\boldsymbol{X}$ & $\boldsymbol{Y}$ & $\boldsymbol{Z}$ & Atom & $\boldsymbol{X}$ & $\boldsymbol{Y}$ & $\boldsymbol{Z}$ \\
\hline $\mathrm{C}$ & -0.113073 & 0.688075 & 5.154376 & $\mathrm{C}$ & 0.777877 & -5.995875 & 0.000000 \\
$\mathrm{C}$ & -0.054556 & 1.824714 & -4.740066 & $\mathrm{C}$ & -0.099906 & -3.941069 & -3.598259 \\
$\mathrm{C}$ & -0.113073 & 0.688075 & -5.154376 & $\mathrm{H}$ & -1.350437 & -2.707637 & -2.393175 \\
$\mathrm{C}$ & -0.184603 & -0.695705 & 5.514119 & $\mathrm{C}$ & 0.689629 & -5.136972 & -3.622218 \\
$\mathrm{C}$ & -0.323381 & -3.433925 & 6.080872 & $\mathrm{H}$ & 1.152424 & -5.443332 & -4.556350 \\
$\mathrm{C}$ & -0.094134 & -1.646591 & 4.494543 & $\mathrm{C}$ & 0.913883 & -5.856001 & -2.484367 \\
$\mathrm{C}$ & -0.339313 & -1.132201 & 6.836218 & $\mathrm{H}$ & 1.104231 & 7.214988 & 1.168021 \\
$\mathrm{C}$ & -0.401007 & -2.495127 & 7.107266 & $\mathrm{H}$ & 1.104231 & 7.214988 & -1.168021 \\
$\mathrm{C}$ & -0.179529 & -3.015731 & 4.754897 & $\mathrm{C}$ & 0.090993 & 4.372126 & 0.000000 \\
$\mathrm{H}$ & 0.067683 & -1.301622 & 3.479088 & $\mathrm{H}$ & 0.936637 & 3.661119 & 0.000000 \\
$\mathrm{H}$ & -0.406332 & -0.405561 & 7.639252 & $\mathrm{H}$ & -0.813497 & 3.737882 & 0.000000 \\
$\mathrm{H}$ & -0.521807 & -2.831079 & 8.132740 & & & & \\
\hline
\end{tabular}




\section{DR3H1}

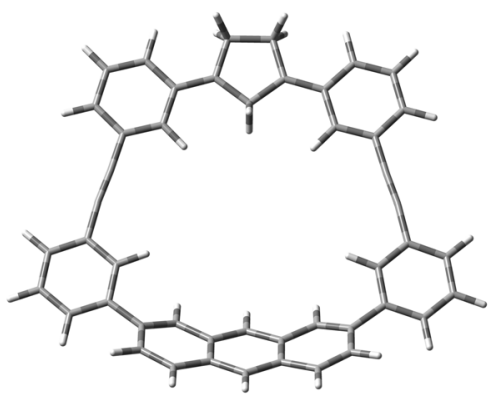

\#p opt freq um062x/6-31g(d)

Charge $=0$, Multiplicity $=3$, Point group $=C_{\mathrm{S}}$

Number of Imaginary Frequencies $=0$

Sum of electronic and zero-point Energies $=-1807.563823$ Hartree

Sum of electronic and thermal Energies $=-1807.528281$ Hartree

Sum of electronic and enthalpy Energies = -1807.527336 Hartree

Sum of electronic and thermal Free Energies $=-1807.633685$ Hartree

\begin{tabular}{|c|c|c|c|c|c|c|c|}
\hline \multirow[b]{2}{*}{ Atom } & \multicolumn{3}{|c|}{ Cartesian Coordinates } & \multicolumn{4}{|c|}{ Cartesian Coordinates } \\
\hline & $X$ & $Y$ & $Z$ & Atom & $X$ & $Y$ & $Z$ \\
\hline $\mathrm{C}$ & 0.118791 & 5.283197 & 1.192289 & $\mathrm{H}$ & -0.348310 & -4.496797 & 6.311469 \\
\hline $\mathrm{C}$ & 0.185118 & 6.724885 & 0.769418 & $\mathrm{C}$ & -0.165225 & -0.695254 & -5.522907 \\
\hline $\mathrm{C}$ & 0.185118 & 6.724885 & -0.769418 & $\mathrm{C}$ & -0.282247 & -3.435958 & -6.085937 \\
\hline $\mathrm{C}$ & 0.118791 & 5.283197 & -1.192289 & $\mathrm{C}$ & -0.094448 & -1.642933 & -4.498469 \\
\hline $\mathrm{H}$ & -0.665848 & 7.292605 & 1.169970 & $\mathrm{C}$ & -0.290771 & -1.135322 & -6.847777 \\
\hline $\mathrm{H}$ & -0.665848 & 7.292605 & -1.169970 & $\mathrm{C}$ & -0.342481 & -2.499427 & -7.116504 \\
\hline $\mathrm{C}$ & 0.097418 & 4.892782 & 2.562897 & $\mathrm{C}$ & -0.168613 & -3.013438 & -4.757537 \\
\hline $\mathrm{C}$ & 0.056523 & 4.204320 & 5.308029 & $\mathrm{H}$ & 0.046317 & -1.294452 & -3.481156 \\
\hline $\mathrm{C}$ & 0.035908 & 3.539903 & 2.973238 & $\mathrm{H}$ & -0.342001 & -0.409359 & -7.652244 \\
\hline $\mathrm{C}$ & 0.138142 & 5.882233 & 3.579298 & $\mathrm{H}$ & -0.440614 & -2.838220 & -8.143142 \\
\hline $\mathrm{C}$ & 0.117753 & 5.538602 & 4.920436 & $\mathrm{H}$ & -0.348310 & -4.496797 & -6.311469 \\
\hline $\mathrm{C}$ & 0.015475 & 3.197341 & 4.328041 & $\mathrm{C}$ & -0.105208 & -3.936680 & 3.599295 \\
\hline $\mathrm{H}$ & 0.003876 & 2.748546 & 2.230202 & $\mathrm{H}$ & -1.324481 & -2.673255 & 2.391679 \\
\hline $\mathrm{H}$ & 0.186199 & 6.929894 & 3.302117 & $\mathrm{C}$ & -0.704897 & -3.565363 & 2.427815 \\
\hline $\mathrm{H}$ & 0.150142 & 6.317847 & 5.675672 & $\mathrm{C}$ & 0.868230 & -5.874866 & 2.485792 \\
\hline $\mathrm{H}$ & 0.040396 & 3.925251 & 6.355704 & $\mathrm{C}$ & -0.466388 & -4.261423 & 1.210956 \\
\hline $\mathrm{C}$ & 0.097418 & 4.892782 & -2.562897 & $\mathrm{C}$ & 0.657469 & -5.152123 & 3.624294 \\
\hline $\mathrm{C}$ & 0.056523 & 4.204320 & -5.308029 & $\mathrm{C}$ & 0.368667 & -5.426900 & 1.219161 \\
\hline $\mathrm{C}$ & 0.138142 & 5.882233 & -3.579298 & $\mathrm{C}$ & -0.911637 & -3.738359 & 0.000000 \\
\hline $\mathrm{C}$ & 0.035908 & 3.539903 & -2.973238 & $\mathrm{H}$ & 1.111624 & -5.467230 & 4.559707 \\
\hline $\mathrm{C}$ & 0.015475 & 3.197341 & -4.328041 & $\mathrm{H}$ & 1.374229 & -6.888890 & 0.000000 \\
\hline $\mathrm{C}$ & 0.117753 & 5.538602 & -4.920436 & $\mathrm{H}$ & 1.477412 & -6.774470 & 2.514141 \\
\hline $\mathrm{H}$ & 0.186199 & 6.929894 & -3.302117 & $\mathrm{C}$ & -0.466388 & -4.261423 & -1.210956 \\
\hline $\mathrm{H}$ & 0.003876 & 2.748546 & -2.230202 & $\mathrm{H}$ & -1.528099 & -2.842143 & 0.000000 \\
\hline $\mathrm{H}$ & 0.150142 & 6.317847 & -5.675672 & $\mathrm{C}$ & -0.704897 & -3.565363 & -2.427815 \\
\hline $\mathrm{H}$ & 0.040396 & 3.925251 & -6.355704 & $\mathrm{C}$ & 0.368667 & -5.426900 & -1.219161 \\
\hline $\mathrm{C}$ & -0.047373 & 1.828512 & 4.750078 & $\mathrm{H}$ & 1.477412 & -6.774470 & -2.514141 \\
\hline
\end{tabular}




\begin{tabular}{cccccccc}
\hline & \multicolumn{3}{c}{ Cartesian Coordinates } & \multicolumn{4}{c}{ Cartesian Coordinates } \\
Atom & $\boldsymbol{X}$ & $\boldsymbol{Y}$ & $\boldsymbol{Z}$ & Atom & $\boldsymbol{X}$ & $\boldsymbol{Y}$ & $\boldsymbol{Z}$ \\
\hline $\mathrm{C}$ & -0.100531 & 0.690418 & 5.163398 & $\mathrm{C}$ & 0.731154 & -6.011170 & 0.000000 \\
$\mathrm{C}$ & -0.047373 & 1.828512 & -4.750078 & $\mathrm{C}$ & -0.105208 & -3.936680 & -3.599295 \\
$\mathrm{C}$ & -0.100531 & 0.690418 & -5.163398 & $\mathrm{H}$ & -1.324481 & -2.673255 & -2.391679 \\
$\mathrm{C}$ & -0.165225 & -0.695254 & 5.522907 & $\mathrm{C}$ & 0.657469 & -5.152123 & -3.624294 \\
$\mathrm{C}$ & -0.282247 & -3.435958 & 6.085937 & $\mathrm{H}$ & 1.111624 & -5.467230 & -4.559707 \\
$\mathrm{C}$ & -0.094448 & -1.642933 & 4.498469 & $\mathrm{C}$ & 0.868230 & -5.874866 & -2.485792 \\
$\mathrm{C}$ & -0.290771 & -1.135322 & 6.847777 & $\mathrm{H}$ & 1.084533 & 7.212020 & 1.170319 \\
$\mathrm{C}$ & -0.342481 & -2.499427 & 7.116504 & $\mathrm{H}$ & 1.084533 & 7.212020 & -1.170319 \\
$\mathrm{C}$ & -0.168613 & -3.013438 & 4.757537 & $\mathrm{C}$ & 0.079906 & 4.365007 & 0.000000 \\
$\mathrm{H}$ & 0.046317 & -1.294452 & 3.481156 & $\mathrm{H}$ & 0.926563 & 3.654750 & 0.000000 \\
$\mathrm{H}$ & -0.342001 & -0.409359 & 7.652244 & $\mathrm{H}$ & -0.823460 & 3.728571 & 0.000000 \\
$\mathrm{H}$ & -0.440614 & -2.838220 & 8.143142 & & & & \\
\hline
\end{tabular}




\section{DR3F1}

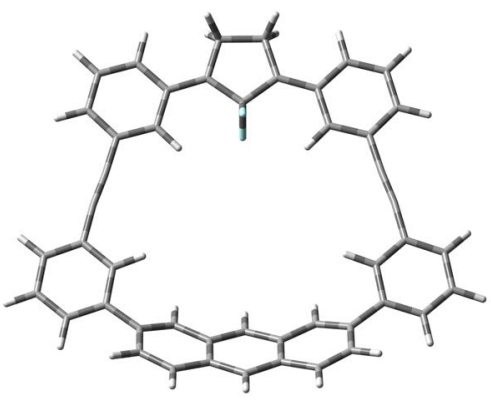

\#p opt freq ub3lyp/6-31g(d)

Charge $=0$, Multiplicity $=3$, Point group $=C_{\mathrm{S}}$

Number of Imaginary Frequencies $=1, v_{i}=-22.84$

Sum of electronic and zero-point Energies $=-2006.817829$ Hartree

Sum of electronic and thermal Energies = -2006.781219 Hartree

Sum of electronic and enthalpy Energies $=-2006.780275$ Hartree

Sum of electronic and thermal Free Energies $=-2006.887116$ Hartree

\begin{tabular}{|c|c|c|c|c|c|c|c|}
\hline \multirow[b]{2}{*}{ Atom } & \multicolumn{3}{|c|}{ Cartesian Coordinates } & \multicolumn{4}{|c|}{ Cartesian Coordinates } \\
\hline & $X$ & $Y$ & $Z$ & Atom & $X$ & $Y$ & $Z$ \\
\hline $\mathrm{C}$ & 0.075451 & 4.254462 & 0.000000 & $\mathrm{H}$ & -0.416460 & -3.005925 & 8.167317 \\
\hline $\mathrm{C}$ & 0.112916 & 5.153307 & 1.207526 & $\mathrm{H}$ & -0.339211 & -4.672103 & 6.342992 \\
\hline $\mathrm{C}$ & 0.173757 & 6.593571 & 0.774337 & $\mathrm{C}$ & -0.155322 & -0.863355 & -5.533114 \\
\hline $\mathrm{C}$ & 0.173757 & 6.593571 & -0.774337 & $\mathrm{C}$ & -0.272170 & -3.613089 & -6.108577 \\
\hline $\mathrm{C}$ & 0.112916 & 5.153307 & -1.207526 & $\mathrm{C}$ & -0.091547 & -1.822526 & -4.510372 \\
\hline $\mathrm{H}$ & -0.678809 & 7.163996 & 1.169488 & $\mathrm{C}$ & -0.272902 & -1.303715 & -6.865438 \\
\hline $\mathrm{H}$ & -0.678809 & 7.163996 & -1.169488 & $\mathrm{C}$ & -0.324457 & -2.669851 & -7.137987 \\
\hline $\mathrm{C}$ & 0.092180 & 4.737798 & 2.569311 & $\mathrm{C}$ & -0.165518 & -3.197550 & -4.771538 \\
\hline $\mathrm{C}$ & 0.050725 & 4.027266 & 5.314285 & $\mathrm{H}$ & 0.044015 & -1.476790 & -3.492116 \\
\hline $\mathrm{C}$ & 0.035982 & 3.373340 & 2.968994 & $\mathrm{H}$ & -0.317946 & -0.577216 & -7.670732 \\
\hline $\mathrm{C}$ & 0.127878 & 5.723788 & 3.598297 & $\mathrm{H}$ & -0.416460 & -3.005925 & -8.167317 \\
\hline $\mathrm{C}$ & 0.107107 & 5.368150 & 4.938553 & $\mathrm{H}$ & -0.339211 & -4.672103 & -6.342992 \\
\hline $\mathrm{C}$ & 0.015075 & 3.017004 & 4.325491 & $\mathrm{C}$ & -0.108299 & -4.125391 & 3.614600 \\
\hline $\mathrm{H}$ & 0.008659 & 2.595576 & 2.215507 & $\mathrm{H}$ & -1.315843 & -2.854010 & 2.392844 \\
\hline $\mathrm{H}$ & 0.171661 & 6.773864 & 3.330722 & $\mathrm{C}$ & -0.702748 & -3.749822 & 2.431786 \\
\hline $\mathrm{H}$ & 0.134971 & 6.141121 & 5.701815 & $\mathrm{C}$ & 0.846488 & -6.078405 & 2.492491 \\
\hline $\mathrm{H}$ & 0.034270 & 3.743323 & 6.361357 & $\mathrm{C}$ & -0.468748 & -4.445390 & 1.215736 \\
\hline $\mathrm{C}$ & 0.092180 & 4.737798 & -2.569311 & $\mathrm{C}$ & 0.639088 & -5.350751 & 3.636854 \\
\hline $\mathrm{C}$ & 0.050725 & 4.027266 & -5.314285 & $\mathrm{C}$ & 0.358016 & -5.627201 & 1.224090 \\
\hline $\mathrm{C}$ & 0.127878 & 5.723788 & -3.598297 & $\mathrm{C}$ & -0.910686 & -3.920168 & 0.000000 \\
\hline $\mathrm{C}$ & 0.035982 & 3.373340 & -2.968994 & $\mathrm{H}$ & 1.089821 & -5.676154 & 4.570633 \\
\hline $\mathrm{C}$ & 0.015075 & 3.017004 & -4.325491 & $\mathrm{H}$ & 1.344637 & -7.102171 & 0.000000 \\
\hline $\mathrm{C}$ & 0.107107 & 5.368150 & -4.938553 & $\mathrm{H}$ & 1.446570 & -6.984747 & 2.526870 \\
\hline $\mathrm{H}$ & 0.171661 & 6.773864 & -3.330722 & $\mathrm{C}$ & -0.468748 & -4.445390 & -1.215736 \\
\hline $\mathrm{H}$ & 0.008659 & 2.595576 & -2.215507 & $\mathrm{H}$ & -1.518548 & -3.017999 & 0.000000 \\
\hline $\mathrm{H}$ & 0.134971 & 6.141121 & -5.701815 & $\mathrm{C}$ & -0.702748 & -3.749822 & -2.431786 \\
\hline $\mathrm{H}$ & 0.034270 & 3.743323 & -6.361357 & $\mathrm{C}$ & 0.358016 & -5.627201 & -1.224090 \\
\hline
\end{tabular}




\begin{tabular}{cccccccc}
\hline & \multicolumn{3}{c}{ Cartesian Coordinates } & \multicolumn{4}{c}{ Cartesian Coordinates } \\
Atom & $\boldsymbol{X}$ & $\boldsymbol{Y}$ & $\boldsymbol{Z}$ & Atom & $\boldsymbol{X}$ & $\boldsymbol{Y}$ & $\boldsymbol{Z}$ \\
\hline $\mathrm{C}$ & -0.042676 & 1.653039 & 4.741735 & $\mathrm{H}$ & 1.446570 & -6.984747 & -2.526870 \\
$\mathrm{C}$ & -0.092902 & 0.515219 & 5.170220 & $\mathrm{C}$ & 0.712843 & -6.216000 & 0.000000 \\
$\mathrm{C}$ & -0.042676 & 1.653039 & -4.741735 & $\mathrm{C}$ & -0.108299 & -4.125391 & -3.614600 \\
$\mathrm{C}$ & -0.092902 & 0.515219 & -5.170220 & $\mathrm{H}$ & -1.315843 & -2.854010 & -2.392844 \\
$\mathrm{C}$ & -0.155322 & -0.863355 & 5.533114 & $\mathrm{C}$ & 0.639088 & -5.350751 & -3.636854 \\
$\mathrm{C}$ & -0.272170 & -3.613089 & 6.108577 & $\mathrm{H}$ & 1.089821 & -5.676154 & -4.570633 \\
$\mathrm{C}$ & -0.091547 & -1.822526 & 4.510372 & $\mathrm{C}$ & 0.846488 & -6.078405 & -2.492491 \\
$\mathrm{C}$ & -0.272902 & -1.303715 & 6.865438 & $\mathrm{~F}$ & 1.130166 & 3.348442 & 0.000000 \\
$\mathrm{C}$ & -0.324457 & -2.669851 & 7.137987 & $\mathrm{~F}$ & -1.052464 & 3.440786 & 0.000000 \\
$\mathrm{C}$ & -0.165518 & -3.197550 & 4.771538 & $\mathrm{H}$ & 1.071237 & 7.090125 & 1.169797 \\
$\mathrm{H}$ & 0.044015 & -1.476790 & 3.492116 & $\mathrm{H}$ & 1.071237 & 7.090125 & -1.169797 \\
$\mathrm{H}$ & -0.317946 & -0.577216 & 7.670732 & & & & \\
\hline
\end{tabular}




\section{DR3F1}

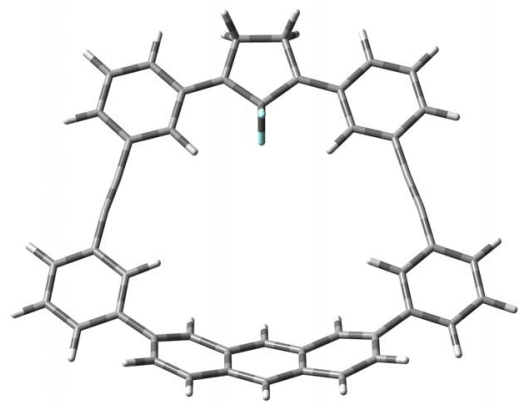

\#p opt freq uwb97xd/6-31g(d)

Charge $=0$, Multiplicity $=3$, Point group $=C_{\mathrm{S}}$

Number of Imaginary Frequencies $=1, v_{i}=-16.77$

Sum of electronic and zero-point Energies $=-2006.140359$ Hartree

Sum of electronic and thermal Energies = -2006.104205 Hartree

Sum of electronic and enthalpy Energies = -2006.103261 Hartree

Sum of electronic and thermal Free Energies $=-2006.209060$ Hartree

\begin{tabular}{|c|c|c|c|c|c|c|c|}
\hline \multicolumn{5}{|c|}{ Cartesian Coordinates } & \multicolumn{3}{|c|}{ Cartesian Coordinates } \\
\hline Atom & $X$ & $Y$ & $Z$ & Atom & $X$ & $Y$ & $Z$ \\
\hline $\mathrm{C}$ & -0.081772 & 4.233251 & 0.000000 & $\mathrm{H}$ & 0.516595 & -3.002921 & -8.132121 \\
\hline $\mathrm{C}$ & -0.123701 & 5.134921 & -1.202992 & $\mathrm{H}$ & 0.396059 & -4.666252 & -6.309231 \\
\hline $\mathrm{C}$ & -0.192953 & 6.570726 & -0.771443 & $\mathrm{C}$ & 0.181237 & -0.869845 & 5.511763 \\
\hline $\mathrm{C}$ & -0.192953 & 6.570726 & 0.771443 & $\mathrm{C}$ & 0.320943 & -3.606723 & 6.080205 \\
\hline $\mathrm{C}$ & -0.123701 & 5.134921 & 1.202992 & $\mathrm{C}$ & 0.092010 & -1.820811 & 4.492269 \\
\hline $\mathrm{H}$ & 0.657727 & 7.141302 & -1.167161 & $\mathrm{C}$ & 0.334665 & -1.304844 & 6.834426 \\
\hline $\mathrm{H}$ & 0.657727 & 7.141302 & 1.167161 & $\mathrm{C}$ & 0.396723 & -2.667510 & 7.106367 \\
\hline $\mathrm{C}$ & -0.100361 & 4.723205 & -2.564132 & $\mathrm{C}$ & 0.178243 & -3.189745 & 4.753697 \\
\hline $\mathrm{C}$ & -0.054693 & 4.019460 & -5.298940 & $\mathrm{H}$ & -0.068987 & -1.475998 & 3.476657 \\
\hline $\mathrm{C}$ & -0.035936 & 3.364497 & -2.962104 & $\mathrm{H}$ & 0.400674 & -0.577612 & 7.637027 \\
\hline $\mathrm{C}$ & -0.141997 & 5.707726 & -3.585444 & $\mathrm{H}$ & 0.516595 & -3.002921 & 8.132121 \\
\hline $\mathrm{C}$ & -0.119182 & 5.356469 & -4.923684 & $\mathrm{H}$ & 0.396059 & -4.666252 & 6.309231 \\
\hline $\mathrm{C}$ & -0.013134 & 3.016172 & -4.314013 & $\mathrm{C}$ & 0.101307 & -4.115961 & -3.597638 \\
\hline $\mathrm{H}$ & -0.003601 & 2.583601 & -2.211686 & $\mathrm{H}$ & 1.347893 & -2.878860 & -2.391817 \\
\hline $\mathrm{H}$ & -0.192375 & 6.757437 & -3.317011 & $\mathrm{C}$ & 0.711300 & -3.758792 & -2.427628 \\
\hline $\mathrm{H}$ & -0.151937 & 6.129904 & -5.684953 & $\mathrm{C}$ & -0.906055 & -6.034616 & -2.484375 \\
\hline $\mathrm{H}$ & -0.036533 & 3.735906 & -6.345791 & $\mathrm{C}$ & 0.460257 & -4.449769 & -1.210793 \\
\hline $\mathrm{C}$ & -0.100361 & 4.723205 & 2.564132 & $\mathrm{C}$ & -0.684451 & -5.314309 & -3.621958 \\
\hline $\mathrm{C}$ & -0.054693 & 4.019460 & 5.298940 & $\mathrm{C}$ & -0.396255 & -5.597615 & -1.218834 \\
\hline $\mathrm{C}$ & -0.141997 & 5.707726 & 3.585444 & $\mathrm{C}$ & 0.915257 & -3.935976 & 0.000000 \\
\hline $\mathrm{C}$ & -0.035936 & 3.364497 & 2.962104 & $\mathrm{H}$ & -1.146720 & -5.621681 & -4.556035 \\
\hline $\mathrm{C}$ & -0.013134 & 3.016172 & 4.314013 & $\mathrm{H}$ & -1.429046 & -7.039887 & 0.000000 \\
\hline $\mathrm{C}$ & -0.119182 & 5.356469 & 4.923684 & $\mathrm{H}$ & -1.532218 & -6.922528 & -2.514934 \\
\hline $\mathrm{H}$ & -0.192375 & 6.757437 & 3.317011 & $\mathrm{C}$ & 0.460257 & -4.449769 & 1.210793 \\
\hline $\mathrm{H}$ & -0.003601 & 2.583601 & 2.211686 & $\mathrm{H}$ & 1.548397 & -3.051622 & 0.000000 \\
\hline $\mathrm{H}$ & -0.151937 & 6.129904 & 5.684953 & $\mathrm{C}$ & 0.711300 & -3.758792 & 2.427628 \\
\hline $\mathrm{H}$ & -0.036533 & 3.735906 & 6.345791 & $\mathrm{C}$ & -0.396255 & -5.597615 & 1.218834 \\
\hline
\end{tabular}




\begin{tabular}{cccccccc}
\hline & \multicolumn{3}{c}{ Cartesian Coordinates } & \multicolumn{4}{c}{ Cartesian Coordinates } \\
Atom & $\boldsymbol{X}$ & $\boldsymbol{Y}$ & $\boldsymbol{Z}$ & Atom & $\boldsymbol{X}$ & $\boldsymbol{Y}$ & $\boldsymbol{Z}$ \\
\hline $\mathrm{C}$ & 0.053349 & 1.648315 & -4.731464 & $\mathrm{H}$ & -1.532218 & -6.922528 & 2.514934 \\
$\mathrm{C}$ & 0.110468 & 0.513486 & -5.150317 & $\mathrm{C}$ & -0.769230 & -6.174793 & 0.000000 \\
$\mathrm{C}$ & 0.053349 & 1.648315 & 4.731464 & $\mathrm{C}$ & 0.101307 & -4.115961 & 3.597638 \\
$\mathrm{C}$ & 0.110468 & 0.513486 & 5.150317 & $\mathrm{H}$ & 1.347893 & -2.878860 & 2.391817 \\
$\mathrm{C}$ & 0.181237 & -0.869845 & -5.511763 & $\mathrm{C}$ & -0.684451 & -5.314309 & 3.621958 \\
$\mathrm{C}$ & 0.320943 & -3.606723 & -6.080205 & $\mathrm{H}$ & -1.146720 & -5.621681 & 4.556035 \\
$\mathrm{C}$ & 0.092010 & -1.820811 & -4.492269 & $\mathrm{C}$ & -0.906055 & -6.034616 & 2.484375 \\
$\mathrm{C}$ & 0.334665 & -1.304844 & -6.834426 & $\mathrm{~F}$ & -1.127293 & 3.334795 & 0.000000 \\
$\mathrm{C}$ & 0.396723 & -2.667510 & -7.106367 & $\mathrm{~F}$ & 1.043882 & 3.436879 & 0.000000 \\
$\mathrm{C}$ & 0.178243 & -3.189745 & -4.753697 & $\mathrm{H}$ & -1.094383 & 7.056849 & -1.167577 \\
$\mathrm{H}$ & -0.068987 & -1.475998 & -3.476657 & $\mathrm{H}$ & -1.094383 & 7.056849 & 1.167577 \\
$\mathrm{H}$ & 0.400674 & -0.577612 & -7.637027 & & & & \\
\hline
\end{tabular}




\section{DR3F1}

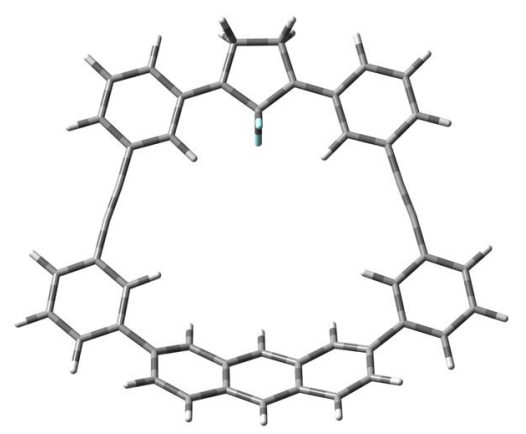

\#p opt freq um062x/6-31g(d)

Charge $=0$, Multiplicity $=3$, Point group $=C_{\mathrm{S}}$

Number of Imaginary Frequencies $=1, v_{i}=-11.05$

Sum of electronic and zero-point Energies $=-2005.998307$ Hartree

Sum of electronic and thermal Energies = -2005.962036 Hartree

Sum of electronic and enthalpy Energies $=-2005.961092$ Hartree

Sum of electronic and thermal Free Energies $=-2006.066982$ Hartree

\begin{tabular}{|c|c|c|c|c|c|c|c|}
\hline \multicolumn{5}{|c|}{ Cartesian Coordinates } & \multicolumn{3}{|c|}{ Cartesian Coordinates } \\
\hline Atom & $X$ & $Y$ & $Z$ & Atom & $X$ & $Y$ & $Z$ \\
\hline $\mathrm{C}$ & -0.072033 & 4.254905 & 0.000000 & $\mathrm{H}$ & 0.416392 & -2.993640 & -8.133866 \\
\hline $\mathrm{C}$ & -0.110293 & 5.159085 & -1.199788 & $\mathrm{H}$ & 0.335878 & -4.665648 & -6.313948 \\
\hline $\mathrm{C}$ & -0.172266 & 6.595451 & -0.772304 & $\mathrm{C}$ & 0.156981 & -0.870951 & 5.495774 \\
\hline $\mathrm{C}$ & -0.172266 & 6.595451 & 0.772304 & $\mathrm{C}$ & 0.271200 & -3.606516 & 6.080050 \\
\hline $\mathrm{C}$ & -0.110293 & 5.159085 & 1.199788 & $\mathrm{C}$ & 0.092715 & -1.825658 & 4.477678 \\
\hline $\mathrm{H}$ & 0.680740 & 7.160441 & -1.170367 & $\mathrm{C}$ & 0.274457 & -1.300468 & 6.824807 \\
\hline $\mathrm{H}$ & 0.680740 & 7.160441 & 1.170367 & $\mathrm{C}$ & 0.324719 & -2.662462 & 7.104159 \\
\hline $\mathrm{C}$ & -0.091226 & 4.738381 & -2.560654 & $\mathrm{C}$ & 0.166021 & -3.194239 & 4.747754 \\
\hline $\mathrm{C}$ & -0.056425 & 4.015453 & -5.285493 & $\mathrm{H}$ & -0.042124 & -1.484415 & 3.457140 \\
\hline $\mathrm{C}$ & -0.033564 & 3.376871 & -2.942826 & $\mathrm{H}$ & 0.320518 & -0.568431 & 7.624079 \\
\hline $\mathrm{C}$ & -0.131016 & 5.716808 & -3.584988 & $\mathrm{H}$ & 0.416392 & -2.993640 & 8.133866 \\
\hline $\mathrm{C}$ & -0.113689 & 5.355765 & -4.921642 & $\mathrm{H}$ & 0.335878 & -4.665648 & 6.313948 \\
\hline $\mathrm{C}$ & -0.016116 & 3.019234 & -4.293029 & $\mathrm{C}$ & 0.109826 & -4.125424 & -3.595563 \\
\hline $\mathrm{H}$ & -0.002611 & 2.604319 & -2.182412 & $\mathrm{H}$ & 1.337215 & -2.869361 & -2.388150 \\
\hline $\mathrm{H}$ & -0.175822 & 6.767516 & -3.318566 & $\mathrm{C}$ & 0.716630 & -3.760672 & -2.425762 \\
\hline $\mathrm{H}$ & -0.145198 & 6.123020 & -5.688743 & $\mathrm{C}$ & -0.861640 & -6.066661 & -2.485603 \\
\hline $\mathrm{H}$ & -0.042506 & 3.720805 & -6.329075 & $\mathrm{C}$ & 0.481084 & -4.460415 & -1.210596 \\
\hline $\mathrm{C}$ & -0.091226 & 4.738381 & 2.560654 & $\mathrm{C}$ & -0.654443 & -5.339835 & -3.622258 \\
\hline $\mathrm{C}$ & -0.056425 & 4.015453 & 5.285493 & $\mathrm{C}$ & -0.357380 & -5.623398 & -1.219089 \\
\hline $\mathrm{C}$ & -0.131016 & 5.716808 & 3.584988 & $\mathrm{C}$ & 0.929192 & -3.939250 & 0.000000 \\
\hline $\mathrm{C}$ & -0.033564 & 3.376871 & 2.942826 & $\mathrm{H}$ & -1.113556 & -5.650485 & -4.556776 \\
\hline $\mathrm{C}$ & -0.016116 & 3.019234 & 4.293029 & $\mathrm{H}$ & -1.366695 & -7.083032 & 0.000000 \\
\hline $\mathrm{C}$ & -0.113689 & 5.355765 & 4.921642 & $\mathrm{H}$ & -1.472856 & -6.964858 & -2.514906 \\
\hline $\mathrm{H}$ & -0.175822 & 6.767516 & 3.318566 & $\mathrm{C}$ & 0.481084 & -4.460415 & 1.210596 \\
\hline $\mathrm{H}$ & -0.002611 & 2.604319 & 2.182412 & $\mathrm{H}$ & 1.546510 & -3.043685 & 0.000000 \\
\hline $\mathrm{H}$ & -0.145198 & 6.123020 & 5.688743 & $\mathrm{C}$ & 0.716630 & -3.760672 & 2.425762 \\
\hline $\mathrm{H}$ & -0.042506 & 3.720805 & 6.329075 & $\mathrm{C}$ & -0.357380 & -5.623398 & 1.219089 \\
\hline
\end{tabular}




\begin{tabular}{cccccccc}
\hline & \multicolumn{3}{c}{ Cartesian Coordinates } & \multicolumn{4}{c}{ Cartesian Coordinates } \\
Atom & $\boldsymbol{X}$ & $\boldsymbol{Y}$ & $\boldsymbol{Z}$ & Atom & $\boldsymbol{X}$ & $\boldsymbol{Y}$ & $\boldsymbol{Z}$ \\
\hline $\mathrm{C}$ & 0.043378 & 1.648084 & -4.707000 & $\mathrm{H}$ & -1.472856 & -6.964858 & 2.514906 \\
$\mathrm{C}$ & 0.094054 & 0.512224 & -5.126284 & $\mathrm{C}$ & -0.720549 & -6.207566 & 0.000000 \\
$\mathrm{C}$ & 0.043378 & 1.648084 & 4.707000 & $\mathrm{C}$ & 0.109826 & -4.125424 & 3.595563 \\
$\mathrm{C}$ & 0.094054 & 0.512224 & 5.126284 & $\mathrm{H}$ & 1.337215 & -2.869361 & 2.388150 \\
$\mathrm{C}$ & 0.156981 & -0.870951 & -5.495774 & $\mathrm{C}$ & -0.654443 & -5.339835 & 3.622258 \\
$\mathrm{C}$ & 0.271200 & -3.606516 & -6.080050 & $\mathrm{H}$ & -1.113556 & -5.650485 & 4.556776 \\
$\mathrm{C}$ & 0.092715 & -1.825658 & -4.477678 & $\mathrm{C}$ & -0.861640 & -6.066661 & 2.485603 \\
$\mathrm{C}$ & 0.274457 & -1.300468 & -6.824807 & $\mathrm{~F}$ & -1.117007 & 3.362031 & 0.000000 \\
$\mathrm{C}$ & 0.324719 & -2.662462 & -7.104159 & $\mathrm{~F}$ & 1.045219 & 3.453794 & 0.000000 \\
$\mathrm{C}$ & 0.166021 & -3.194239 & -4.747754 & $\mathrm{H}$ & -1.070804 & 7.084676 & -1.170543 \\
$\mathrm{H}$ & -0.042124 & -1.484415 & -3.457140 & $\mathrm{H}$ & -1.070804 & 7.084676 & 1.170543 \\
$\mathrm{H}$ & 0.320518 & -0.568431 & -7.624079 & & & & \\
\hline
\end{tabular}




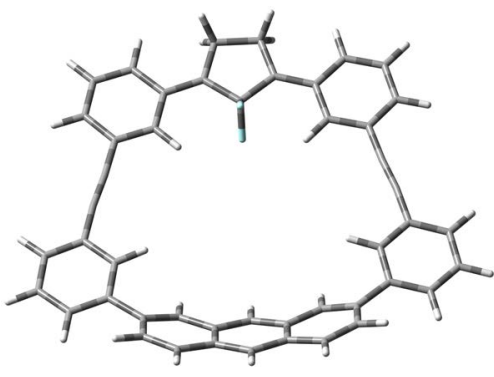

\#p opt freq ub3lyp/6-31g(d)

Charge $=1$, Multiplicity $=2$, Point group $=C_{1}$

Number of Imaginary Frequencies $=0$

Sum of electronic and zero-point Energies $=-2006.599063$ Hartree

Sum of electronic and thermal Energies $=-2006.561579$ Hartree

Sum of electronic and enthalpy Energies $=-2006.560635$ Hartree

Sum of electronic and thermal Free Energies $=-2006.671665$ Hartree

\begin{tabular}{|c|c|c|c|c|c|c|c|}
\hline \multirow[b]{2}{*}{ Atom } & \multicolumn{3}{|c|}{ Cartesian Coordinates } & \multirow[b]{2}{*}{ Atom } & \multicolumn{3}{|c|}{ Cartesian Coordinates } \\
\hline & $X$ & $Y$ & $Z$ & & $X$ & $Y$ & $Z$ \\
\hline $\mathrm{C}$ & -4.267035 & -0.018205 & 0.076978 & $\mathrm{H}$ & 3.021007 & -8.143804 & -0.401867 \\
\hline $\mathrm{C}$ & -5.167372 & -1.225339 & 0.122888 & $\mathrm{H}$ & 4.692278 & -6.326598 & -0.331770 \\
\hline $\mathrm{C}$ & -6.602504 & -0.799815 & 0.193982 & $\mathrm{C}$ & 0.839423 & 5.507499 & -0.144317 \\
\hline $\mathrm{C}$ & -6.611361 & 0.741013 & 0.156806 & $\mathrm{C}$ & 3.579148 & 6.118885 & -0.234727 \\
\hline $\mathrm{C}$ & -5.179667 & 1.180264 & 0.103720 & $\mathrm{C}$ & 1.807532 & 4.493231 & -0.095529 \\
\hline $\mathrm{H}$ & -7.182834 & -1.221700 & -0.637383 & $\mathrm{C}$ & 1.261799 & 6.847288 & -0.238020 \\
\hline $\mathrm{H}$ & -7.168167 & 1.112759 & -0.714401 & $\mathrm{C}$ & 2.624642 & 7.137773 & -0.278147 \\
\hline C & -4.738101 & -2.575159 & 0.105843 & $\mathrm{C}$ & 3.179206 & 4.775043 & -0.153311 \\
\hline $\mathrm{C}$ & -3.995634 & -5.293497 & 0.073709 & $\mathrm{H}$ & 1.472422 & 3.468884 & 0.019846 \\
\hline $\mathrm{C}$ & -3.364095 & -2.950158 & 0.040467 & $\mathrm{H}$ & 0.526493 & 7.644570 & -0.274069 \\
\hline $\mathrm{C}$ & -5.719151 & -3.612470 & 0.155502 & $\mathrm{H}$ & 2.948654 & 8.171623 & -0.352335 \\
\hline $\mathrm{C}$ & -5.345723 & -4.944233 & 0.139102 & $\mathrm{H}$ & 4.635013 & 6.368467 & -0.290281 \\
\hline $\mathrm{C}$ & -2.988236 & -4.299151 & 0.023956 & $\mathrm{C}$ & 4.154718 & -3.595077 & -0.117489 \\
\hline $\mathrm{H}$ & -2.598867 & -2.185125 & 0.002504 & $\mathrm{H}$ & 2.896964 & -2.377387 & -1.352368 \\
\hline $\mathrm{H}$ & -6.771346 & -3.357282 & 0.206949 & $\mathrm{C}$ & 3.782800 & -2.409536 & -0.725130 \\
\hline $\mathrm{H}$ & -6.102366 & -5.721631 & 0.177242 & $\mathrm{C}$ & 6.099699 & -2.464078 & 0.840917 \\
\hline $\mathrm{H}$ & -3.700364 & -6.337513 & 0.060708 & $\mathrm{C}$ & 4.474452 & -1.197693 & -0.487727 \\
\hline $\mathrm{C}$ & -4.764968 & 2.533973 & 0.076200 & $\mathrm{C}$ & 5.371856 & -3.614212 & 0.632092 \\
\hline $\mathrm{C}$ & -4.048577 & 5.259086 & 0.018326 & $\mathrm{C}$ & 5.648144 & -1.202395 & 0.347915 \\
\hline $\mathrm{C}$ & -5.757029 & 3.561895 & 0.095415 & $\mathrm{C}$ & 3.948256 & 0.018858 & -0.935153 \\
\hline $\mathrm{C}$ & -3.393483 & 2.922071 & 0.027841 & $\mathrm{H}$ & 5.697283 & -4.543930 & 1.089756 \\
\hline $\mathrm{C}$ & -3.030534 & 4.274042 & -0.000968 & $\mathrm{H}$ & 7.112611 & 0.028233 & 1.345302 \\
\hline $\mathrm{C}$ & -5.395889 & 4.896925 & 0.066369 & $\mathrm{H}$ & 7.001963 & -2.497938 & 1.445946 \\
\hline $\mathrm{H}$ & -6.807402 & 3.296928 & 0.131829 & $\mathrm{C}$ & 4.462670 & 1.238107 & -0.481587 \\
\hline $\mathrm{H}$ & -2.620302 & 2.164204 & 0.013948 & $\mathrm{H}$ & 3.058294 & 0.016118 & -1.560208 \\
\hline $\mathrm{H}$ & -6.160610 & 5.667218 & 0.080809 & $\mathrm{C}$ & 3.759754 & 2.444585 & -0.713560 \\
\hline $\mathrm{H}$ & -3.763403 & 6.305715 & -0.004225 & $\mathrm{C}$ & 5.636067 & 1.249737 & 0.354364 \\
\hline
\end{tabular}




\begin{tabular}{cccccccc}
\hline & \multicolumn{3}{c}{ Cartesian Coordinates } & \multicolumn{4}{c}{ Cartesian Coordinates } \\
Atom & $\boldsymbol{X}$ & $\boldsymbol{Y}$ & $\boldsymbol{Z}$ & Atom & $\boldsymbol{X}$ & $\boldsymbol{Y}$ & $\boldsymbol{Z}$ \\
\hline $\mathrm{C}$ & -1.624949 & -4.706369 & -0.042117 & $\mathrm{H}$ & 6.976609 & 2.552515 & 1.459993 \\
$\mathrm{C}$ & -0.486497 & -5.131751 & -0.096976 & $\mathrm{C}$ & 6.229575 & 0.025544 & 0.710183 \\
$\mathrm{C}$ & -1.669700 & 4.692049 & -0.048971 & $\mathrm{C}$ & 4.120025 & 3.630594 & -0.100030 \\
$\mathrm{C}$ & -0.534457 & 5.127202 & -0.091322 & $\mathrm{H}$ & 2.874835 & 2.406866 & -1.341743 \\
$\mathrm{C}$ & 0.890267 & -5.499191 & -0.161446 & $\mathrm{C}$ & 5.336288 & 3.657201 & 0.650751 \\
$\mathrm{C}$ & 3.634537 & -6.086748 & -0.269315 & $\mathrm{H}$ & 5.652739 & 4.587619 & 1.113235 \\
$\mathrm{C}$ & 1.849863 & -4.477192 & -0.108451 & $\mathrm{C}$ & 6.075051 & 2.513021 & 0.854254 \\
$\mathrm{C}$ & 1.323649 & -6.834680 & -0.268628 & $\mathrm{~F}$ & -3.372120 & -0.006156 & 1.133585 \\
$\mathrm{C}$ & 2.688628 & -7.113423 & -0.317347 & $\mathrm{~F}$ & -3.460424 & -0.022456 & -1.048843 \\
$\mathrm{C}$ & 3.223456 & -4.746957 & -0.174722 & $\mathrm{H}$ & -7.078276 & -1.176652 & 1.109913 \\
$\mathrm{H}$ & 1.506896 & -3.456698 & 0.017580 & $\mathrm{H}$ & -7.121352 & 1.158487 & 1.035284 \\
$\mathrm{H}$ & 0.594876 & -7.637770 & -0.308405 & & & & \\
\hline
\end{tabular}




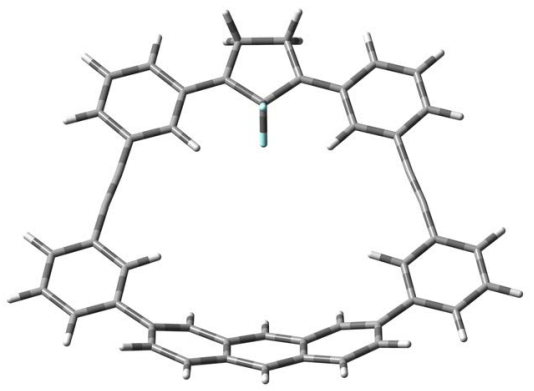

\#p opt freq ub3lyp/6-31g(d)

Charge $=2$, Multiplicity $=3$, Point group $=C_{1}$

Number of Imaginary Frequencies $=0$

Sum of electronic and zero-point Energies $=-2006.299286$ Hartree

Sum of electronic and thermal Energies = -2006.261767 Hartree

Sum of electronic and enthalpy Energies = -2006.260823 Hartree

Sum of electronic and thermal Free Energies $=-2006.371110$ Hartree

\begin{tabular}{|c|c|c|c|c|c|c|c|}
\hline \multicolumn{5}{|c|}{ Cartesian Coordinates } & \multicolumn{3}{|c|}{ Cartesian Coordinates } \\
\hline Atom & $\boldsymbol{X}$ & $Y$ & $Z$ & Atom & $X$ & $Y$ & $Z$ \\
\hline $\mathrm{C}$ & 4.297405 & -0.000643 & 0.066314 & $\mathrm{H}$ & -2.961761 & 8.147549 & -0.221091 \\
\hline $\mathrm{C}$ & 5.207354 & 1.199645 & 0.095636 & $\mathrm{H}$ & -4.659008 & 6.356274 & -0.210838 \\
\hline $\mathrm{C}$ & 6.637960 & 0.768213 & 0.146499 & $\mathrm{C}$ & -0.865458 & -5.462495 & -0.116319 \\
\hline $\mathrm{C}$ & 6.637704 & -0.770254 & 0.146687 & $\mathrm{C}$ & -3.606985 & -6.096496 & -0.169771 \\
\hline $\mathrm{C}$ & 5.206957 & -1.201226 & 0.095916 & $\mathrm{C}$ & -1.836891 & -4.452046 & -0.110782 \\
\hline $\mathrm{H}$ & 7.199743 & 1.166110 & -0.709390 & $\mathrm{C}$ & -1.285758 & -6.807014 & -0.159181 \\
\hline $\mathrm{H}$ & 7.199387 & -1.168538 & -0.709080 & $\mathrm{C}$ & -2.647508 & -7.109182 & -0.184799 \\
\hline $\mathrm{C}$ & 4.777393 & 2.546616 & 0.077484 & $\mathrm{C}$ & -3.208634 & -4.747681 & -0.143371 \\
\hline $\mathrm{C}$ & 4.024147 & 5.256002 & 0.042666 & $\mathrm{H}$ & -1.502601 & -3.423537 & -0.035904 \\
\hline $\mathrm{C}$ & 3.398457 & 2.913228 & 0.031348 & $\mathrm{H}$ & -0.547803 & -7.602335 & -0.168288 \\
\hline $\mathrm{C}$ & 5.757855 & 3.587085 & 0.105830 & $\mathrm{H}$ & -2.964153 & -8.146688 & -0.221817 \\
\hline $\mathrm{C}$ & 5.378536 & 4.916373 & 0.088303 & $\mathrm{H}$ & -4.660997 & -6.355027 & -0.211506 \\
\hline $\mathrm{C}$ & 3.016680 & 4.258252 & 0.013746 & $\mathrm{C}$ & -4.153639 & 3.616169 & -0.110901 \\
\hline $\mathrm{H}$ & 2.637349 & 2.143871 & 0.010337 & $\mathrm{H}$ & -2.903604 & 2.389057 & -1.365791 \\
\hline $\mathrm{H}$ & 6.811801 & 3.336709 & 0.141124 & $\mathrm{C}$ & -3.788029 & 2.418050 & -0.737771 \\
\hline $\mathrm{H}$ & 6.130056 & 5.698787 & 0.109882 & $\mathrm{C}$ & -6.130924 & 2.485165 & 0.785263 \\
\hline $\mathrm{H}$ & 3.725888 & 6.299342 & 0.028994 & $\mathrm{C}$ & -4.495625 & 1.223858 & -0.520850 \\
\hline $\mathrm{C}$ & 4.776546 & -2.548053 & 0.077996 & $\mathrm{C}$ & -5.380895 & 3.638470 & 0.601261 \\
\hline $\mathrm{C}$ & 4.022358 & -5.257184 & 0.043635 & $\mathrm{C}$ & -5.681613 & 1.231353 & 0.293220 \\
\hline $\mathrm{C}$ & 5.756636 & -3.588861 & 0.106879 & $\mathrm{C}$ & -3.971182 & 0.000584 & -0.972666 \\
\hline $\mathrm{C}$ & 3.397496 & -2.914193 & 0.031545 & $\mathrm{H}$ & -5.710536 & 4.562488 & 1.065940 \\
\hline $\mathrm{C}$ & 3.015250 & -4.259087 & 0.014150 & $\mathrm{H}$ & -7.175749 & 0.001042 & 1.254124 \\
\hline $\mathrm{C}$ & 5.376852 & -4.918019 & 0.089601 & $\mathrm{H}$ & -7.043955 & 2.528602 & 1.372541 \\
\hline $\mathrm{H}$ & 6.810661 & -3.338851 & 0.142426 & $\mathrm{C}$ & -4.496065 & -1.222547 & -0.520986 \\
\hline $\mathrm{H}$ & 2.636666 & -2.144574 & 0.010092 & $\mathrm{H}$ & -3.084028 & 0.000460 & -1.601116 \\
\hline $\mathrm{H}$ & 6.128089 & -5.700692 & 0.111636 & $\mathrm{C}$ & -3.788882 & -2.416971 & -0.738006 \\
\hline $\mathrm{H}$ & 3.723733 & -6.300421 & 0.030148 & $\mathrm{C}$ & -5.682063 & -1.229704 & 0.293071 \\
\hline
\end{tabular}




\begin{tabular}{cccccccc}
\hline & \multicolumn{3}{c}{ Cartesian Coordinates } & \multicolumn{4}{c}{ Cartesian Coordinates } \\
Atom & $\boldsymbol{X}$ & $\boldsymbol{Y}$ & $\boldsymbol{Z}$ & Atom & $\boldsymbol{X}$ & $\boldsymbol{Y}$ & $\boldsymbol{Z}$ \\
\hline $\mathrm{C}$ & 1.650482 & 4.660124 & -0.032180 & $\mathrm{H}$ & -7.044876 & -2.526564 & 1.372260 \\
$\mathrm{C}$ & 0.510521 & 5.082227 & -0.070630 & $\mathrm{C}$ & -6.277792 & 0.000919 & 0.640603 \\
$\mathrm{C}$ & 1.648919 & -4.660471 & -0.032090 & $\mathrm{C}$ & -4.154949 & -3.615016 & -0.111281 \\
$\mathrm{C}$ & 0.508803 & -5.082142 & -0.070708 & $\mathrm{H}$ & -2.904387 & -2.388227 & -1.365943 \\
$\mathrm{C}$ & -0.863665 & 5.462876 & -0.116030 & $\mathrm{C}$ & -5.382214 & -3.636957 & 0.600869 \\
$\mathrm{C}$ & -3.605052 & 6.097498 & -0.169214 & $\mathrm{H}$ & -5.712171 & -4.560898 & 1.065478 \\
$\mathrm{C}$ & -1.835327 & 4.452643 & -0.110510 & $\mathrm{C}$ & -6.131834 & -2.483398 & 0.784979 \\
$\mathrm{C}$ & -1.283666 & 6.807490 & -0.158725 & $\mathrm{~F}$ & 3.412272 & -0.000377 & 1.129597 \\
$\mathrm{C}$ & -2.645351 & 7.109967 & -0.184205 & $\mathrm{~F}$ & 3.483402 & -0.000642 & -1.052739 \\
$\mathrm{C}$ & -3.207016 & 4.748581 & -0.142992 & $\mathrm{H}$ & 7.137317 & 1.166682 & 1.040050 \\
$\mathrm{H}$ & -1.501245 & 3.424064 & -0.035726 & $\mathrm{H}$ & 7.136890 & -1.168670 & 1.040362 \\
$\mathrm{H}$ & -0.545536 & 7.602649 & -0.167820 & & & & \\
\hline
\end{tabular}




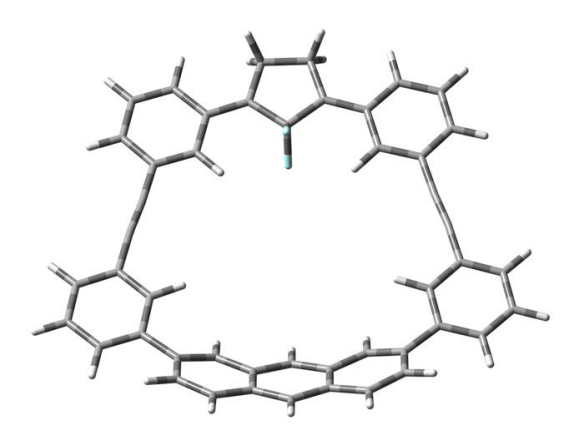

\#p opt freq ub3lyp/6-31g(d) guess=(mix, always $)$

Charge $=2$, Multiplicity $=1$, Point group $=C_{1}$

Number of Imaginary Frequencies $=0$

Sum of electronic and zero-point Energies $=-2006.299302$ Hartree

Sum of electronic and thermal Energies = -2006.261783 Hartree

Sum of electronic and enthalpy Energies = -2006.260839 Hartree

Sum of electronic and thermal Free Energies $=-2006.370081$ Hartree

\begin{tabular}{|c|c|c|c|c|c|c|c|}
\hline \multirow[b]{2}{*}{ Atom } & \multicolumn{3}{|c|}{ Cartesian Coordinates } & \multicolumn{4}{|c|}{ Cartesian Coordinates } \\
\hline & $X$ & $Y$ & $Z$ & Atom & $X$ & $Y$ & $Z$ \\
\hline $\mathrm{C}$ & 4.297401 & 0.000459 & 0.065897 & $\mathrm{H}$ & -2.963898 & 8.146897 & -0.219011 \\
\hline $\mathrm{C}$ & 5.207023 & 1.200998 & 0.095280 & $\mathrm{H}$ & -4.660774 & 6.355273 & -0.209744 \\
\hline $\mathrm{C}$ & 6.637755 & 0.769961 & 0.145830 & $\mathrm{C}$ & -0.863899 & -5.462909 & -0.115531 \\
\hline $\mathrm{C}$ & 6.637923 & -0.768516 & 0.145980 & $\mathrm{C}$ & -3.605372 & -6.097386 & -0.168756 \\
\hline $\mathrm{C}$ & 5.207289 & -1.199877 & 0.095427 & $\mathrm{C}$ & -1.835509 & -4.452605 & -0.110621 \\
\hline $\mathrm{H}$ & 7.199251 & 1.168095 & -0.710135 & $\mathrm{C}$ & -1.283997 & -6.807509 & -0.157709 \\
\hline $\mathrm{H}$ & 7.199553 & -1.166709 & -0.709868 & $\mathrm{C}$ & -2.645720 & -7.109896 & -0.183240 \\
\hline $\mathrm{C}$ & 4.776671 & 2.547848 & 0.077345 & $\mathrm{C}$ & -3.207231 & -4.748488 & -0.143040 \\
\hline $\mathrm{C}$ & 4.022651 & 5.257019 & 0.042905 & $\mathrm{H}$ & -1.501359 & -3.424009 & -0.036341 \\
\hline $\mathrm{C}$ & 3.397629 & 2.914064 & 0.031322 & $\mathrm{H}$ & -0.545921 & -7.602720 & -0.166347 \\
\hline $\mathrm{C}$ & 5.756845 & 3.588603 & 0.105773 & $\mathrm{H}$ & -2.962197 & -8.147472 & -0.219734 \\
\hline $\mathrm{C}$ & 5.377150 & 4.917783 & 0.088439 & $\mathrm{H}$ & -4.659342 & -6.356107 & -0.210317 \\
\hline $\mathrm{C}$ & 3.015462 & 4.258987 & 0.013900 & $\mathrm{C}$ & -4.154666 & 3.615194 & -0.111115 \\
\hline $\mathrm{H}$ & 2.636730 & 2.144506 & 0.010273 & $\mathrm{H}$ & -2.903464 & 2.388326 & -1.365112 \\
\hline $\mathrm{H}$ & 6.810868 & 3.338535 & 0.141003 & $\mathrm{C}$ & -3.788306 & 2.417138 & -0.737661 \\
\hline $\mathrm{H}$ & 6.128457 & 5.700398 & 0.110110 & $\mathrm{C}$ & -6.132092 & 2.483628 & 0.784025 \\
\hline $\mathrm{H}$ & 3.724081 & 6.300272 & 0.029379 & $\mathrm{C}$ & -4.495708 & 1.222765 & -0.521137 \\
\hline $\mathrm{C}$ & 4.777247 & -2.546829 & 0.077691 & $\mathrm{C}$ & -5.382334 & 3.637155 & 0.600373 \\
\hline $\mathrm{C}$ & 4.023886 & -5.256188 & 0.043625 & $\mathrm{C}$ & -5.682152 & 1.229927 & 0.292266 \\
\hline $\mathrm{C}$ & 5.757668 & -3.587342 & 0.106466 & $\mathrm{C}$ & -3.970606 & -0.000357 & -0.972604 \\
\hline $\mathrm{C}$ & 3.398298 & -2.913387 & 0.031531 & $\mathrm{H}$ & -5.712528 & 4.561117 & 1.064763 \\
\hline $\mathrm{C}$ & 3.016460 & -4.258407 & 0.014281 & $\mathrm{H}$ & -7.176387 & -0.000816 & 1.252453 \\
\hline $\mathrm{C}$ & 5.378296 & -4.916615 & 0.089320 & $\mathrm{H}$ & -7.045453 & 2.526793 & 1.370812 \\
\hline $\mathrm{H}$ & 6.811624 & -3.337012 & 0.141825 & $\mathrm{C}$ & -4.495381 & -1.223631 & -0.521173 \\
\hline $\mathrm{H}$ & 2.637215 & -2.144019 & 0.010198 & $\mathrm{H}$ & -3.083105 & -0.000229 & -1.600567 \\
\hline $\mathrm{H}$ & 6.129789 & -5.699046 & 0.111263 & $\mathrm{C}$ & -3.787672 & -2.417816 & -0.737746 \\
\hline $\mathrm{H}$ & 3.725572 & -6.299515 & 0.030232 & $\mathrm{C}$ & -5.681819 & -1.231130 & 0.292234 \\
\hline
\end{tabular}




\begin{tabular}{cccccccc}
\hline & \multicolumn{3}{c}{ Cartesian Coordinates } & \multicolumn{3}{c}{ Cartesian Coordinates } \\
Atom & $\boldsymbol{X}$ & \multicolumn{1}{c}{$\boldsymbol{Y}$} & $\boldsymbol{Z}$ & Atom & $\boldsymbol{X}$ & $\boldsymbol{Y}$ & $\boldsymbol{Z}$ \\
\hline $\mathrm{C}$ & 1.649158 & 4.660459 & -0.031935 & $\mathrm{H}$ & -7.044793 & -2.528389 & 1.370717 \\
$\mathrm{C}$ & 0.509043 & 5.082183 & -0.070197 & $\mathrm{C}$ & -6.278125 & -0.000684 & 0.639381 \\
$\mathrm{C}$ & 1.650265 & -4.660233 & -0.031723 & $\mathrm{C}$ & -4.153712 & -3.615987 & -0.111226 \\
$\mathrm{C}$ & 0.510280 & -5.082293 & -0.070123 & $\mathrm{H}$ & -2.902874 & -2.388758 & -1.365242 \\
$\mathrm{C}$ & -0.865187 & 5.462629 & -0.115420 & $\mathrm{C}$ & -5.381382 & -3.638290 & 0.600244 \\
$\mathrm{C}$ & -3.606762 & 6.096707 & -0.168299 & $\mathrm{H}$ & -5.711362 & -4.562366 & 1.064561 \\
$\mathrm{C}$ & -1.836644 & 4.452182 & -0.110582 & $\mathrm{C}$ & -6.131435 & -2.484962 & 0.783947 \\
$\mathrm{C}$ & -1.285490 & 6.807174 & -0.157339 & $\mathrm{~F}$ & 3.412222 & 0.000415 & 1.129107 \\
$\mathrm{C}$ & -2.647263 & 7.109362 & -0.182708 & $\mathrm{~F}$ & 3.483386 & 0.000317 & -1.053136 \\
$\mathrm{C}$ & -3.208406 & 4.747865 & -0.142811 & $\mathrm{H}$ & 7.137170 & 1.168605 & 1.039270 \\
$\mathrm{H}$ & -1.502338 & 3.423617 & -0.036520 & $\mathrm{H}$ & 7.137375 & -1.166863 & 1.039534 \\
$\mathrm{H}$ & -0.547535 & 7.602498 & -0.165910 & & & & \\
\hline
\end{tabular}




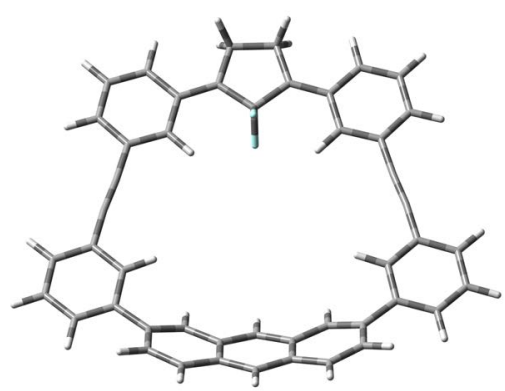

\#p opt freq rb3lyp/6-31g(d)

Charge $=2$, Multiplicity $=1$, Point group $=C_{1}$

Number of Imaginary Frequencies $=0$

Sum of electronic and zero-point Energies $=-2006.279205$ Hartree

Sum of electronic and thermal Energies $=-2006.241679$ Hartree

Sum of electronic and enthalpy Energies $=-2006.240735$ Hartree

Sum of electronic and thermal Free Energies $=-2006.350062$ Hartree

\begin{tabular}{|c|c|c|c|c|c|c|c|}
\hline \multirow[b]{2}{*}{ Atom } & \multicolumn{3}{|c|}{ Cartesian Coordinates } & \multirow[b]{2}{*}{ Atom } & \multicolumn{3}{|c|}{ Cartesian Coordinates } \\
\hline & $X$ & $Y$ & $Z$ & & $X$ & $Y$ & $Z$ \\
\hline $\mathrm{C}$ & 4.291153 & 0.000041 & 0.072489 & $\mathrm{H}$ & -2.968136 & 8.148147 & -0.315192 \\
\hline $\mathrm{C}$ & 5.200879 & 1.202635 & 0.105542 & $\mathrm{H}$ & -4.657840 & 6.349966 & -0.262553 \\
\hline $\mathrm{C}$ & 6.631044 & 0.769617 & 0.163395 & $\mathrm{C}$ & -0.864055 & -5.471913 & -0.138933 \\
\hline $\mathrm{C}$ & 6.631055 & -0.769493 & 0.163464 & $\mathrm{C}$ & -3.602788 & -6.096669 & -0.211768 \\
\hline $\mathrm{C}$ & 5.200899 & -1.202537 & 0.105574 & $\mathrm{C}$ & -1.832181 & -4.458591 & -0.102564 \\
\hline $\mathrm{H}$ & 7.198865 & 1.165412 & -0.689611 & $\mathrm{C}$ & -1.285401 & -6.816085 & -0.218120 \\
\hline $\mathrm{H}$ & 7.198934 & -1.165363 & -0.689469 & $\mathrm{C}$ & -2.647302 & -7.113283 & -0.251192 \\
\hline $\mathrm{C}$ & 4.767256 & 2.543061 & 0.084566 & $\mathrm{C}$ & -3.203138 & -4.748248 & -0.147775 \\
\hline $\mathrm{C}$ & 4.019041 & 5.253404 & 0.045654 & $\mathrm{H}$ & -1.496885 & -3.433172 & 0.003100 \\
\hline $\mathrm{C}$ & 3.387833 & 2.911517 & 0.032716 & $\mathrm{H}$ & -0.548529 & -7.611796 & -0.250542 \\
\hline $\mathrm{C}$ & 5.751220 & 3.586949 & 0.116296 & $\mathrm{H}$ & -2.967998 & -8.148201 & -0.315171 \\
\hline $\mathrm{C}$ & 5.375064 & 4.915075 & 0.096652 & $\mathrm{H}$ & -4.657722 & -6.350040 & -0.262473 \\
\hline $\mathrm{C}$ & 3.008020 & 4.256065 & 0.012895 & $\mathrm{C}$ & -4.148686 & 3.613393 & -0.101612 \\
\hline $\mathrm{H}$ & 2.626194 & 2.142968 & 0.008887 & $\mathrm{H}$ & -2.901987 & 2.387163 & -1.353959 \\
\hline $\mathrm{H}$ & 6.804516 & 3.334439 & 0.155772 & $\mathrm{C}$ & -3.784395 & 2.418597 & -0.722715 \\
\hline $\mathrm{H}$ & 6.126137 & 5.697725 & 0.120805 & $\mathrm{C}$ & -6.118374 & 2.486227 & 0.814878 \\
\hline $\mathrm{H}$ & 3.720861 & 6.296905 & 0.030425 & $\mathrm{C}$ & -4.490904 & 1.219987 & -0.500151 \\
\hline $\mathrm{C}$ & 4.767301 & -2.542972 & 0.084610 & $\mathrm{C}$ & -5.372623 & 3.637206 & 0.623118 \\
\hline $\mathrm{C}$ & 4.019144 & -5.253330 & 0.045716 & $\mathrm{C}$ & -5.671836 & 1.228301 & 0.322373 \\
\hline $\mathrm{C}$ & 5.751284 & -3.586839 & 0.116423 & $\mathrm{C}$ & -3.967330 & -0.000035 & -0.951674 \\
\hline $\mathrm{C}$ & 3.387889 & -2.911457 & 0.032692 & $\mathrm{H}$ & -5.699542 & 4.563114 & 1.086285 \\
\hline $\mathrm{C}$ & 3.008104 & -4.256013 & 0.012879 & $\mathrm{H}$ & -7.158494 & -0.000064 & 1.294749 \\
\hline $\mathrm{C}$ & 5.375157 & -4.914972 & 0.096788 & $\mathrm{H}$ & -7.027513 & 2.529268 & 1.408157 \\
\hline $\mathrm{H}$ & 6.804573 & -3.334306 & 0.155957 & $\mathrm{C}$ & -4.490875 & -1.220066 & -0.500142 \\
\hline $\mathrm{H}$ & 2.626235 & -2.142924 & 0.008791 & $\mathrm{H}$ & -3.080759 & -0.000027 & -1.581051 \\
\hline $\mathrm{H}$ & 6.126245 & -5.697607 & 0.121002 & $\mathrm{C}$ & -3.784342 & -2.418664 & -0.722705 \\
\hline $\mathrm{H}$ & 3.720987 & -6.296838 & 0.030485 & $\mathrm{C}$ & -5.671802 & -1.228402 & 0.322389 \\
\hline
\end{tabular}




\begin{tabular}{cccccccc}
\hline & \multicolumn{3}{c}{ Cartesian Coordinates } & \multicolumn{4}{c}{ Cartesian Coordinates } \\
Atom & $\boldsymbol{X}$ & $\boldsymbol{Y}$ & $\boldsymbol{Z}$ & Atom & $\boldsymbol{X}$ & $\boldsymbol{Y}$ & $\boldsymbol{Z}$ \\
\hline $\mathrm{C}$ & 1.647695 & 4.668150 & -0.040232 & $\mathrm{H}$ & -7.027438 & -2.529391 & 1.408198 \\
$\mathrm{C}$ & 0.508985 & 5.095692 & -0.085843 & $\mathrm{C}$ & -6.265414 & -0.000056 & 0.674417 \\
$\mathrm{C}$ & 1.647792 & -4.668130 & -0.040342 & $\mathrm{C}$ & -4.148598 & -3.613461 & -0.101586 \\
$\mathrm{C}$ & 0.509099 & -5.095706 & -0.086024 & $\mathrm{H}$ & -2.901944 & -2.387216 & -1.353962 \\
$\mathrm{C}$ & -0.864168 & 5.471884 & -0.138847 & $\mathrm{C}$ & -5.372525 & -3.637295 & 0.623161 \\
$\mathrm{C}$ & -3.602906 & 6.096607 & -0.211804 & $\mathrm{H}$ & -5.699420 & -4.563208 & 1.086334 \\
$\mathrm{C}$ & -1.832284 & 4.458551 & -0.102517 & $\mathrm{C}$ & -6.118304 & -2.486332 & 0.814914 \\
$\mathrm{C}$ & -1.285527 & 6.816051 & -0.218063 & $\mathrm{~F}$ & 3.403058 & 0.000044 & 1.132310 \\
$\mathrm{C}$ & -2.647431 & 7.113232 & -0.251197 & $\mathrm{~F}$ & 3.482935 & 0.000024 & -1.049750 \\
$\mathrm{C}$ & -3.203241 & 4.748191 & -0.147784 & $\mathrm{H}$ & 7.127748 & 1.165901 & 1.059538 \\
$\mathrm{H}$ & -1.496980 & 3.433137 & 0.003177 & $\mathrm{H}$ & 7.127709 & -1.165685 & 1.059676 \\
$\mathrm{H}$ & -0.548663 & 7.611771 & -0.250450 & & & & \\
\hline
\end{tabular}




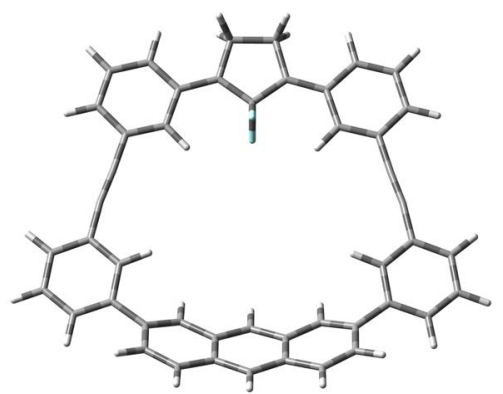

\#p opt freq ub3lyp/6-31+g(d)

Charge $=-1$, Multiplicity $=2$, Point group $=C_{1}$

Number of imaginary frequencies $=0$

Sum of electronic and zero-point Energies $=-2006.980765$ Hartree

Sum of electronic and thermal Energies $=-2006.943186$ Hartree

Sum of electronic and thermal Enthalpies $=-2006.942242$ Hartree

Sum of electronic and thermal Free Energies $=-2007.051467$ Hartree

\begin{tabular}{|c|c|c|c|c|c|c|c|}
\hline \multirow[b]{2}{*}{ Atoms } & \multicolumn{3}{|c|}{ Cartesian Coordinates } & \multirow[b]{2}{*}{ Atoms } & \multicolumn{3}{|c|}{ Cartesian Coordinates } \\
\hline & $X$ & $\boldsymbol{Y}$ & $Z$ & & $\boldsymbol{X}$ & $\boldsymbol{Y}$ & $Z$ \\
\hline $\mathrm{C}$ & 4.231231 & 0.000143 & 0.074433 & $\mathrm{C}$ & 5.101102 & 1.176499 & 0.107489 \\
\hline $\mathrm{C}$ & 6.548097 & 0.770406 & 0.159932 & $\mathrm{C}$ & 6.548152 & -0.769948 & 0.159983 \\
\hline $\mathrm{C}$ & 5.101187 & -1.176149 & 0.107544 & $\mathrm{H}$ & 7.121541 & 1.163361 & -0.697856 \\
\hline $\mathrm{H}$ & 7.121640 & -1.162923 & -0.697767 & $\mathrm{C}$ & 4.710132 & 2.549138 & 0.091838 \\
\hline $\mathrm{C}$ & 4.056467 & 5.346019 & 0.057672 & $\mathrm{C}$ & 3.355887 & 2.997953 & 0.043128 \\
\hline $\mathrm{C}$ & 5.709431 & 3.572767 & 0.124921 & $\mathrm{C}$ & 5.384723 & 4.925990 & 0.108136 \\
\hline $\mathrm{C}$ & 3.034594 & 4.363859 & 0.025593 & $\mathrm{H}$ & 2.558191 & 2.264329 & 0.017941 \\
\hline $\mathrm{H}$ & 6.756429 & 3.290053 & 0.163709 & $\mathrm{H}$ & 6.182904 & 5.666314 & 0.134405 \\
\hline $\mathrm{H}$ & 3.794508 & 6.399703 & 0.043606 & $\mathrm{C}$ & 4.710316 & -2.548817 & 0.091961 \\
\hline $\mathrm{C}$ & 4.056854 & -5.345747 & 0.057937 & $\mathrm{C}$ & 5.709689 & -3.572371 & 0.125093 \\
\hline $\mathrm{C}$ & 3.356103 & -2.997732 & 0.043279 & $\mathrm{C}$ & 3.034910 & -4.363662 & 0.025815 \\
\hline $\mathrm{C}$ & 5.385079 & -4.925619 & 0.108374 & $\mathrm{H}$ & 6.756667 & -3.289579 & 0.163866 \\
\hline $\mathrm{H}$ & 2.558354 & -2.264167 & 0.018066 & $\mathrm{H}$ & 6.183314 & -5.665884 & 0.134680 \\
\hline $\mathrm{H}$ & 3.794970 & -6.399450 & 0.043932 & $\mathrm{C}$ & 1.673821 & 4.798627 & -0.028304 \\
\hline $\mathrm{C}$ & 0.533813 & 5.230364 & -0.078510 & $\mathrm{C}$ & 1.674166 & -4.798532 & -0.028014 \\
\hline $\mathrm{C}$ & 0.534189 & -5.230354 & -0.078183 & $\mathrm{C}$ & -0.847562 & 5.576574 & -0.147830 \\
\hline $\mathrm{C}$ & -3.615307 & 6.121917 & -0.294043 & $\mathrm{C}$ & -1.802553 & 4.545844 & -0.075901 \\
\hline $\mathrm{C}$ & -1.309141 & 6.902648 & -0.285327 & $\mathrm{C}$ & -2.679617 & 7.160324 & -0.351300 \\
\hline $\mathrm{C}$ & -3.180430 & 4.791141 & -0.165418 & $\mathrm{H}$ & -1.444661 & 3.533502 & 0.075976 \\
\hline $\mathrm{H}$ & -0.591528 & 7.716433 & -0.336904 & $\mathrm{H}$ & -3.024919 & 8.186095 & -0.460584 \\
\hline $\mathrm{H}$ & -4.676691 & 6.343843 & -0.375838 & $\mathrm{C}$ & -0.847156 & -5.576637 & -0.147736 \\
\hline $\mathrm{C}$ & -3.614852 & -6.122139 & -0.294281 & $\mathrm{C}$ & -1.802218 & -4.545969 & -0.075852 \\
\hline $\mathrm{C}$ & -1.308639 & -6.902731 & -0.285368 & $\mathrm{C}$ & -2.679092 & -7.160485 & -0.351505 \\
\hline $\mathrm{C}$ & -3.180070 & -4.791343 & -0.165533 & $\mathrm{H}$ & -1.444402 & -3.533620 & 0.076152 \\
\hline $\mathrm{H}$ & -0.590971 & -7.716471 & -0.336905 & $\mathrm{H}$ & -3.024320 & -8.186271 & -0.460883 \\
\hline $\mathrm{H}$ & -4.676214 & -6.344127 & -0.376193 & $\mathrm{C}$ & -4.097606 & 3.623998 & -0.105497 \\
\hline $\mathrm{H}$ & -2.802853 & 2.395746 & -1.283169 & $\mathrm{C}$ & -3.708818 & 2.436475 & -0.684952 \\
\hline
\end{tabular}




\begin{tabular}{cccc|cccc}
\hline & \multicolumn{3}{c}{ Cartesian Coordinates } & \multicolumn{4}{c}{ Cartesian Coordinates } \\
Atoms & $\boldsymbol{X}$ & $\boldsymbol{Y}$ & $\boldsymbol{Z}$ & Atoms & $\boldsymbol{X}$ & $\boldsymbol{Y}$ & $\boldsymbol{Z}$ \\
\hline $\mathrm{C}$ & -6.059026 & 2.495283 & 0.835288 & $\mathrm{C}$ & -4.403929 & 1.217598 & -0.452883 \\
$\mathrm{C}$ & -5.332428 & 3.643005 & 0.628705 & $\mathrm{C}$ & -5.598358 & 1.225243 & 0.356855 \\
$\mathrm{C}$ & -3.868773 & -0.000114 & -0.882959 & $\mathrm{H}$ & -5.667798 & 4.577632 & 1.071702 \\
$\mathrm{H}$ & -7.084777 & -0.000269 & 1.328240 & $\mathrm{H}$ & -6.971813 & 2.530099 & 1.427073 \\
$\mathrm{C}$ & -4.403835 & -1.217879 & -0.452918 & $\mathrm{H}$ & -2.954132 & -0.000070 & -1.472329 \\
$\mathrm{C}$ & -3.708625 & -2.436696 & -0.685011 & $\mathrm{C}$ & -5.598271 & -1.225639 & 0.356810 \\
$\mathrm{H}$ & -6.971649 & -2.530635 & 1.426956 & $\mathrm{C}$ & -6.190649 & -0.000226 & 0.706202 \\
$\mathrm{C}$ & -4.097333 & -3.624267 & -0.105599 & $\mathrm{H}$ & -2.802657 & -2.395877 & -1.283216 \\
$\mathrm{C}$ & -5.332173 & -3.643393 & 0.628568 & $\mathrm{H}$ & -5.667483 & -4.578060 & 1.071526 \\
$\mathrm{C}$ & -6.058855 & -2.495731 & 0.835186 & $\mathrm{~F}$ & 3.219105 & 0.000132 & 1.129405 \\
$\mathrm{~F}$ & 3.303620 & 0.000083 & -1.057938 & $\mathrm{H}$ & 7.057993 & 1.163662 & 1.056796 \\
$\mathrm{H}$ & 7.058060 & -1.163105 & 1.056884 & & & & \\
\hline
\end{tabular}




\section{DR3HF1}

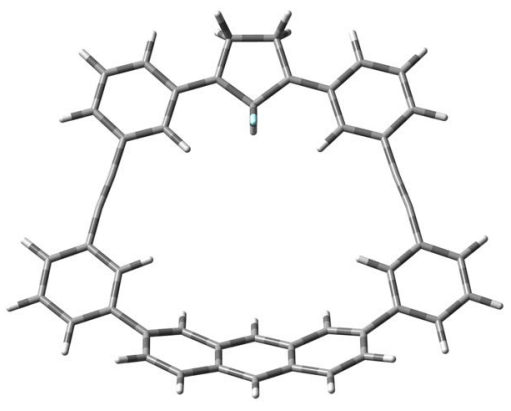

\#p opt freq ub3lyp/6-31g(d)

Charge $=0$, Multiplicity $=3$, Point group $=C_{\mathrm{S}}$

Number of imaginary frequencies $=1, v_{i}=-12.06$

Sum of electronic and zero-point Energies $=-1907.566055$ Hartree

Sum of electronic and thermal Energies $=-1907.530351$ Hartree

Sum of electronic and thermal Enthalpies $=-1907.529407$ Hartree

Sum of electronic and thermal Free Energies = -1907.634016 Hartree

\begin{tabular}{|c|c|c|c|c|c|c|c|}
\hline \multirow[b]{2}{*}{ Atoms } & \multicolumn{3}{|c|}{ Cartesian Coordinates } & \multirow[b]{2}{*}{ Atoms } & \multicolumn{3}{|c|}{ Cartesian Coordinates } \\
\hline & $X$ & $Y$ & $Z$ & & $X$ & $Y$ & $Z$ \\
\hline $\mathrm{C}$ & 4.335392 & -0.000007 & -0.074150 & $\mathrm{C}$ & 5.231305 & 1.200678 & 0.080762 \\
\hline $\mathrm{C}$ & 6.672160 & 0.773045 & 0.196527 & $\mathrm{C}$ & 6.672154 & -0.773057 & 0.196587 \\
\hline $\mathrm{C}$ & 5.231303 & -1.200690 & 0.080788 & $\mathrm{H}$ & 7.276455 & 1.169142 & -0.632697 \\
\hline $\mathrm{H}$ & 7.276492 & -1.169226 & -0.632572 & $\mathrm{C}$ & 4.825594 & 2.567719 & 0.058350 \\
\hline $\mathrm{C}$ & 4.120721 & 5.316274 & -0.012864 & $\mathrm{C}$ & 3.462888 & 2.972293 & 0.010179 \\
\hline $\mathrm{C}$ & 5.812688 & 3.595505 & 0.089201 & $\mathrm{C}$ & 5.460060 & 4.936692 & 0.051615 \\
\hline $\mathrm{C}$ & 3.108550 & 4.329427 & -0.030576 & $\mathrm{H}$ & 2.682472 & 2.220311 & 0.031457 \\
\hline $\mathrm{H}$ & 6.862489 & 3.326926 & 0.134641 & $\mathrm{H}$ & 6.235326 & 5.698044 & 0.070638 \\
\hline $\mathrm{H}$ & 3.839736 & 6.363833 & -0.043444 & $\mathrm{C}$ & 4.825588 & -2.567729 & 0.058394 \\
\hline $\mathrm{C}$ & 4.120708 & -5.316284 & -0.012787 & $\mathrm{C}$ & 5.812678 & -3.595518 & 0.089290 \\
\hline $\mathrm{C}$ & 3.462882 & -2.972301 & 0.010198 & $\mathrm{C}$ & 3.108540 & -4.329434 & -0.030541 \\
\hline $\mathrm{C}$ & 5.460047 & -4.936705 & 0.051719 & $\mathrm{H}$ & 6.862479 & -3.326942 & 0.134753 \\
\hline $\mathrm{H}$ & 2.682468 & -2.220316 & 0.031444 & $\mathrm{H}$ & 6.235309 & -5.698059 & 0.070777 \\
\hline $\mathrm{H}$ & 3.839721 & -6.363842 & -0.043354 & $\mathrm{C}$ & 1.744385 & 4.746377 & -0.081759 \\
\hline $\mathrm{C}$ & 0.605816 & 5.173712 & -0.126762 & $\mathrm{C}$ & 1.744375 & -4.746380 & -0.081747 \\
\hline $\mathrm{C}$ & 0.605805 & -5.173712 & -0.126764 & $\mathrm{C}$ & -0.773366 & 5.535442 & -0.181651 \\
\hline $\mathrm{C}$ & -3.524618 & 6.109085 & -0.282621 & $\mathrm{C}$ & -1.731808 & 4.512529 & -0.108626 \\
\hline $\mathrm{C}$ & -1.215596 & 6.867092 & -0.300521 & $\mathrm{C}$ & -2.582206 & 7.138750 & -0.344036 \\
\hline $\mathrm{C}$ & -3.107410 & 4.772683 & -0.174702 & $\mathrm{H}$ & -1.384848 & 3.494844 & 0.028314 \\
\hline $\mathrm{H}$ & -0.489920 & 7.672716 & -0.352475 & $\mathrm{H}$ & -2.919369 & 8.167663 & -0.436824 \\
\hline $\mathrm{H}$ & -4.584188 & 6.342650 & -0.343586 & $\mathrm{C}$ & -0.773376 & -5.535439 & -0.181674 \\
\hline $\mathrm{C}$ & -3.524628 & -6.109075 & -0.282674 & $\mathrm{C}$ & -1.731816 & -4.512525 & -0.108648 \\
\hline $\mathrm{C}$ & -1.215608 & -6.867088 & -0.300561 & $\mathrm{C}$ & -2.582219 & -7.138741 & -0.344092 \\
\hline $\mathrm{C}$ & -3.107419 & -4.772676 & -0.174738 & $\mathrm{H}$ & -1.384856 & -3.494842 & 0.028306 \\
\hline $\mathrm{H}$ & -0.489934 & -7.672712 & -0.352516 & $\mathrm{H}$ & -2.919384 & -8.167653 & -0.436893 \\
\hline $\mathrm{H}$ & -4.584198 & -6.342638 & -0.343650 & $\mathrm{C}$ & -4.034042 & 3.615186 & -0.109286 \\
\hline $\mathrm{H}$ & -2.770351 & 2.393312 & -1.324430 & $\mathrm{C}$ & -3.662007 & 2.432054 & -0.705267 \\
\hline
\end{tabular}




\begin{tabular}{cccc|cccc}
\hline & \multicolumn{3}{c}{ Cartesian Coordinates } & \multicolumn{4}{c}{ Cartesian Coordinates } \\
Atoms & $\boldsymbol{X}$ & $\boldsymbol{Y}$ & $\boldsymbol{Z}$ & Atoms & $\boldsymbol{X}$ & $\boldsymbol{Y}$ & $\boldsymbol{Z}$ \\
\hline $\mathrm{C}$ & -5.979662 & 2.492658 & 0.860034 & $\mathrm{C}$ & -4.355345 & 1.215811 & -0.465798 \\
$\mathrm{C}$ & -5.254124 & 3.637287 & 0.646696 & $\mathrm{C}$ & -5.531434 & 1.224147 & 0.369075 \\
$\mathrm{C}$ & -3.833198 & 0.000007 & -0.911097 & $\mathrm{H}$ & -5.576819 & 4.571248 & 1.098982 \\
$\mathrm{H}$ & -6.999392 & 0.000001 & 1.365991 & $\mathrm{H}$ & -6.881769 & 2.526991 & 1.466459 \\
$\mathrm{C}$ & -4.355347 & -1.215799 & -0.465809 & $\mathrm{H}$ & -2.935270 & 0.000009 & -1.525258 \\
$\mathrm{C}$ & -3.662012 & -2.432041 & -0.705286 & $\mathrm{C}$ & -5.531437 & -1.224140 & 0.369064 \\
$\mathrm{H}$ & -6.881776 & -2.526990 & 1.466434 & $\mathrm{C}$ & -6.117593 & 0.000003 & 0.728116 \\
$\mathrm{C}$ & -4.034049 & -3.615178 & -0.109315 & $\mathrm{H}$ & -2.770354 & -2.393296 & -1.324448 \\
$\mathrm{C}$ & -5.254132 & -3.637283 & 0.646663 & $\mathrm{H}$ & -5.576830 & -4.571247 & 1.098941 \\
$\mathrm{C}$ & -5.979669 & -2.492654 & 0.860011 & $\mathrm{~F}$ & 3.273216 & 0.000004 & 0.874994 \\
$\mathrm{H}$ & 7.131452 & 1.172388 & 1.112111 & $\mathrm{H}$ & 7.131395 & -1.172330 & 1.112228 \\
$\mathrm{H}$ & 3.817416 & -0.000016 & -1.047778 & & & & \\
\hline
\end{tabular}




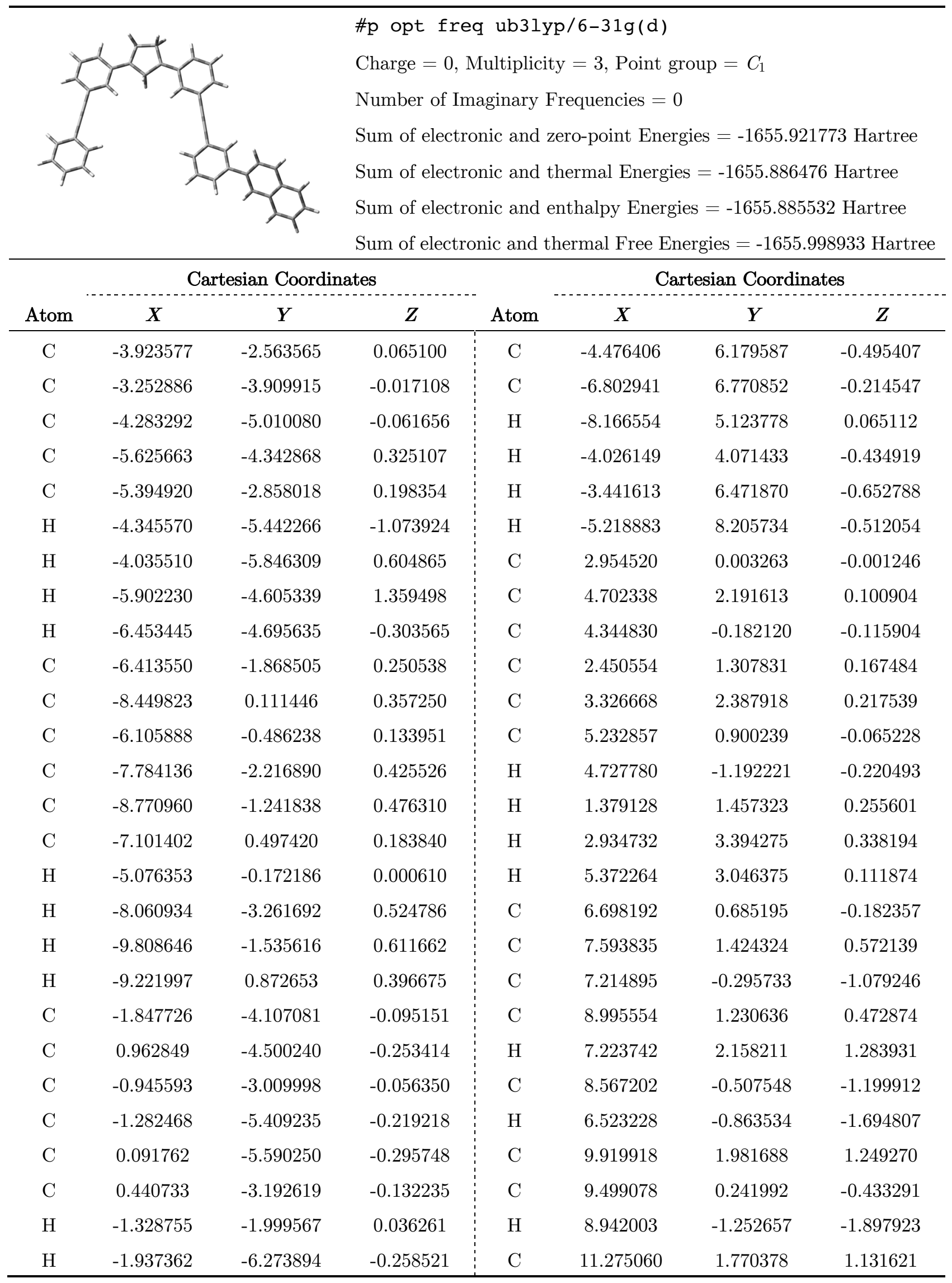




\begin{tabular}{cccccccc}
\hline & \multicolumn{3}{c}{ Cartesian Coordinates } & \multicolumn{3}{c}{ Cartesian Coordinates } \\
Atom & $\boldsymbol{X}$ & $\boldsymbol{Y}$ & $\boldsymbol{Z}$ & Atom & $\boldsymbol{X}$ & $\boldsymbol{Y}$ & $\boldsymbol{Z}$ \\
\hline $\mathrm{H}$ & 0.495887 & -6.594760 & -0.390823 & $\mathrm{H}$ & 9.536751 & 2.729702 & 1.939564 \\
$\mathrm{H}$ & 2.036814 & -4.642862 & -0.312482 & $\mathrm{C}$ & 10.902368 & 0.048912 & -0.532104 \\
$\mathrm{C}$ & -6.758369 & 1.876344 & 0.060420 & $\mathrm{C}$ & 11.771859 & 0.795109 & 0.231689 \\
$\mathrm{C}$ & -6.472145 & 3.053829 & -0.045172 & $\mathrm{H}$ & 11.970768 & 2.352378 & 1.730073 \\
$\mathrm{C}$ & 1.318630 & -2.069321 & -0.087034 & $\mathrm{H}$ & 11.279985 & -0.701352 & -1.223054 \\
$\mathrm{C}$ & 2.072472 & -1.115631 & -0.047728 & $\mathrm{H}$ & 12.843802 & 0.637870 & 0.148056 \\
$\mathrm{C}$ & -6.136280 & 4.433240 & -0.169730 & $\mathrm{H}$ & -3.546986 & -1.965978 & 0.916539 \\
$\mathrm{C}$ & -5.474596 & 7.154021 & -0.416530 & $\mathrm{H}$ & -3.705460 & -1.942671 & -0.824128 \\
$\mathrm{C}$ & -7.135252 & 5.424601 & -0.091821 & $\mathrm{H}$ & -7.583461 & 7.524447 & -0.152469 \\
$\mathrm{C}$ & -4.799464 & 4.831004 & -0.373671 & & & & \\
\hline
\end{tabular}




\section{DR4H0}

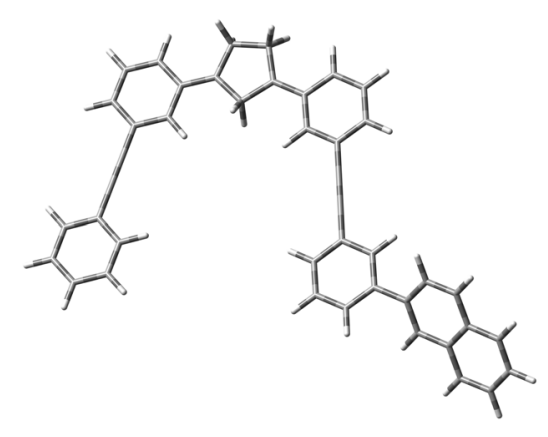

\#p opt freq uwb97xd/6-31g(d)

Charge $=0$, Multiplicity $=3$, Point group $=C_{1}$

Number of Imaginary Frequencies $=0$

Sum of electronic and zero-point Energies $=-1655.341020$ Hartree

Sum of electronic and thermal Energies $=-1655.306078$ Hartree

Sum of electronic and enthalpy Energies = -1655.305133 Hartree

Sum of electronic and thermal Free Energies $=-1655.418186$ Hartree

\begin{tabular}{|c|c|c|c|c|c|c|c|}
\hline \multicolumn{5}{|c|}{ Cartesian Coordinates } & \multicolumn{3}{|c|}{ Cartesian Coordinates } \\
\hline Atom & $X$ & $Y$ & $Z$ & Atom & $\boldsymbol{X}$ & $Y$ & $Z$ \\
\hline $\mathrm{C}$ & -3.935931 & -2.549523 & 0.066364 & $\mathrm{C}$ & -4.279602 & 6.163363 & -0.344326 \\
\hline $\mathrm{C}$ & -3.283528 & -3.900265 & 0.036795 & $\mathrm{C}$ & -6.594241 & 6.805983 & -0.143110 \\
\hline $\mathrm{C}$ & -4.311746 & -4.997782 & 0.032822 & $\mathrm{H}$ & -8.005187 & 5.190204 & 0.052164 \\
\hline $\mathrm{C}$ & -5.676028 & -4.304344 & 0.236231 & $\mathrm{H}$ & -3.884791 & 4.046158 & -0.306415 \\
\hline $\mathrm{C}$ & -5.408114 & -2.827083 & 0.148411 & $\mathrm{H}$ & -3.233572 & 6.432608 & -0.455860 \\
\hline $\mathrm{H}$ & -4.287250 & -5.548214 & -0.919214 & $\mathrm{H}$ & -4.969203 & 8.202924 & -0.351315 \\
\hline $\mathrm{H}$ & -4.115560 & -5.742024 & 0.815603 & $\mathrm{C}$ & 2.916313 & -0.016529 & -0.062825 \\
\hline $\mathrm{H}$ & -6.107513 & -4.562314 & 1.214624 & $\mathrm{C}$ & 4.649958 & 2.169242 & -0.044438 \\
\hline $\mathrm{H}$ & -6.410774 & -4.629849 & -0.511664 & $\mathrm{C}$ & 4.300592 & -0.207001 & -0.152519 \\
\hline $\mathrm{C}$ & -6.410612 & -1.823755 & 0.177617 & $\mathrm{C}$ & 2.406970 & 1.285279 & 0.038219 \\
\hline $\mathrm{C}$ & -8.407487 & 0.180292 & 0.237640 & $\mathrm{C}$ & 3.276453 & 2.367805 & 0.047249 \\
\hline $\mathrm{C}$ & -6.074112 & -0.451209 & 0.098152 & $\mathrm{C}$ & 5.179272 & 0.877473 & -0.142655 \\
\hline $\mathrm{C}$ & -7.785855 & -2.153273 & 0.290459 & $\mathrm{H}$ & 4.688528 & -1.219586 & -0.207539 \\
\hline $\mathrm{C}$ & -8.756379 & -1.165573 & 0.319023 & $\mathrm{H}$ & 1.334436 & 1.434230 & 0.107247 \\
\hline $\mathrm{C}$ & -7.053169 & 0.540756 & 0.126278 & $\mathrm{H}$ & 2.880789 & 3.376565 & 0.116767 \\
\hline $\mathrm{H}$ & -5.034738 & -0.151906 & 0.012262 & $\mathrm{H}$ & 5.320758 & 3.023286 & -0.063344 \\
\hline $\mathrm{H}$ & -8.082330 & -3.195370 & 0.358415 & $\mathrm{C}$ & 6.645403 & 0.660457 & -0.231542 \\
\hline $\mathrm{H}$ & -9.802624 & -1.443283 & 0.406656 & $\mathrm{C}$ & 7.518371 & 1.388517 & 0.544838 \\
\hline $\mathrm{H}$ & -9.166926 & 0.954467 & 0.259648 & $\mathrm{C}$ & 7.172304 & -0.312685 & -1.125013 \\
\hline $\mathrm{C}$ & -1.881592 & -4.110132 & -0.018398 & $\mathrm{C}$ & 8.920099 & 1.190673 & 0.471217 \\
\hline $\mathrm{C}$ & 0.915646 & -4.524621 & -0.129842 & $\mathrm{H}$ & 7.133414 & 2.118598 & 1.253019 \\
\hline $\mathrm{C}$ & -0.978594 & -3.020193 & -0.019124 & $\mathrm{C}$ & 8.520695 & -0.526546 & -1.218802 \\
\hline $\mathrm{C}$ & -1.329032 & -5.415438 & -0.078024 & $\mathrm{H}$ & 6.489178 & -0.873890 & -1.756157 \\
\hline $\mathrm{C}$ & 0.041375 & -5.608742 & -0.132109 & $\mathrm{C}$ & 9.827423 & 1.933394 & 1.272028 \\
\hline $\mathrm{C}$ & 0.400243 & -3.217897 & -0.073073 & $\mathrm{C}$ & 9.434699 & 0.215243 & -0.426074 \\
\hline $\mathrm{H}$ & -1.355582 & -2.003682 & 0.023925 & $\mathrm{H}$ & 8.909268 & -1.266686 & -1.913656 \\
\hline $\mathrm{H}$ & -1.990359 & -6.276297 & -0.083993 & $\mathrm{C}$ & 11.178262 & 1.720295 & 1.180837 \\
\hline
\end{tabular}




\begin{tabular}{cccccccc}
\hline & \multicolumn{3}{c}{ Cartesian Coordinates } & \multicolumn{3}{c}{ Cartesian Coordinates } \\
Atom & $\boldsymbol{X}$ & $\boldsymbol{Y}$ & $\boldsymbol{Z}$ & Atom & $\boldsymbol{X}$ & $\boldsymbol{Y}$ & $\boldsymbol{Z}$ \\
\hline $\mathrm{H}$ & 0.439856 & -6.617991 & -0.177465 & $\mathrm{H}$ & 9.431580 & 2.676179 & 1.959981 \\
$\mathrm{H}$ & 1.989059 & -4.675009 & -0.171393 & $\mathrm{C}$ & 10.837792 & 0.017556 & -0.499104 \\
$\mathrm{C}$ & -6.678924 & 1.919284 & 0.041633 & $\mathrm{C}$ & 11.689867 & 0.751798 & 0.284571 \\
$\mathrm{C}$ & -6.362124 & 3.085449 & -0.031070 & $\mathrm{H}$ & 11.863006 & 2.294930 & 1.797367 \\
$\mathrm{C}$ & 1.285383 & -2.093579 & -0.070266 & $\mathrm{H}$ & 11.226541 & -0.728017 & -1.188114 \\
$\mathrm{C}$ & 2.033444 & -1.141891 & -0.066995 & $\mathrm{H}$ & 12.762150 & 0.592502 & 0.221708 \\
$\mathrm{C}$ & -5.987177 & 4.463088 & -0.117112 & $\mathrm{H}$ & -3.587488 & -1.943391 & 0.921956 \\
$\mathrm{C}$ & -5.253880 & 7.157140 & -0.285767 & $\mathrm{H}$ & -3.679074 & -1.953511 & -0.827839 \\
$\mathrm{C}$ & -6.962287 & 5.469122 & -0.059051 & $\mathrm{H}$ & -7.357097 & 7.577348 & -0.097006 \\
$\mathrm{C}$ & -4.639900 & 4.824357 & -0.260871 & & & & \\
\hline
\end{tabular}




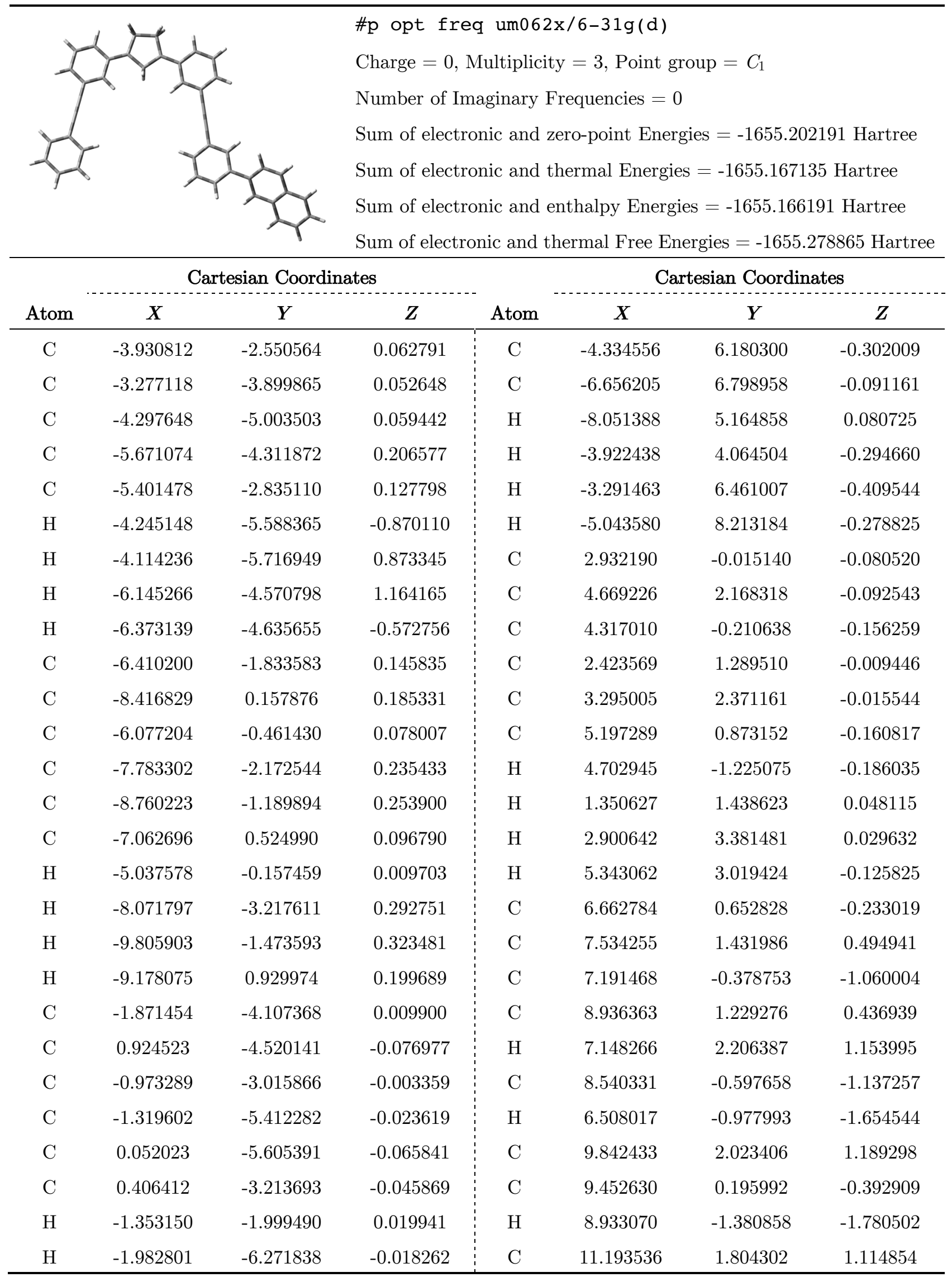




\begin{tabular}{cccccccc}
\hline & \multicolumn{3}{c}{ Cartesian Coordinates } & \multicolumn{3}{c}{ Cartesian Coordinates } \\
Atom & $\boldsymbol{X}$ & $\boldsymbol{Y}$ & $\boldsymbol{Z}$ & Atom & $\boldsymbol{X}$ & $\boldsymbol{Y}$ & $\boldsymbol{Z}$ \\
\hline $\mathrm{H}$ & 0.451632 & -6.614562 & -0.090957 & $\mathrm{H}$ & 9.443321 & 2.809467 & 1.825066 \\
$\mathrm{H}$ & 1.998453 & -4.666637 & -0.109520 & $\mathrm{C}$ & 10.855955 & -0.006762 & -0.450566 \\
$\mathrm{C}$ & -6.696055 & 1.908131 & 0.026745 & $\mathrm{C}$ & 11.707046 & 0.777606 & 0.284575 \\
$\mathrm{C}$ & -6.389491 & 3.078682 & -0.031550 & $\mathrm{H}$ & 11.877366 & 2.417760 & 1.693152 \\
$\mathrm{C}$ & 1.293045 & -2.088474 & -0.058020 & $\mathrm{H}$ & 11.243194 & -0.796944 & -1.088614 \\
$\mathrm{C}$ & 2.045471 & -1.139210 & -0.068374 & $\mathrm{H}$ & 12.779074 & 0.614665 & 0.234573 \\
$\mathrm{C}$ & -6.026875 & 4.462018 & -0.099337 & $\mathrm{H}$ & -3.593838 & -1.935826 & 0.917427 \\
$\mathrm{C}$ & -5.318502 & 7.164297 & -0.228718 & $\mathrm{H}$ & -3.664591 & -1.959246 & -0.832389 \\
$\mathrm{C}$ & -7.012095 & 5.457610 & -0.026620 & $\mathrm{H}$ & -7.425578 & 7.562589 & -0.033668 \\
$\mathrm{C}$ & -4.682476 & 4.836837 & -0.238058 & & & & \\
\hline
\end{tabular}




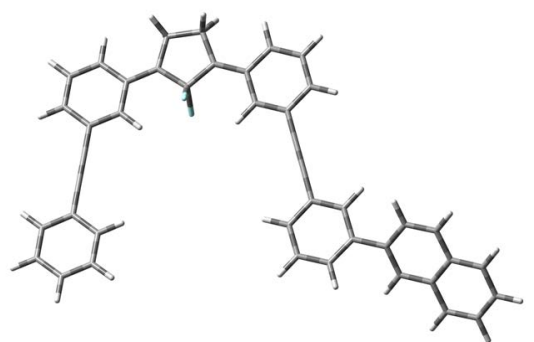

\#p opt freq ub3lyp/6-31g(d)

Charge $=0$, Multiplicity $=3$, Point group $=C_{1}$

Number of Imaginary Frequencies $=0$

Sum of electronic and zero-point Energies $=-1854.410400$ Hartree

Sum of electronic and thermal Energies $=-1854.373486$ Hartree

Sum of electronic and enthalpy Energies $=-1854.372541$ Hartree

Sum of electronic and thermal Free Energies $=-1854.489359$ Hartree

\begin{tabular}{|c|c|c|c|c|c|c|c|}
\hline \multirow[b]{2}{*}{ Atom } & \multicolumn{3}{|c|}{ Cartesian Coordinates } & \multirow[b]{2}{*}{ Atom } & \multicolumn{3}{|c|}{ Cartesian Coordinates } \\
\hline & $X$ & $Y$ & $Z$ & & $X$ & $Y$ & $Z$ \\
\hline $\mathrm{C}$ & -3.786550 & -2.451865 & 0.060778 & $\mathrm{C}$ & -4.178894 & 6.321590 & -0.500890 \\
\hline $\mathrm{C}$ & -3.119971 & -3.796067 & -0.003877 & $\mathrm{C}$ & -6.491866 & 6.957580 & -0.203280 \\
\hline $\mathrm{C}$ & -4.167826 & -4.874622 & -0.056926 & $\mathrm{H}$ & -7.885700 & 5.336468 & 0.079500 \\
\hline $\mathrm{C}$ & -5.509446 & -4.184018 & 0.306147 & $\mathrm{H}$ & -3.769883 & 4.204410 & -0.450142 \\
\hline $\mathrm{C}$ & -5.262321 & -2.705568 & 0.174751 & $\mathrm{H}$ & -3.139638 & 6.594192 & -0.663814 \\
\hline $\mathrm{H}$ & -4.221399 & -5.312466 & -1.066348 & $\mathrm{H}$ & -4.881727 & 8.361796 & -0.506067 \\
\hline $\mathrm{H}$ & -3.945100 & -5.707605 & 0.621323 & $\mathrm{C}$ & 3.138609 & 0.050612 & 0.006804 \\
\hline $\mathrm{H}$ & -5.805537 & -4.436495 & 1.336717 & $\mathrm{C}$ & 4.908605 & 2.219693 & 0.112793 \\
\hline $\mathrm{H}$ & -6.328978 & -4.529652 & -0.336020 & $\mathrm{C}$ & 4.526330 & -0.149136 & -0.113149 \\
\hline $\mathrm{C}$ & -6.251007 & -1.686522 & 0.229288 & $\mathrm{C}$ & 2.647876 & 1.359240 & 0.182663 \\
\hline $\mathrm{C}$ & -8.248642 & 0.320920 & 0.350434 & $\mathrm{C}$ & 3.535392 & 2.429859 & 0.234568 \\
\hline $\mathrm{C}$ & -5.913916 & -0.310113 & 0.114236 & $\mathrm{C}$ & 5.425593 & 0.923807 & -0.060594 \\
\hline $\mathrm{C}$ & -7.625006 & -2.016286 & 0.406600 & $\mathrm{H}$ & 4.898574 & -1.162659 & -0.223342 \\
\hline $\mathrm{C}$ & -8.595634 & -1.025864 & 0.465068 & $\mathrm{H}$ & 1.578293 & 1.518750 & 0.274671 \\
\hline $\mathrm{C}$ & -6.894488 & 0.686561 & 0.173133 & $\mathrm{H}$ & 3.154285 & 3.439648 & 0.360758 \\
\hline $\mathrm{H}$ & -4.880941 & -0.020147 & -0.024440 & $\mathrm{H}$ & 5.587358 & 3.067462 & 0.125465 \\
\hline $\mathrm{H}$ & -7.917301 & -3.057126 & 0.501881 & $\mathrm{C}$ & 6.888287 & 0.694414 & -0.183116 \\
\hline $\mathrm{H}$ & -9.637702 & -1.301717 & 0.602569 & $\mathrm{C}$ & 7.793580 & 1.420372 & 0.572642 \\
\hline $\mathrm{H}$ & -9.007499 & 1.095228 & 0.395681 & $\mathrm{C}$ & 7.392385 & -0.286977 & -1.086640 \\
\hline $\mathrm{C}$ & -1.715104 & -3.993304 & -0.083670 & $\mathrm{C}$ & 9.192956 & 1.212924 & 0.468272 \\
\hline $\mathrm{C}$ & 1.081829 & -4.422870 & -0.252963 & $\mathrm{H}$ & 7.433012 & 2.154235 & 1.289324 \\
\hline $\mathrm{C}$ & -0.803042 & -2.903417 & -0.042790 & $\mathrm{C}$ & 8.742113 & -0.511948 & -1.212354 \\
\hline $\mathrm{C}$ & -1.171547 & -5.303310 & -0.211921 & $\mathrm{H}$ & 6.693168 & -0.844296 & -1.703269 \\
\hline $\mathrm{C}$ & 0.199485 & -5.503203 & -0.294491 & $\mathrm{C}$ & 10.127143 & 1.950419 & 1.245909 \\
\hline $\mathrm{C}$ & 0.578831 & -3.107735 & -0.125878 & $\mathrm{C}$ & 9.683783 & 0.223993 & -0.444521 \\
\hline $\mathrm{H}$ & -1.177521 & -1.893427 & 0.057386 & $\mathrm{H}$ & 9.107305 & -1.257066 & -1.915441 \\
\hline $\mathrm{H}$ & -1.839308 & -6.157993 & -0.250135 & $\mathrm{C}$ & 11.479718 & 1.725952 & 1.123215 \\
\hline
\end{tabular}




\begin{tabular}{cccccccc}
\hline & \multicolumn{3}{c}{ Cartesian Coordinates } & \multicolumn{3}{c}{ Cartesian Coordinates } \\
Atom & $\boldsymbol{X}$ & $\boldsymbol{Y}$ & $\boldsymbol{Z}$ & Atom & $\boldsymbol{X}$ & $\boldsymbol{Y}$ & $\boldsymbol{Z}$ \\
\hline $\mathrm{H}$ & 0.590774 & -6.512162 & -0.393374 & $\mathrm{H}$ & 9.753589 & 2.698652 & 1.941213 \\
$\mathrm{H}$ & 2.153743 & -4.579055 & -0.316657 & $\mathrm{C}$ & 11.084748 & 0.017178 & -0.548433 \\
$\mathrm{C}$ & -6.532719 & 2.060743 & 0.053687 & $\mathrm{C}$ & 11.963981 & 0.750448 & 0.216731 \\
$\mathrm{C}$ & -6.231291 & 3.234435 & -0.048826 & $\mathrm{H}$ & 12.183022 & 2.297694 & 1.722676 \\
$\mathrm{C}$ & 1.474531 & -1.998928 & -0.080768 & $\mathrm{H}$ & 11.452735 & -0.733201 & -1.244441 \\
$\mathrm{C}$ & 2.243879 & -1.058055 & -0.041090 & $\mathrm{H}$ & 13.034026 & 0.582778 & 0.129124 \\
$\mathrm{C}$ & -5.870524 & 4.607751 & -0.170635 & $\mathrm{~F}$ & -3.319460 & -1.689948 & 1.128042 \\
$\mathrm{C}$ & -5.157460 & 7.314977 & -0.412421 & $\mathrm{~F}$ & -3.475772 & -1.678418 & -1.054073 \\
$\mathrm{C}$ & -6.849722 & 5.617674 & -0.082992 & $\mathrm{H}$ & -7.256886 & 7.726253 & -0.133733 \\
$\mathrm{C}$ & -4.527288 & 4.979132 & -0.381624 & & & & \\
\hline
\end{tabular}




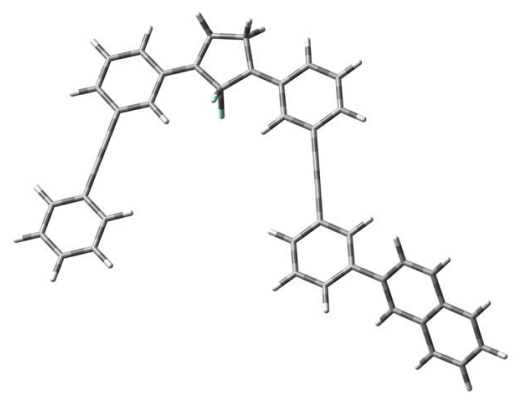

\#p opt freq uwb97xd/6-31g(d)

Charge $=0$, Multiplicity $=3$, Point group $=C_{1}$

Number of Imaginary Frequencies $=0$

Sum of electronic and zero-point Energies $=-1853.779343$ Hartree

Sum of electronic and thermal Energies $=-1853.742851$ Hartree

Sum of electronic and enthalpy Energies = -1853.741906 Hartree

Sum of electronic and thermal Free Energies $=-1853.858039$ Hartree

\begin{tabular}{|c|c|c|c|c|c|c|c|}
\hline \multicolumn{5}{|c|}{ Cartesian Coordinates } & \multicolumn{3}{|c|}{ Cartesian Coordinates } \\
\hline Atom & $\boldsymbol{X}$ & $Y$ & $Z$ & Atom & $X$ & $Y$ & $Z$ \\
\hline $\mathrm{C}$ & -3.775558 & -2.435092 & 0.062044 & $\mathrm{C}$ & -4.107228 & 6.301135 & -0.450892 \\
\hline $\mathrm{C}$ & -3.118752 & -3.782580 & 0.018883 & $\mathrm{C}$ & -6.409912 & 6.957971 & -0.170429 \\
\hline $\mathrm{C}$ & -4.162828 & -4.859431 & -0.014259 & $\mathrm{H}$ & -7.821068 & 5.351389 & 0.088721 \\
\hline $\mathrm{C}$ & -5.511478 & -4.159621 & 0.275260 & $\mathrm{H}$ & -3.722307 & 4.181770 & -0.410835 \\
\hline $\mathrm{C}$ & -5.250769 & -2.686676 & 0.158615 & $\mathrm{H}$ & -3.064633 & 6.563881 & -0.602607 \\
\hline $\mathrm{H}$ & -4.179726 & -5.345428 & -1.000653 & $\mathrm{H}$ & -4.786311 & 8.344172 & -0.448612 \\
\hline $\mathrm{H}$ & -3.956429 & -5.652812 & 0.713897 & $\mathrm{C}$ & 3.126429 & 0.044918 & -0.039275 \\
\hline $\mathrm{H}$ & -5.869188 & -4.405749 & 1.285652 & $\mathrm{C}$ & 4.877555 & 2.214786 & 0.023775 \\
\hline $\mathrm{H}$ & -6.294553 & -4.493195 & -0.415944 & $\mathrm{C}$ & 4.508307 & -0.154885 & -0.142656 \\
\hline $\mathrm{C}$ & -6.236271 & -1.666845 & 0.209544 & $\mathrm{C}$ & 2.627718 & 1.347466 & 0.098246 \\
\hline $\mathrm{C}$ & -8.223732 & 0.334865 & 0.321960 & $\mathrm{C}$ & 3.506309 & 2.422182 & 0.129271 \\
\hline $\mathrm{C}$ & -5.895128 & -0.296368 & 0.106552 & $\mathrm{C}$ & 5.395922 & 0.921848 & -0.110670 \\
\hline $\mathrm{C}$ & -7.605632 & -1.996436 & 0.370959 & $\mathrm{H}$ & 4.887615 & -1.168828 & -0.225926 \\
\hline $\mathrm{C}$ & -8.574427 & -1.008203 & 0.425351 & $\mathrm{H}$ & 1.556853 & 1.502653 & 0.177867 \\
\hline $\mathrm{C}$ & -6.873499 & 0.693881 & 0.161615 & $\mathrm{H}$ & 3.119417 & 3.431967 & 0.227023 \\
\hline $\mathrm{H}$ & -4.860835 & -0.003440 & -0.018924 & $\mathrm{H}$ & 5.555195 & 3.063645 & 0.022434 \\
\hline $\mathrm{H}$ & -7.899933 & -3.037543 & 0.457933 & $\mathrm{C}$ & 6.859659 & 0.695351 & -0.213927 \\
\hline $\mathrm{H}$ & -9.617261 & -1.283239 & 0.550894 & $\mathrm{C}$ & 7.743124 & 1.396403 & 0.575334 \\
\hline $\mathrm{H}$ & -8.980644 & 1.110803 & 0.364076 & $\mathrm{C}$ & 7.373384 & -0.259212 & -1.134703 \\
\hline $\mathrm{C}$ & -1.716737 & -3.988173 & -0.057239 & $\mathrm{C}$ & 9.142709 & 1.188972 & 0.488489 \\
\hline $\mathrm{C}$ & 1.066427 & -4.433824 & -0.216826 & $\mathrm{H}$ & 7.368288 & 2.111555 & 1.303875 \\
\hline $\mathrm{C}$ & -0.804217 & -2.905625 & -0.034806 & $\mathrm{C}$ & 8.719380 & -0.481813 & -1.241715 \\
\hline $\mathrm{C}$ & -1.185176 & -5.298161 & -0.162898 & $\mathrm{H}$ & 6.682024 & -0.798521 & -1.775906 \\
\hline $\mathrm{C}$ & 0.181617 & -5.508095 & -0.240666 & $\mathrm{C}$ & 10.060741 & 1.903869 & 1.302320 \\
\hline $\mathrm{C}$ & 0.569632 & -3.122266 & -0.113647 & $\mathrm{C}$ & 9.644058 & 0.232175 & -0.435942 \\
\hline $\mathrm{H}$ & -1.170344 & -1.890407 & 0.046909 & $\mathrm{H}$ & 9.097806 & -1.207354 & -1.957267 \\
\hline $\mathrm{H}$ & -1.858205 & -6.149370 & -0.187570 & $\mathrm{C}$ & 11.409214 & 1.681999 & 1.197948 \\
\hline
\end{tabular}




\begin{tabular}{cccccccc}
\hline & \multicolumn{3}{c}{ Cartesian Coordinates } & \multicolumn{3}{c}{ Cartesian Coordinates } \\
Atom & $\boldsymbol{X}$ & $\boldsymbol{Y}$ & $\boldsymbol{Z}$ & Atom & $\boldsymbol{X}$ & $\boldsymbol{Y}$ & $\boldsymbol{Z}$ \\
\hline $\mathrm{H}$ & 0.566909 & -6.519941 & -0.322011 & $\mathrm{H}$ & 9.675010 & 2.632444 & 2.010895 \\
$\mathrm{H}$ & 2.137403 & -4.595552 & -0.277006 & $\mathrm{C}$ & 11.045007 & 0.024799 & -0.522054 \\
$\mathrm{C}$ & -6.504013 & 2.071723 & 0.053913 & $\mathrm{C}$ & 11.907630 & 0.732079 & 0.274778 \\
$\mathrm{C}$ & -6.191165 & 3.237322 & -0.038281 & $\mathrm{H}$ & 12.102206 & 2.235370 & 1.824639 \\
$\mathrm{C}$ & 1.471337 & -2.011932 & -0.088437 & $\mathrm{H}$ & 11.423619 & -0.706435 & -1.231771 \\
$\mathrm{C}$ & 2.233095 & -1.071752 & -0.066346 & $\mathrm{H}$ & 12.978169 & 0.565569 & 0.201669 \\
$\mathrm{C}$ & -5.813977 & 4.612577 & -0.148398 & $\mathrm{~F}$ & -3.324303 & -1.676887 & 1.123172 \\
$\mathrm{C}$ & -5.073777 & 7.300463 & -0.364585 & $\mathrm{~F}$ & -3.453338 & -1.683233 & -1.049475 \\
$\mathrm{C}$ & -6.781471 & 5.623791 & -0.062623 & $\mathrm{H}$ & -7.166486 & 7.733887 & -0.102554 \\
$\mathrm{C}$ & -4.470815 & 4.964710 & -0.343945 & & & & \\
\hline
\end{tabular}




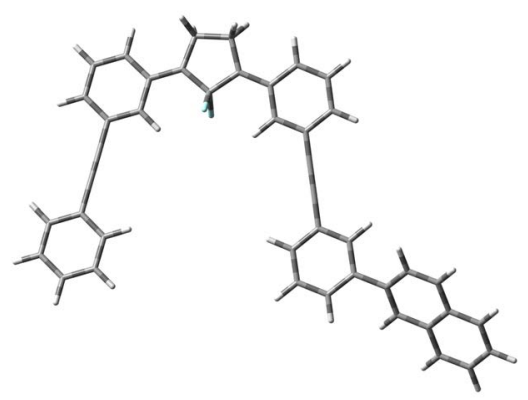

\#p opt freq um062x/6-31g(d)

Charge $=0$, Multiplicity $=3$, Point group $=C_{1}$

Number of Imaginary Frequencies $=0$

Sum of electronic and zero-point Energies $=-1853.637159$ Hartree

Sum of electronic and thermal Energies $=-1853.600558$ Hartree

Sum of electronic and enthalpy Energies = -1853.599614 Hartree

Sum of electronic and thermal Free Energies $=-1853.715597$ Hartree

\begin{tabular}{|c|c|c|c|c|c|c|c|}
\hline \multicolumn{5}{|c|}{ Cartesian Coordinates } & \multicolumn{3}{|c|}{ Cartesian Coordinates } \\
\hline Atom & $\boldsymbol{X}$ & $Y$ & $Z$ & Atom & $X$ & $Y$ & $Z$ \\
\hline $\mathrm{C}$ & -3.801740 & -2.439322 & 0.062071 & $\mathrm{C}$ & -3.985271 & 6.310396 & -0.463304 \\
\hline $\mathrm{C}$ & -3.160652 & -3.793184 & 0.027634 & $\mathrm{C}$ & -6.275715 & 6.990812 & -0.132555 \\
\hline $\mathrm{C}$ & -4.210777 & -4.862895 & -0.009074 & $\mathrm{H}$ & -7.699264 & 5.394973 & 0.137526 \\
\hline $\mathrm{C}$ & -5.557370 & -4.148654 & 0.265828 & $\mathrm{H}$ & -3.626066 & 4.184572 & -0.450909 \\
\hline $\mathrm{C}$ & -5.278029 & -2.680169 & 0.147653 & $\mathrm{H}$ & -2.943219 & 6.563144 & -0.632294 \\
\hline $\mathrm{H}$ & -4.223064 & -5.354026 & -0.992720 & $\mathrm{H}$ & -4.641374 & 8.360768 & -0.428113 \\
\hline $\mathrm{H}$ & -4.014998 & -5.653408 & 0.724671 & $\mathrm{C}$ & 3.094540 & 0.036955 & -0.035082 \\
\hline $\mathrm{H}$ & -5.927826 & -4.388261 & 1.272938 & $\mathrm{C}$ & 4.860025 & 2.195550 & 0.026032 \\
\hline $\mathrm{H}$ & -6.337644 & -4.473786 & -0.432083 & $\mathrm{C}$ & 4.475370 & -0.173351 & -0.137335 \\
\hline $\mathrm{C}$ & -6.245426 & -1.639785 & 0.193096 & $\mathrm{C}$ & 2.603663 & 1.343312 & 0.099699 \\
\hline $\mathrm{C}$ & -8.184635 & 0.402662 & 0.291526 & $\mathrm{C}$ & 3.489671 & 2.412654 & 0.129573 \\
\hline $\mathrm{C}$ & -5.867443 & -0.280304 & 0.097390 & $\mathrm{C}$ & 5.370031 & 0.898084 & -0.105772 \\
\hline $\mathrm{C}$ & -7.620470 & -1.941947 & 0.340499 & $\mathrm{H}$ & 4.847217 & -1.190401 & -0.216501 \\
\hline $\mathrm{C}$ & -8.568001 & -0.931780 & 0.388135 & $\mathrm{H}$ & 1.533573 & 1.502965 & 0.177823 \\
\hline $\mathrm{C}$ & -6.824863 & 0.731419 & 0.145480 & $\mathrm{H}$ & 3.109635 & 3.424985 & 0.224389 \\
\hline $\mathrm{H}$ & -4.823212 & -0.015210 & -0.015796 & $\mathrm{H}$ & 5.544743 & 3.038598 & 0.021471 \\
\hline $\mathrm{H}$ & -7.933916 & -2.978238 & 0.421117 & $\mathrm{C}$ & 6.831421 & 0.661995 & -0.205666 \\
\hline $\mathrm{H}$ & -9.617829 & -1.182739 & 0.502616 & $\mathrm{C}$ & 7.721904 & 1.396336 & 0.545504 \\
\hline $\mathrm{H}$ & -8.921605 & 1.197502 & 0.327661 & $\mathrm{C}$ & 7.336301 & -0.338877 & -1.083714 \\
\hline $\mathrm{C}$ & -1.756039 & -3.998532 & -0.044101 & $\mathrm{C}$ & 9.120292 & 1.177472 & 0.461907 \\
\hline $\mathrm{C}$ & 1.024613 & -4.433279 & -0.192687 & $\mathrm{H}$ & 7.354284 & 2.145854 & 1.242711 \\
\hline $\mathrm{C}$ & -0.853297 & -2.910033 & -0.027014 & $\mathrm{C}$ & 8.680999 & -0.572441 & -1.186531 \\
\hline $\mathrm{C}$ & -1.223186 & -5.306546 & -0.139106 & $\mathrm{H}$ & 6.637788 & -0.901771 & -1.696007 \\
\hline $\mathrm{C}$ & 0.145442 & -5.512039 & -0.211826 & $\mathrm{C}$ & 10.045833 & 1.925176 & 1.237777 \\
\hline $\mathrm{C}$ & 0.522091 & -3.122897 & -0.100103 & $\mathrm{C}$ & 9.612685 & 0.175007 & -0.418736 \\
\hline $\mathrm{H}$ & -1.228477 & -1.896399 & 0.045150 & $\mathrm{H}$ & 9.055410 & -1.331623 & -1.868346 \\
\hline $\mathrm{H}$ & -1.897045 & -6.157536 & -0.158912 & $\mathrm{C}$ & 11.392796 & 1.691125 & 1.137995 \\
\hline
\end{tabular}




\begin{tabular}{cccccccc}
\hline & \multicolumn{3}{c}{ Cartesian Coordinates } & \multicolumn{3}{c}{ Cartesian Coordinates } \\
Atom & $\boldsymbol{X}$ & $\boldsymbol{Y}$ & $\boldsymbol{Z}$ & Atom & $\boldsymbol{X}$ & $\boldsymbol{Y}$ & $\boldsymbol{Z}$ \\
\hline $\mathrm{H}$ & 0.535399 & -6.522368 & -0.284991 & $\mathrm{H}$ & 9.664963 & 2.687801 & 1.912095 \\
$\mathrm{H}$ & 2.096638 & -4.587755 & -0.248331 & $\mathrm{C}$ & 11.012272 & -0.044113 & -0.501698 \\
$\mathrm{C}$ & -6.427497 & 2.103801 & 0.044782 & $\mathrm{C}$ & 11.882483 & 0.695217 & 0.257362 \\
$\mathrm{C}$ & -6.098351 & 3.266203 & -0.041495 & $\mathrm{H}$ & 12.091644 & 2.269067 & 1.734634 \\
$\mathrm{C}$ & 1.424303 & -2.010688 & -0.079107 & $\mathrm{H}$ & 11.381176 & -0.810546 & -1.178404 \\
$\mathrm{C}$ & 2.192607 & -1.074689 & -0.059580 & $\mathrm{H}$ & 12.951466 & 0.519932 & 0.187740 \\
$\mathrm{C}$ & -5.705661 & 4.639039 & -0.145030 & $\mathrm{~F}$ & -3.351391 & -1.683266 & 1.119881 \\
$\mathrm{C}$ & -4.939068 & 7.319905 & -0.348821 & $\mathrm{~F}$ & -3.464899 & -1.693051 & -1.043849 \\
$\mathrm{C}$ & -6.660581 & 5.659815 & -0.030852 & $\mathrm{H}$ & -7.021455 & 7.774519 & -0.042832 \\
$\mathrm{C}$ & -4.361991 & 4.976967 & -0.362519 & & & & \\
\hline
\end{tabular}




\section{DR4H1}

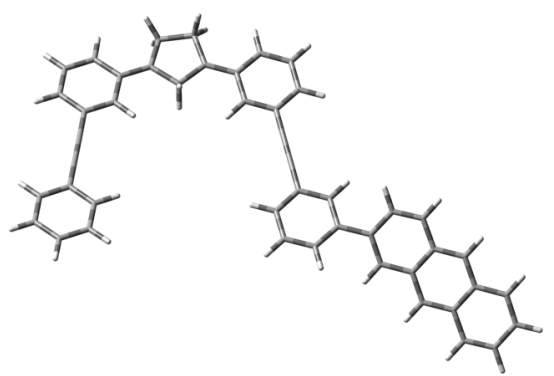

\#p opt freq ub3lyp/6-31g(d)

Charge $=0$, Multiplicity $=3$, Point group $=C_{1}$

Number of Imaginary Frequencies $=0$

Sum of electronic and zero-point Energies $=-1809.513067$ Hartree

Sum of electronic and thermal Energies $=-1809.475059$ Hartree

Sum of electronic and enthalpy Energies $=-1809.474114$ Hartree

Sum of electronic and thermal Free Energies $=-1809.59366$ Hartree

\begin{tabular}{|c|c|c|c|c|c|c|c|}
\hline \multirow[b]{2}{*}{ Atom } & \multicolumn{3}{|c|}{ Cartesian Coordinates } & \multirow[b]{2}{*}{ Atom } & \multicolumn{3}{|c|}{ Cartesian Coordinates } \\
\hline & $\boldsymbol{X}$ & $\boldsymbol{Y}$ & $Z$ & & $X$ & $Y$ & $Z$ \\
\hline $\mathrm{C}$ & -5.145725 & -2.485761 & 0.131552 & $\mathrm{H}$ & -4.963509 & 4.133544 & -0.490656 \\
\hline $\mathrm{C}$ & -4.537374 & -3.861870 & 0.057995 & $\mathrm{H}$ & -4.275177 & 6.500473 & -0.765079 \\
\hline $\mathrm{C}$ & -5.616120 & -4.915602 & 0.057554 & $\mathrm{H}$ & -5.966263 & 8.317052 & -0.607540 \\
\hline $\mathrm{C}$ & -6.918141 & -4.182647 & 0.462981 & $\mathrm{C}$ & 1.830148 & -0.224309 & -0.148645 \\
\hline $\mathrm{C}$ & -6.624989 & -2.711941 & 0.304375 & $\mathrm{C}$ & 3.669015 & 1.890569 & -0.131016 \\
\hline $\mathrm{H}$ & -5.721612 & -5.362017 & -0.944905 & $\mathrm{C}$ & 3.208595 & -0.470194 & -0.289440 \\
\hline $\mathrm{H}$ & -5.389585 & -5.750408 & 0.733366 & $\mathrm{C}$ & 1.384649 & 1.103415 & 0.002978 \\
\hline $\mathrm{H}$ & -7.181563 & -4.415030 & 1.507943 & $\mathrm{C}$ & 2.305765 & 2.146658 & 0.010841 \\
\hline $\mathrm{H}$ & -7.775372 & -4.508615 & -0.140190 & $\mathrm{C}$ & 4.142053 & 0.574718 & -0.279766 \\
\hline $\mathrm{C}$ & -7.597084 & -1.676996 & 0.362733 & $\mathrm{H}$ & 3.546087 & -1.497587 & -0.381177 \\
\hline $\mathrm{C}$ & -9.539765 & 0.394200 & 0.482057 & $\mathrm{H}$ & 0.322530 & 1.299551 & 0.110127 \\
\hline $\mathrm{C}$ & -7.231019 & -0.312143 & 0.214231 & $\mathrm{H}$ & 1.958645 & 3.170889 & 0.117501 \\
\hline $\mathrm{C}$ & -8.977181 & -1.960334 & 0.576132 & $\mathrm{H}$ & 4.373830 & 2.716502 & -0.153915 \\
\hline $\mathrm{C}$ & -9.917968 & -0.941084 & 0.632758 & $\mathrm{H}$ & -4.722679 & -1.891272 & 0.963108 \\
\hline $\mathrm{C}$ & -8.180105 & 0.716074 & 0.270024 & $\mathrm{H}$ & -4.921849 & -1.890338 & -0.773526 \\
\hline $\mathrm{H}$ & -6.192057 & -0.047014 & 0.051090 & $\mathrm{C}$ & 5.593851 & 0.295756 & -0.420059 \\
\hline $\mathrm{H}$ & -9.297822 & -2.989804 & 0.700531 & $\mathrm{C}$ & 6.532634 & 1.012668 & 0.290008 \\
\hline $\mathrm{H}$ & -10.964129 & -1.185514 & 0.797744 & $\mathrm{C}$ & 6.048160 & -0.739776 & -1.304923 \\
\hline $\mathrm{H}$ & -10.276032 & 1.189949 & 0.526108 & $\mathrm{C}$ & 7.931205 & 0.756209 & 0.168163 \\
\hline $\mathrm{C}$ & -3.144560 & -4.122540 & -0.049599 & $\mathrm{H}$ & 6.212933 & 1.781599 & 0.988924 \\
\hline $\mathrm{C}$ & -0.358578 & -4.642254 & -0.267079 & $\mathrm{C}$ & 7.379218 & -1.016509 & -1.451918 \\
\hline $\mathrm{C}$ & -2.194624 & -3.065902 & -0.053006 & $\mathrm{H}$ & 5.314904 & -1.290927 & -1.886371 \\
\hline $\mathrm{C}$ & -2.640031 & -5.450406 & -0.162427 & $\mathrm{C}$ & 8.888065 & 1.479104 & 0.892947 \\
\hline $\mathrm{C}$ & -1.277312 & -5.693290 & -0.268062 & $\mathrm{C}$ & 8.372117 & -0.287477 & -0.726793 \\
\hline $\mathrm{C}$ & -0.819903 & -3.310905 & -0.158401 & $\mathrm{H}$ & 7.703723 & -1.796460 & -2.136767 \\
\hline $\mathrm{H}$ & -2.530843 & -2.038038 & 0.029422 & $\mathrm{C}$ & 10.258732 & 1.220088 & 0.767738 \\
\hline $\mathrm{H}$ & -3.332927 & -6.285826 & -0.169741 & $\mathrm{H}$ & 8.557407 & 2.263886 & 1.570542 \\
\hline
\end{tabular}




\begin{tabular}{cccccccc}
\hline & \multicolumn{3}{c}{ Cartesian Coordinates } & \multicolumn{4}{c}{ Cartesian Coordinates } \\
Atom & $\boldsymbol{X}$ & $\boldsymbol{Y}$ & $\boldsymbol{Z}$ & Atom & $\boldsymbol{X}$ & $\boldsymbol{Y}$ & $\boldsymbol{Z}$ \\
\hline $\mathrm{H}$ & -0.919962 & -6.716214 & -0.353610 & $\mathrm{C}$ & 9.741191 & -0.546759 & -0.853977 \\
$\mathrm{H}$ & 0.706341 & -4.833253 & -0.348918 & $\mathrm{C}$ & 11.244444 & 1.952311 & 1.500633 \\
$\mathrm{C}$ & -7.778719 & 2.075809 & 0.113996 & $\mathrm{C}$ & 10.698688 & 0.178091 & -0.132061 \\
$\mathrm{C}$ & -7.442107 & 3.237042 & -0.019329 & $\mathrm{H}$ & 10.071195 & -1.332536 & -1.530783 \\
$\mathrm{C}$ & 0.106771 & -2.226551 & -0.155651 & $\mathrm{C}$ & 12.578674 & 1.676583 & 1.356885 \\
$\mathrm{C}$ & 0.901273 & -1.305632 & -0.152751 & $\mathrm{H}$ & 10.911332 & 2.735742 & 2.177363 \\
$\mathrm{C}$ & -7.046782 & 4.597249 & -0.176403 & $\mathrm{C}$ & 12.100218 & -0.076535 & -0.251794 \\
$\mathrm{C}$ & -6.267467 & 7.280032 & -0.487242 & $\mathrm{C}$ & 13.012492 & 0.648598 & 0.468814 \\
$\mathrm{C}$ & -7.996602 & 5.635074 & -0.089291 & $\mathrm{H}$ & 13.316473 & 2.241261 & 1.920422 \\
$\mathrm{C}$ & -5.699080 & 4.929132 & -0.422186 & $\mathrm{H}$ & 12.428540 & -0.861141 & -0.929518 \\
$\mathrm{C}$ & -5.317688 & 6.259120 & -0.575557 & $\mathrm{H}$ & 14.074878 & 0.444005 & 0.367658 \\
$\mathrm{C}$ & -7.606087 & 6.962295 & -0.243826 & $\mathrm{H}$ & -8.349103 & 7.752264 & -0.174198 \\
$\mathrm{H}$ & -9.036019 & 5.385024 & 0.099869 & & & & \\
\hline
\end{tabular}




\section{DR4H1}

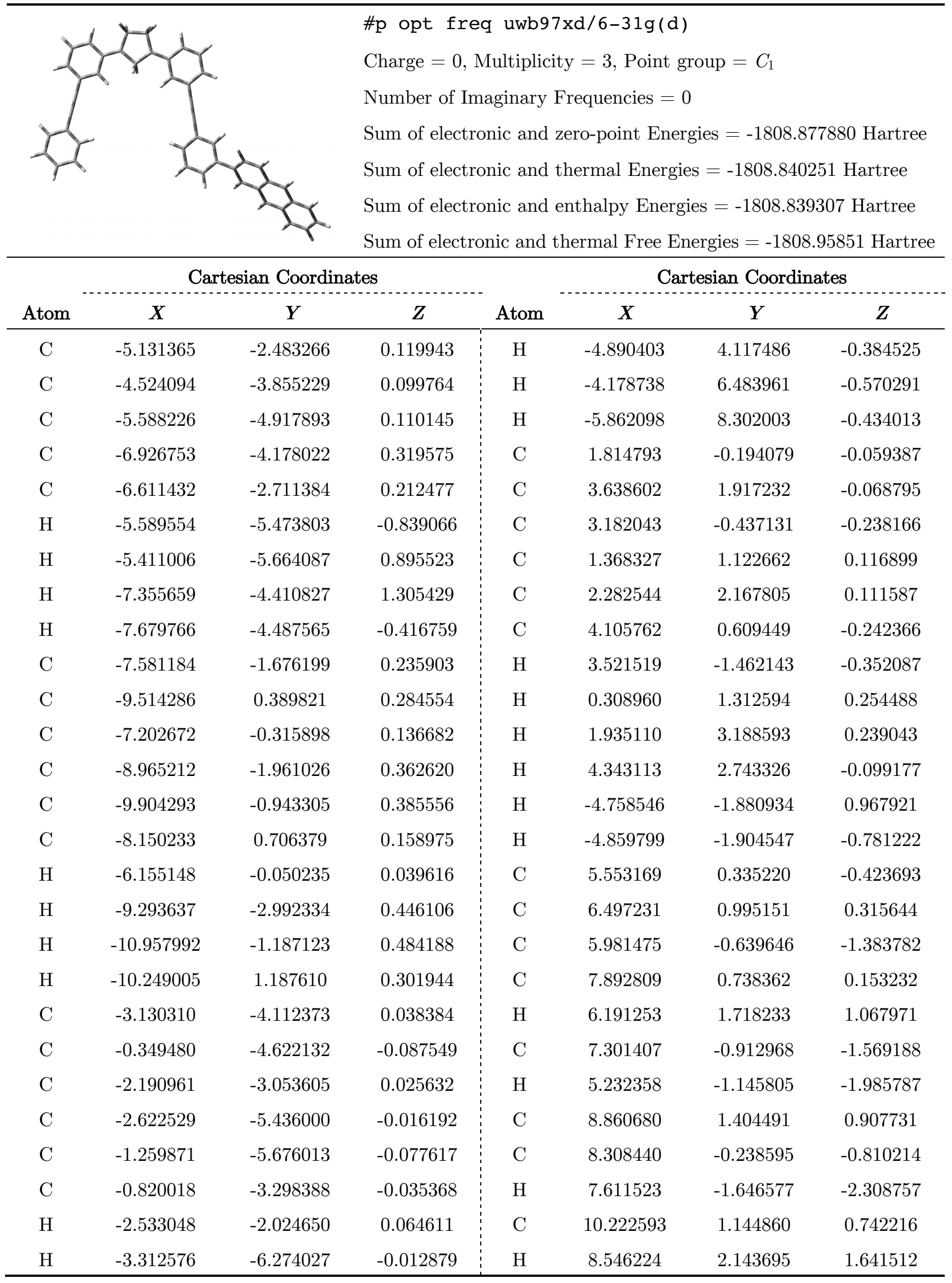




\begin{tabular}{cccccccc}
\hline & \multicolumn{3}{c}{ Cartesian Coordinates } & \multicolumn{4}{c}{ Cartesian Coordinates } \\
Atom & $\boldsymbol{X}$ & $\boldsymbol{Y}$ & $\boldsymbol{Z}$ & Atom & $\boldsymbol{X}$ & $\boldsymbol{Y}$ & $\boldsymbol{Z}$ \\
\hline $\mathrm{H}$ & -0.896112 & -6.698455 & -0.119128 & $\mathrm{C}$ & 9.668922 & -0.498316 & -0.977373 \\
$\mathrm{H}$ & 0.717968 & -4.808964 & -0.134584 & $\mathrm{C}$ & 11.224125 & 1.821229 & 1.507639 \\
$\mathrm{C}$ & -7.734870 & 2.071663 & 0.053855 & $\mathrm{C}$ & 10.637660 & 0.169603 & -0.225058 \\
$\mathrm{C}$ & -7.385737 & 3.227350 & -0.036150 & $\mathrm{H}$ & 9.982576 & -1.238807 & -1.710230 \\
$\mathrm{C}$ & 0.103815 & -2.205649 & -0.044362 & $\mathrm{C}$ & 12.546854 & 1.548407 & 1.325267 \\
$\mathrm{C}$ & 0.886986 & -1.282673 & -0.051022 & $\mathrm{H}$ & 10.906739 & 2.559187 & 2.239871 \\
$\mathrm{C}$ & -6.974899 & 4.593257 & -0.142903 & $\mathrm{C}$ & 12.035590 & -0.086772 & -0.385150 \\
$\mathrm{C}$ & -6.173286 & 7.264932 & -0.352514 & $\mathrm{C}$ & 12.959183 & 0.579294 & 0.363736 \\
$\mathrm{C}$ & -7.920323 & 5.626127 & -0.067125 & $\mathrm{H}$ & 13.297593 & 2.068550 & 1.912583 \\
$\mathrm{C}$ & -5.622599 & 4.916374 & -0.325097 & $\mathrm{H}$ & 12.346540 & -0.826149 & -1.118727 \\
$\mathrm{C}$ & -5.228368 & 6.244379 & -0.428829 & $\mathrm{H}$ & 14.017759 & 0.375842 & 0.232911 \\
$\mathrm{C}$ & -7.518426 & 6.951714 & -0.171594 & $\mathrm{H}$ & -8.258521 & 7.744012 & -0.111513 \\
$\mathrm{H}$ & -8.967107 & 5.376746 & 0.073896 & & & & \\
\hline
\end{tabular}




\section{DR4H1}

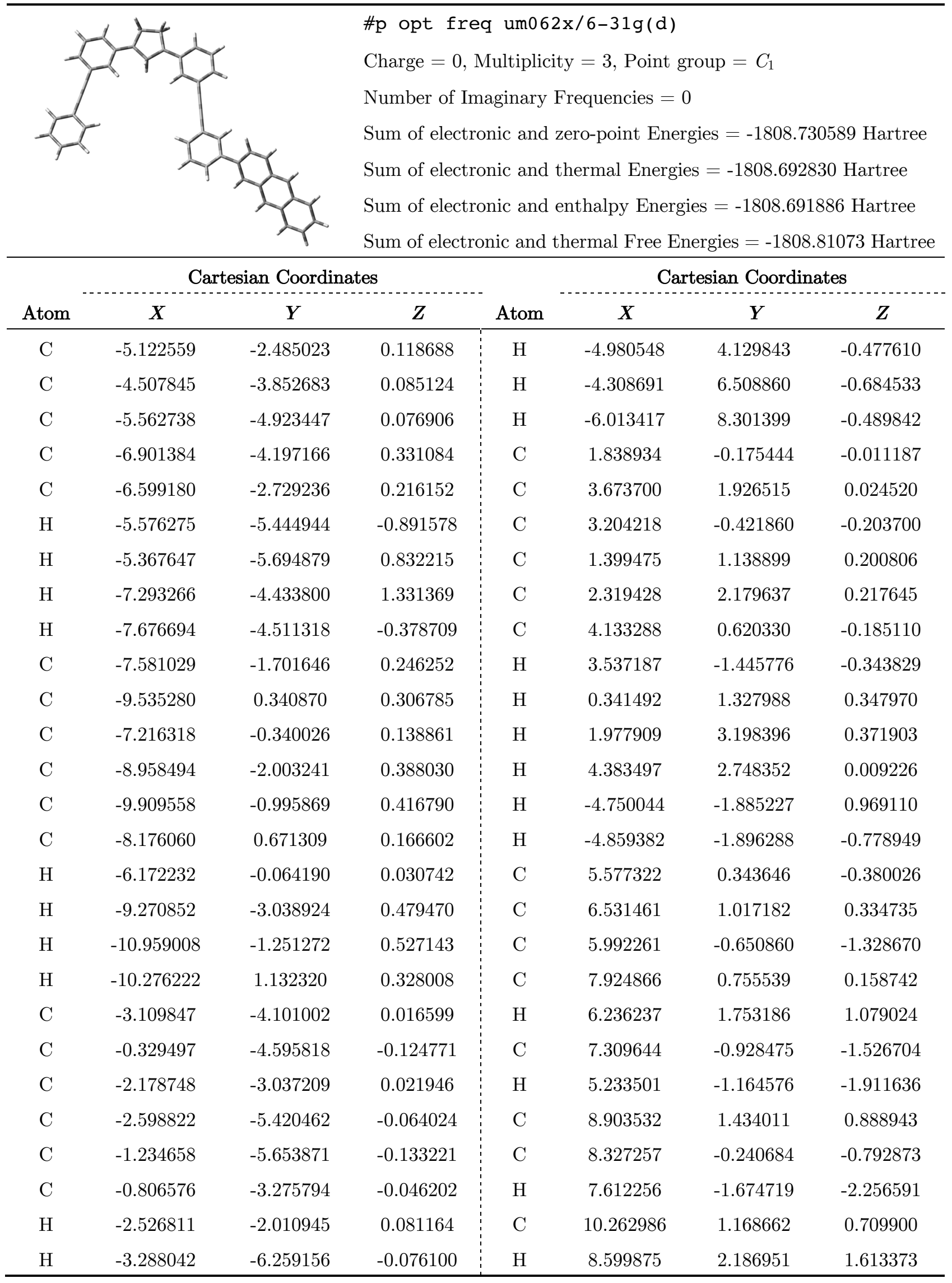




\begin{tabular}{cccccccc}
\hline & \multicolumn{3}{c}{ Cartesian Coordinates } & \multicolumn{4}{c}{ Cartesian Coordinates } \\
Atom & $\boldsymbol{X}$ & $\boldsymbol{Y}$ & $\boldsymbol{Z}$ & Atom & $\boldsymbol{X}$ & $\boldsymbol{Y}$ & $\boldsymbol{Z}$ \\
\hline $\mathrm{H}$ & -0.866831 & -6.673456 & -0.195414 & $\mathrm{C}$ & 9.685149 & -0.505697 & -0.973609 \\
$\mathrm{H}$ & 0.738874 & -4.773673 & -0.177554 & $\mathrm{C}$ & 11.275401 & 1.857393 & 1.450357 \\
$\mathrm{C}$ & -7.778859 & 2.042934 & 0.051185 & $\mathrm{C}$ & 10.664464 & 0.174867 & -0.245784 \\
$\mathrm{C}$ & -7.448640 & 3.204240 & -0.048080 & $\mathrm{H}$ & 9.988291 & -1.259929 & -1.696952 \\
$\mathrm{C}$ & 0.116476 & -2.180164 & -0.035159 & $\mathrm{C}$ & 12.595360 & 1.579028 & 1.255722 \\
$\mathrm{C}$ & 0.904562 & -1.260289 & -0.024343 & $\mathrm{H}$ & 10.966211 & 2.608377 & 2.172623 \\
$\mathrm{C}$ & -7.060923 & 4.577350 & -0.166392 & $\mathrm{C}$ & 12.060089 & -0.086855 & -0.419846 \\
$\mathrm{C}$ & -6.306325 & 7.260115 & -0.399252 & $\mathrm{C}$ & 12.994316 & 0.590986 & 0.305156 \\
$\mathrm{C}$ & -8.019332 & 5.595626 & -0.057734 & $\mathrm{H}$ & 13.354443 & 2.108028 & 1.823417 \\
$\mathrm{C}$ & -5.719853 & 4.919565 & -0.393117 & $\mathrm{H}$ & 12.357832 & -0.840105 & -1.144574 \\
$\mathrm{C}$ & -5.348936 & 6.253469 & -0.508355 & $\mathrm{H}$ & 14.050540 & 0.383767 & 0.164717 \\
$\mathrm{C}$ & -7.640524 & 6.927181 & -0.173880 & $\mathrm{H}$ & -8.389289 & 7.708473 & -0.088303 \\
$\mathrm{H}$ & -9.056120 & 5.328047 & 0.117757 & & & & \\
\hline
\end{tabular}




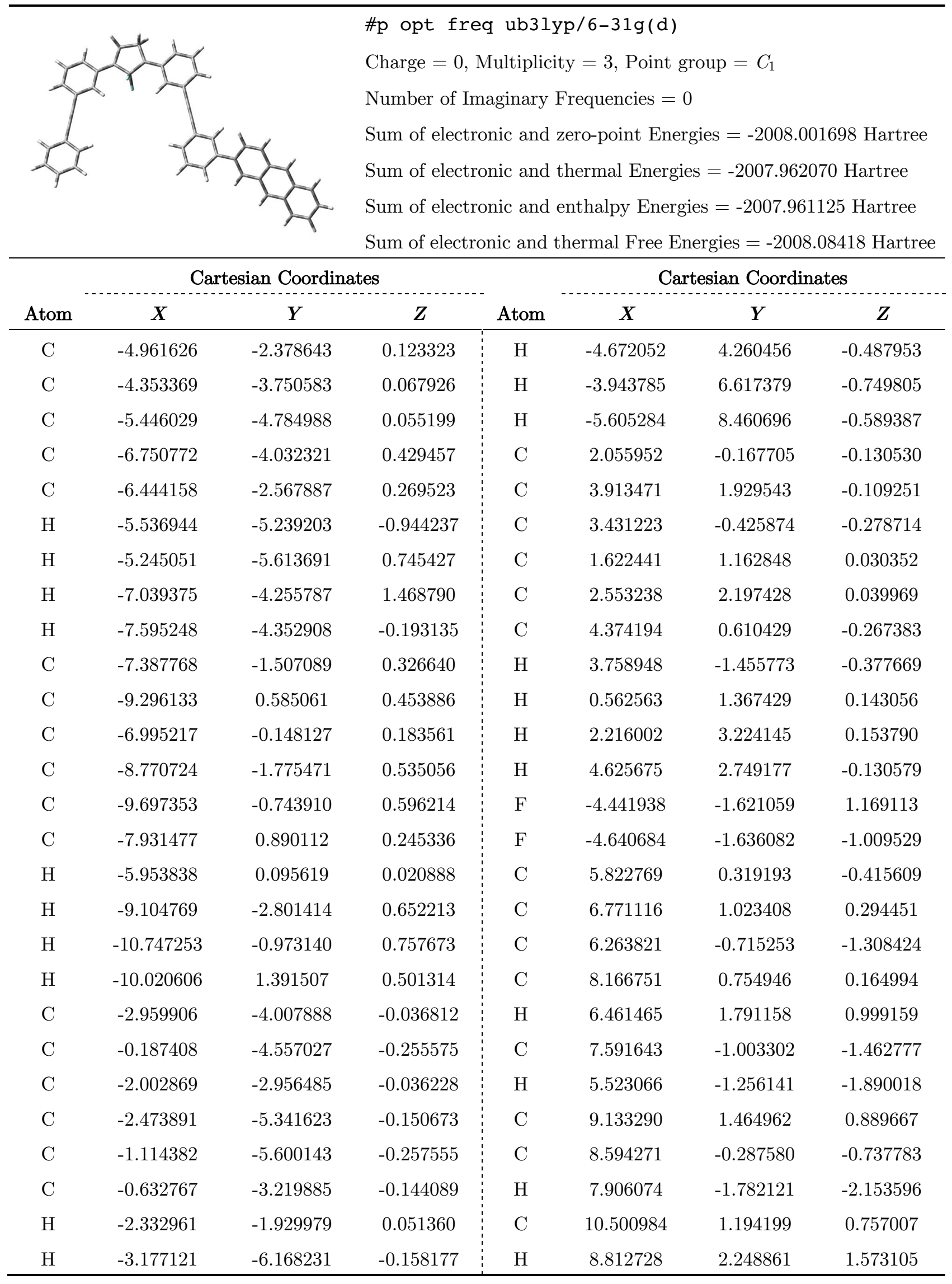




\begin{tabular}{cccccccc}
\hline & \multicolumn{3}{c}{ Cartesian Coordinates } & & \multicolumn{3}{c}{ Cartesian Coordinates } \\
Atom & $\boldsymbol{X}$ & \multicolumn{1}{c}{$\boldsymbol{Y}$} & $\boldsymbol{Z}$ & Atom & $\boldsymbol{X}$ & $\boldsymbol{Y}$ & $\boldsymbol{Z}$ \\
\hline $\mathrm{H}$ & -0.767386 & -6.626251 & -0.344587 & $\mathrm{C}$ & 9.960358 & -0.558611 & -0.872378 \\
$\mathrm{H}$ & 0.875504 & -4.759026 & -0.338429 & $\mathrm{C}$ & 11.496486 & 1.913277 & 1.489687 \\
$\mathrm{C}$ & -7.514224 & 2.245699 & 0.097686 & $\mathrm{C}$ & 10.927534 & 0.153359 & -0.150550 \\
$\mathrm{C}$ & -7.165014 & 3.403722 & -0.028645 & $\mathrm{H}$ & 10.280260 & -1.343466 & -1.555085 \\
$\mathrm{C}$ & 0.308610 & -2.148635 & -0.140223 & $\mathrm{C}$ & 12.827516 & 1.626252 & 1.338568 \\
$\mathrm{C}$ & 1.116378 & -1.239685 & -0.136347 & $\mathrm{H}$ & 11.173447 & 2.695876 & 2.172236 \\
$\mathrm{C}$ & -6.748053 & 4.758249 & -0.178274 & $\mathrm{C}$ & 12.326155 & -0.113325 & -0.277818 \\
$\mathrm{C}$ & -5.923954 & 7.428266 & -0.474640 & $\mathrm{C}$ & 13.248105 & 0.599395 & 0.442862 \\
$\mathrm{C}$ & -7.681276 & 5.810696 & -0.089410 & $\mathrm{H}$ & 13.572857 & 2.181018 & 1.902027 \\
$\mathrm{C}$ & -5.394146 & 5.068103 & -0.418380 & $\mathrm{H}$ & 12.644386 & -0.897024 & -0.961385 \\
$\mathrm{C}$ & -4.990672 & 6.392345 & -0.564610 & $\mathrm{H}$ & 14.308139 & 0.385735 & 0.335907 \\
$\mathrm{C}$ & -7.268478 & 7.131969 & -0.236794 & $\mathrm{H}$ & -7.998427 & 7.933910 & -0.165956 \\
$\mathrm{H}$ & -8.725265 & 5.576932 & 0.095444 & & & & \\
\hline
\end{tabular}




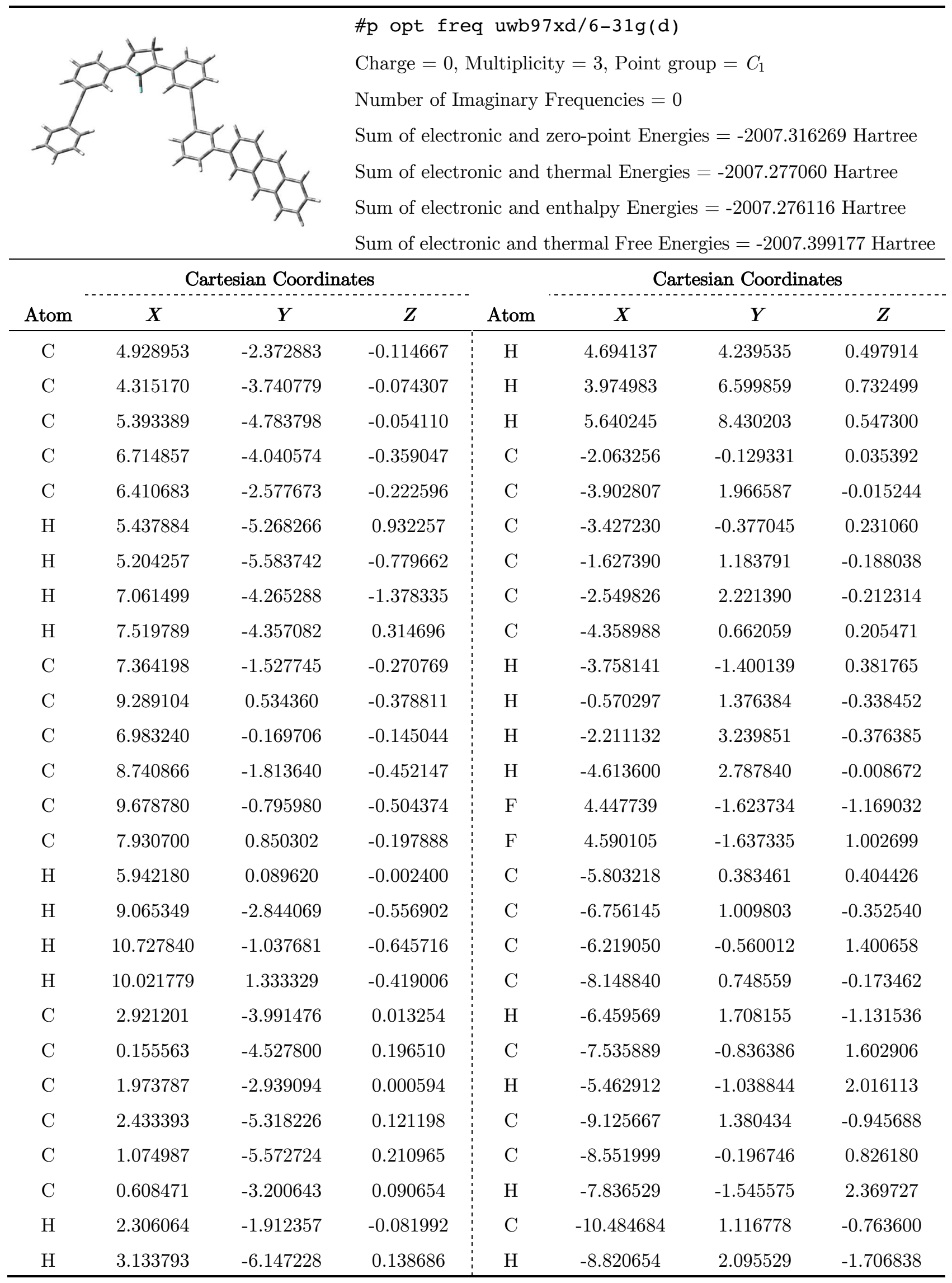




\begin{tabular}{cccccccc}
\hline & \multicolumn{3}{c}{ Cartesian Coordinates } & \multicolumn{4}{c}{ Cartesian Coordinates } \\
Atom & $\boldsymbol{X}$ & $\boldsymbol{Y}$ & $\boldsymbol{Z}$ & Atom & $\boldsymbol{X}$ & $\boldsymbol{Y}$ & $\boldsymbol{Z}$ \\
\hline $\mathrm{H}$ & 0.723620 & -6.596685 & 0.294174 & $\mathrm{C}$ & -9.909593 & -0.460375 & 1.009939 \\
$\mathrm{H}$ & -0.909049 & -4.724315 & 0.265504 & $\mathrm{C}$ & -11.495278 & 1.758190 & -1.546974 \\
$\mathrm{C}$ & 7.521978 & 2.214936 & -0.066534 & $\mathrm{C}$ & -10.887292 & 0.173381 & 0.239848 \\
$\mathrm{C}$ & 7.177621 & 3.369852 & 0.045700 & $\mathrm{H}$ & -10.213797 & -1.176723 & 1.770274 \\
$\mathrm{C}$ & -0.330281 & -2.121263 & 0.073317 & $\mathrm{C}$ & -12.814942 & 1.482255 & -1.347829 \\
$\mathrm{C}$ & -1.126075 & -1.209605 & 0.056613 & $\mathrm{H}$ & -11.187309 & 2.472077 & -2.306584 \\
$\mathrm{C}$ & 6.764323 & 4.732498 & 0.180135 & $\mathrm{C}$ & -12.282388 & -0.087419 & 0.416608 \\
$\mathrm{C}$ & 5.954666 & 7.395988 & 0.444504 & $\mathrm{C}$ & -13.214892 & 0.544844 & -0.350326 \\
$\mathrm{C}$ & 7.699801 & 5.771847 & 0.076918 & $\mathrm{H}$ & -13.572643 & 1.975716 & -1.949082 \\
$\mathrm{C}$ & 5.417913 & 5.044168 & 0.417479 & $\mathrm{H}$ & -12.583904 & -0.802583 & 1.177628 \\
$\mathrm{C}$ & 5.019791 & 6.368574 & 0.548298 & $\mathrm{H}$ & -14.271202 & 0.338371 & -0.206546 \\
$\mathrm{C}$ & 7.293798 & 7.093754 & 0.208732 & $\mathrm{H}$ & 8.025800 & 7.891616 & 0.127104 \\
$\mathrm{H}$ & 8.741911 & 5.530785 & -0.106841 & & & & \\
\hline
\end{tabular}




\section{DR4F1}

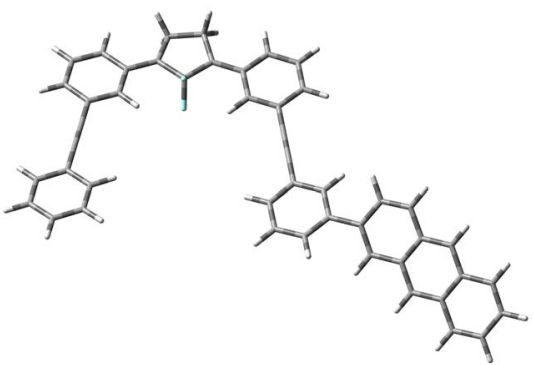

\#p opt freq um062x/6-31g(d)

Charge $=0$, Multiplicity $=3$, Point group $=C_{1}$

Number of Imaginary Frequencies $=0$

Sum of electronic and zero-point Energies $=-2007.165501$ Hartree

Sum of electronic and thermal Energies $=-2007.126185$ Hartree

Sum of electronic and enthalpy Energies = -2007.125241 Hartree

Sum of electronic and thermal Free Energies $=-2007.247690$ Hartree

\begin{tabular}{|c|c|c|c|c|c|c|c|}
\hline \multirow[b]{2}{*}{ Atom } & \multicolumn{3}{|c|}{ Cartesian Coordinates } & \multirow[b]{2}{*}{ Atom } & \multicolumn{3}{|c|}{ Cartesian Coordinates } \\
\hline & $X$ & $\boldsymbol{Y}$ & $Z$ & & $\boldsymbol{X}$ & $\boldsymbol{Y}$ & $Z$ \\
\hline $\mathrm{C}$ & 4.972909 & -2.363773 & -0.108510 & $\mathrm{H}$ & 4.520695 & 4.221811 & 0.534856 \\
\hline $\mathrm{C}$ & 4.388333 & -3.743217 & -0.083986 & $\mathrm{H}$ & 3.726347 & 6.562509 & 0.751894 \\
\hline $\mathrm{C}$ & 5.482328 & -4.768160 & -0.051628 & $\mathrm{H}$ & 5.328986 & 8.442890 & 0.523116 \\
\hline $\mathrm{C}$ & 6.796813 & -3.997234 & -0.328793 & $\mathrm{C}$ & -2.015523 & -0.170760 & -0.008657 \\
\hline $\mathrm{C}$ & 6.457954 & -2.542660 & -0.195365 & $\mathrm{C}$ & -3.865829 & 1.915899 & -0.064642 \\
\hline $\mathrm{H}$ & 5.518046 & -5.258956 & 0.931680 & $\mathrm{C}$ & -3.377862 & -0.425667 & 0.192406 \\
\hline $\mathrm{H}$ & 5.318264 & -5.565611 & -0.785566 & $\mathrm{C}$ & -1.586302 & 1.143818 & -0.239578 \\
\hline $\mathrm{H}$ & 7.169074 & -4.212431 & -1.340802 & $\mathrm{C}$ & -2.514338 & 2.177050 & -0.266422 \\
\hline $\mathrm{H}$ & 7.594743 & -4.296560 & 0.360562 & $\mathrm{C}$ & -4.314881 & 0.609211 & 0.163760 \\
\hline $\mathrm{C}$ & 7.380908 & -1.462340 & -0.232690 & $\mathrm{H}$ & -3.702559 & -1.450122 & 0.347329 \\
\hline $\mathrm{C}$ & 9.231147 & 0.661864 & -0.315190 & $\mathrm{H}$ & -0.530234 & 1.338337 & -0.393066 \\
\hline $\mathrm{C}$ & 6.946070 & -0.121279 & -0.120165 & $\mathrm{H}$ & -2.181262 & 3.196263 & -0.435229 \\
\hline $\mathrm{C}$ & 8.766919 & -1.704051 & -0.388732 & $\mathrm{H}$ & -4.581849 & 2.732439 & -0.057149 \\
\hline $\mathrm{C}$ & 9.670395 & -0.653916 & -0.428644 & $\mathrm{~F}$ & 4.492376 & -1.618996 & -1.161065 \\
\hline $\mathrm{C}$ & 7.859352 & 0.930762 & -0.160052 & $\mathrm{~F}$ & 4.604435 & -1.640771 & 1.002755 \\
\hline $\mathrm{H}$ & 5.892130 & 0.098008 & 0.000283 & $\mathrm{C}$ & -5.756191 & 0.325179 & 0.367927 \\
\hline $\mathrm{H}$ & 9.123748 & -2.725097 & -0.482568 & $\mathrm{C}$ & -6.717500 & 0.983248 & -0.351636 \\
\hline $\mathrm{H}$ & 10.729410 & -0.858690 & -0.550166 & $\mathrm{C}$ & -6.161031 & -0.659213 & 1.331343 \\
\hline $\mathrm{H}$ & 9.933607 & 1.487630 & -0.344760 & $\mathrm{C}$ & -8.108517 & 0.715879 & -0.165893 \\
\hline $\mathrm{C}$ & 2.993481 & -4.006507 & -0.010287 & $\mathrm{H}$ & -6.429916 & 1.711157 & -1.106792 \\
\hline $\mathrm{C}$ & 0.233446 & -4.555403 & 0.146331 & $\mathrm{C}$ & -7.475873 & -0.942033 & 1.538833 \\
\hline $\mathrm{C}$ & 2.046535 & -2.956157 & -0.024926 & $\mathrm{H}$ & -5.396679 & -1.160445 & 1.917870 \\
\hline $\mathrm{C}$ & 2.515177 & -5.335361 & 0.085422 & $\mathrm{C}$ & -9.094344 & 1.379272 & -0.900280 \\
\hline $\mathrm{C}$ & 1.156391 & -5.596988 & 0.162452 & $\mathrm{C}$ & -8.500826 & -0.269878 & 0.800683 \\
\hline $\mathrm{C}$ & 0.681303 & -3.225498 & 0.051923 & $\mathrm{H}$ & -7.770874 & -1.680220 & 2.279949 \\
\hline $\mathrm{H}$ & 2.379260 & -1.927852 & -0.097701 & $\mathrm{C}$ & -10.451444 & 1.109019 & -0.711091 \\
\hline $\mathrm{H}$ & 3.223565 & -6.157880 & 0.103217 & $\mathrm{H}$ & -8.798245 & 2.124280 & -1.635953 \\
\hline
\end{tabular}




\begin{tabular}{cccc|cccc}
\hline & \multicolumn{3}{c}{ Cartesian Coordinates } & \multicolumn{4}{c}{ Cartesian Coordinates } \\
Atom & $\boldsymbol{X}$ & $\boldsymbol{Y}$ & $\boldsymbol{Z}$ & Atom & $\boldsymbol{X}$ & $\boldsymbol{Y}$ & $\boldsymbol{Z}$ \\
\hline $\mathrm{H}$ & 0.808571 & -6.622513 & 0.236776 & $\mathrm{C}$ & -9.856378 & -0.539887 & 0.991429 \\
$\mathrm{H}$ & -0.831118 & -4.753989 & 0.205184 & $\mathrm{C}$ & -11.471154 & 1.782641 & -1.455462 \\
$\mathrm{C}$ & 7.403725 & 2.283441 & -0.041293 & $\mathrm{C}$ & -10.842878 & 0.125768 & 0.259536 \\
$\mathrm{C}$ & 7.023499 & 3.428838 & 0.060989 & $\mathrm{H}$ & -10.151957 & -1.286064 & 1.726174 \\
$\mathrm{C}$ & -0.265522 & -2.150839 & 0.032854 & $\mathrm{C}$ & -12.788638 & 1.499944 & -1.250600 \\
$\mathrm{C}$ & -1.070247 & -1.245925 & 0.014715 & $\mathrm{H}$ & -11.169479 & 2.525726 & -2.188993 \\
$\mathrm{C}$ & 6.567625 & 4.780341 & 0.184329 & $\mathrm{C}$ & -12.236228 & -0.140962 & 0.443958 \\
$\mathrm{C}$ & 5.675390 & 7.418549 & 0.428136 & $\mathrm{C}$ & -13.177622 & 0.522367 & -0.285196 \\
$\mathrm{C}$ & 7.468383 & 5.847598 & 0.056393 & $\mathrm{H}$ & -13.553297 & 2.017529 & -1.821323 \\
$\mathrm{C}$ & 5.214483 & 5.050128 & 0.435790 & $\mathrm{H}$ & -12.526414 & -0.886127 & 1.180027 \\
$\mathrm{C}$ & 4.775223 & 6.362569 & 0.556420 & $\mathrm{H}$ & -14.232026 & 0.311514 & -0.136706 \\
$\mathrm{C}$ & 7.021092 & 7.157256 & 0.178018 & $\mathrm{H}$ & 7.725138 & 7.977351 & 0.077478 \\
$\mathrm{H}$ & 8.514525 & 5.635404 & -0.138390 & & & & \\
\hline
\end{tabular}


DR5a

\begin{tabular}{|c|c|c|c|c|c|c|c|}
\hline \multirow[b]{3}{*}{ Atoms } & & & \multicolumn{5}{|c|}{$\begin{array}{l}\text { Sum of electronic and zero-point Energies }=-2008.002475 \text { Hartree } \\
\text { Sum of electronic and thermal Energies }=-2007.962851 \text { Hartree } \\
\text { Sum of electronic and thermal Enthalpies }=-2007.961906 \text { Hartree } \\
\text { Sum of electronic and thermal Free Energies }=-2008.084694 \text { Hartree }\end{array}$} \\
\hline & \multicolumn{3}{|c|}{ Cartesian Coordinates } & & \multicolumn{3}{|c|}{ Cartesian Coordinates } \\
\hline & $X$ & $Y$ & $Z$ & Atoms & $X$ & $Y$ & $Z$ \\
\hline $\mathrm{C}$ & 5.557422 & -1.813470 & -0.047041 & $\mathrm{C}$ & 7.012573 & -1.445172 & -0.001151 \\
\hline $\mathrm{C}$ & 7.844758 & -2.695621 & 0.088229 & $\mathrm{C}$ & 6.883995 & -3.871153 & -0.233982 \\
\hline $\mathrm{C}$ & 5.492145 & -3.312374 & -0.113257 & $\mathrm{H}$ & 8.703361 & -2.676220 & -0.594330 \\
\hline $\mathrm{H}$ & 8.265175 & -2.808850 & 1.100130 & $\mathrm{H}$ & 7.051178 & -4.725016 & 0.434193 \\
\hline $\mathrm{H}$ & 7.063131 & -4.245801 & -1.254306 & $\mathrm{C}$ & 4.286358 & -4.063673 & -0.138836 \\
\hline $\mathrm{C}$ & 1.900132 & -5.592790 & -0.201903 & $\mathrm{C}$ & 3.013499 & -3.438963 & -0.035531 \\
\hline $\mathrm{C}$ & 4.316174 & -5.481044 & -0.273899 & $\mathrm{C}$ & 3.142062 & -6.220769 & -0.304273 \\
\hline $\mathrm{C}$ & 1.831071 & -4.187133 & -0.065810 & $\mathrm{H}$ & 2.949951 & -2.364343 & 0.071660 \\
\hline $\mathrm{H}$ & 5.270831 & -5.990208 & -0.359007 & $\mathrm{H}$ & 3.189935 & -7.301260 & -0.409848 \\
\hline $\mathrm{H}$ & 0.982271 & -6.171110 & -0.225103 & $\mathrm{C}$ & 7.503252 & -0.112151 & 0.034078 \\
\hline $\mathrm{C}$ & 8.515688 & 2.534272 & 0.115884 & $\mathrm{C}$ & 6.630968 & 1.008217 & -0.038326 \\
\hline $\mathrm{C}$ & 8.899204 & 0.145398 & 0.148190 & $\mathrm{C}$ & 9.384981 & 1.444988 & 0.187957 \\
\hline $\mathrm{C}$ & 7.123295 & 2.317565 & 0.001421 & $\mathrm{H}$ & 5.564169 & 0.853049 & -0.128915 \\
\hline $\mathrm{H}$ & 9.593374 & -0.686601 & 0.209698 & $\mathrm{H}$ & 10.454303 & 1.616781 & 0.277111 \\
\hline $\mathrm{H}$ & 8.895516 & 3.550275 & 0.146468 & $\mathrm{C}$ & 0.566234 & -3.538315 & 0.041874 \\
\hline $\mathrm{C}$ & -0.515631 & -2.989976 & 0.135401 & $\mathrm{C}$ & 6.228925 & 3.425627 & -0.074812 \\
\hline $\mathrm{C}$ & 5.471376 & 4.374656 & -0.141053 & $\mathrm{C}$ & 4.577072 & 5.481437 & -0.220122 \\
\hline $\mathrm{C}$ & 2.812069 & 7.662236 & -0.377598 & $\mathrm{C}$ & 5.061579 & 6.802803 & -0.144759 \\
\hline $\mathrm{C}$ & 3.191826 & 5.272686 & -0.375863 & $\mathrm{C}$ & 2.321357 & 6.356220 & -0.453519 \\
\hline $\mathrm{C}$ & 4.183480 & 7.880155 & -0.223119 & $\mathrm{H}$ & 6.127821 & 6.968575 & -0.024823 \\
\hline $\mathrm{H}$ & 2.814422 & 4.256502 & -0.434933 & $\mathrm{H}$ & 1.255649 & 6.181262 & -0.574175 \\
\hline $\mathrm{H}$ & 4.570356 & 8.893929 & -0.163606 & $\mathrm{H}$ & 2.129568 & 8.505495 & -0.438715 \\
\hline $\mathrm{C}$ & -1.777158 & -2.340742 & 0.246671 & $\mathrm{C}$ & -4.293291 & -1.043547 & 0.473645 \\
\hline $\mathrm{C}$ & -1.857619 & -0.946656 & 0.436014 & $\mathrm{C}$ & -2.978615 & -3.074275 & 0.174977 \\
\hline $\mathrm{C}$ & -4.206436 & -2.435599 & 0.288099 & $\mathrm{C}$ & -3.090680 & -0.317131 & 0.548757 \\
\hline $\mathrm{H}$ & -0.941748 & -0.365963 & 0.486215 & $\mathrm{H}$ & -2.934278 & -4.150865 & 0.041781 \\
\hline $\mathrm{H}$ & -5.117063 & -3.026946 & 0.260137 & $\mathrm{H}$ & -3.123385 & 0.761836 & 0.669493 \\
\hline
\end{tabular}




\begin{tabular}{cccc|cccc}
\hline & \multicolumn{3}{c}{ Cartesian Coordinates } & & \multicolumn{3}{c}{ Cartesian Coordinates } \\
Atoms & $\boldsymbol{X}$ & $\boldsymbol{Y}$ & $\boldsymbol{Z}$ & Atoms & $\boldsymbol{X}$ & $\boldsymbol{Y}$ & $\boldsymbol{Z}$ \\
\hline $\mathrm{C}$ & -5.605947 & -0.364607 & 0.583463 & $\mathrm{H}$ & -6.591861 & -1.606939 & -0.853365 \\
$\mathrm{C}$ & -6.694630 & -0.781435 & -0.153551 & $\mathrm{C}$ & -6.967836 & 1.400870 & 1.587272 \\
$\mathrm{C}$ & -7.963754 & -0.136434 & -0.060711 & $\mathrm{C}$ & -5.768119 & 0.756364 & 1.467193 \\
$\mathrm{C}$ & -8.110046 & 0.987772 & 0.833496 & $\mathrm{C}$ & -9.069478 & -0.554016 & -0.813660 \\
$\mathrm{H}$ & -4.923718 & 1.073632 & 2.071904 & $\mathrm{H}$ & -9.458685 & 2.472654 & 1.608717 \\
$\mathrm{H}$ & -7.072642 & 2.238873 & 2.272422 & $\mathrm{C}$ & -10.311678 & 0.085560 & -0.717001 \\
$\mathrm{H}$ & -8.960397 & -1.398976 & -1.490767 & $\mathrm{C}$ & -11.447350 & -0.332702 & -1.478709 \\
$\mathrm{C}$ & -10.457458 & 1.207647 & 0.182233 & $\mathrm{H}$ & -11.837157 & 2.695841 & 0.950113 \\
$\mathrm{C}$ & -9.350465 & 1.627008 & 0.932263 & $\mathrm{C}$ & -12.650352 & 0.312461 & -1.362541 \\
$\mathrm{H}$ & -11.335551 & -1.177028 & -2.154940 & $\mathrm{H}$ & -13.504793 & -0.016495 & -1.947770 \\
$\mathrm{C}$ & -12.793904 & 1.419328 & -0.475024 & $\mathrm{H}$ & -13.755726 & 1.918712 & -0.395920 \\
$\mathrm{C}$ & -11.730224 & 1.851808 & 0.272702 & $\mathrm{~F}$ & 4.872661 & -1.310698 & 1.055657 \\
$\mathrm{~F}$ & 4.906344 & -1.230056 & -1.130338 & & & & \\
\hline
\end{tabular}




\begin{tabular}{|c|c|c|c|c|c|c|c|}
\hline \multirow[b]{3}{*}{ Atoms } & & & \multicolumn{5}{|c|}{$\begin{array}{l}\text { Sum of electronic and zero-point Energies }=-2008.009636 \text { Hartree } \\
\text { Sum of electronic and thermal Energies }=-2007.970060 \text { Hartree } \\
\text { Sum of electronic and thermal Enthalpies }=-2007.969116 \text { Hartree } \\
\text { Sum of electronic and thermal Free Energies }=-2008.091682 \text { Hartree }\end{array}$} \\
\hline & \multicolumn{3}{|c|}{ Cartesian Coordinates } & & \multicolumn{3}{|c|}{ Cartesian Coordinates } \\
\hline & $X$ & $Y$ & $Z$ & Atoms & $X$ & $Y$ & $Z$ \\
\hline $\mathrm{C}$ & -3.841504 & -1.808658 & 0.071967 & $\mathrm{C}$ & -5.277740 & -2.247501 & 0.087307 \\
\hline $\mathrm{C}$ & -5.342788 & -3.751879 & 0.071005 & $\mathrm{C}$ & -3.904492 & -4.243928 & 0.380992 \\
\hline $\mathrm{C}$ & -3.006460 & -3.052847 & 0.174838 & $\mathrm{H}$ & -6.069103 & -4.147565 & 0.791395 \\
\hline $\mathrm{H}$ & -5.667165 & -4.114159 & -0.917119 & $\mathrm{H}$ & -3.623498 & -5.094818 & -0.251517 \\
\hline $\mathrm{H}$ & -3.837814 & -4.603001 & 1.419974 & $\mathrm{C}$ & -1.597469 & -3.068567 & 0.150112 \\
\hline $\mathrm{C}$ & 1.258272 & -3.134936 & 0.113495 & $\mathrm{C}$ & -0.824099 & -1.877133 & -0.026207 \\
\hline $\mathrm{C}$ & -0.873315 & -4.291055 & 0.306956 & $\mathrm{C}$ & 0.504876 & -4.322710 & 0.289286 \\
\hline $\mathrm{C}$ & 0.553225 & -1.914146 & -0.042865 & $\mathrm{H}$ & -1.333466 & -0.930017 & -0.150189 \\
\hline $\mathrm{H}$ & -1.420161 & -5.218112 & 0.447567 & $\mathrm{H}$ & 1.030746 & -5.264701 & 0.411853 \\
\hline $\mathrm{H}$ & 1.118615 & -0.997205 & -0.178102 & $\mathrm{C}$ & -6.377799 & -1.367494 & 0.050694 \\
\hline $\mathrm{C}$ & -8.629884 & 0.388458 & -0.032344 & $\mathrm{C}$ & -6.217132 & 0.054794 & 0.042624 \\
\hline $\mathrm{C}$ & -7.717097 & -1.866061 & 0.014929 & $\mathrm{C}$ & -8.803632 & -1.018404 & -0.025246 \\
\hline $\mathrm{C}$ & -7.306404 & 0.897640 & 0.002220 & $\mathrm{H}$ & -5.219437 & 0.473970 & 0.070183 \\
\hline $\mathrm{H}$ & -7.884879 & -2.938505 & 0.015335 & $\mathrm{H}$ & -9.810787 & -1.423093 & -0.053118 \\
\hline $\mathrm{H}$ & -7.159598 & 1.973352 & -0.003596 & $\mathrm{C}$ & 2.671913 & -3.167444 & 0.095140 \\
\hline $\mathrm{C}$ & 3.890941 & -3.195078 & 0.079007 & $\mathrm{C}$ & -9.744171 & 1.257980 & -0.073039 \\
\hline $\mathrm{C}$ & -10.704741 & 2.008588 & -0.108003 & $\mathrm{C}$ & 5.312875 & -3.226997 & 0.061971 \\
\hline $\mathrm{C}$ & 8.114141 & -3.276844 & 0.007457 & $\mathrm{C}$ & 6.049155 & -2.039946 & -0.115340 \\
\hline $\mathrm{C}$ & 6.005360 & -4.446215 & 0.211281 & $\mathrm{C}$ & 7.395897 & -4.460037 & 0.181781 \\
\hline $\mathrm{C}$ & 7.449624 & -2.045964 & -0.139769 & $\mathrm{H}$ & 5.511107 & -1.108700 & -0.259664 \\
\hline $\mathrm{H}$ & 5.443128 & -5.364840 & 0.345452 & $\mathrm{H}$ & 7.928028 & -5.399682 & 0.302992 \\
\hline $\mathrm{H}$ & 9.199921 & -3.302430 & 0.012579 & $\mathrm{C}$ & -11.823836 & 2.884895 & -0.148683 \\
\hline $\mathrm{C}$ & -14.035435 & 4.617454 & -0.229018 & $\mathrm{C}$ & -11.640403 & 4.283660 & -0.151342 \\
\hline $\mathrm{C}$ & -13.137219 & 2.371396 & -0.187082 & $\mathrm{C}$ & -14.228927 & 3.233375 & -0.226794 \\
\hline $\mathrm{C}$ & -12.738691 & 5.137228 & -0.191180 & $\mathrm{H}$ & -10.631651 & 4.683799 & -0.121908 \\
\hline $\mathrm{H}$ & -13.283721 & 1.295684 & -0.185258 & $\mathrm{H}$ & -15.235331 & 2.824542 & -0.256169 \\
\hline $\mathrm{H}$ & -12.583219 & 6.212705 & -0.192770 & $\mathrm{H}$ & -14.890373 & 5.287238 & -0.260101 \\
\hline
\end{tabular}




\begin{tabular}{cccc|cccc}
\hline & \multicolumn{3}{c}{ Cartesian Coordinates } & & \multicolumn{3}{c}{ Cartesian Coordinates } \\
Atoms & $\boldsymbol{X}$ & $\boldsymbol{Y}$ & $\boldsymbol{Z}$ & Atoms & $\boldsymbol{X}$ & $\boldsymbol{Y}$ & $\boldsymbol{Z}$ \\
\cline { 2 - 8 } $\mathrm{C}$ & 8.212266 & -0.783722 & -0.313929 & $\mathrm{H}$ & 6.907338 & 0.401565 & 0.897790 \\
$\mathrm{C}$ & 7.796568 & 0.392159 & 0.272448 & $\mathrm{C}$ & 10.135118 & 0.377717 & -1.277093 \\
$\mathrm{C}$ & 8.520470 & 1.612044 & 0.116230 & $\mathrm{C}$ & 9.412797 & -0.770049 & -1.101388 \\
$\mathrm{C}$ & 9.725128 & 1.609807 & -0.679659 & $\mathrm{C}$ & 8.103654 & 2.808250 & 0.715225 \\
$\mathrm{H}$ & 9.731394 & -1.687595 & -1.587454 & $\mathrm{H}$ & 11.348954 & 2.796960 & -1.440931 \\
$\mathrm{H}$ & 11.034836 & 0.370230 & -1.887909 & $\mathrm{C}$ & 8.820802 & 4.000841 & 0.557503 \\
$\mathrm{H}$ & 7.197449 & 2.811082 & 1.317752 & $\mathrm{C}$ & 8.404092 & 5.227995 & 1.161956 \\
$\mathrm{C}$ & 10.024257 & 3.997846 & -0.242936 & $\mathrm{H}$ & 11.652447 & 5.216423 & -0.999613 \\
$\mathrm{C}$ & 10.442354 & 2.800604 & -0.838875 & $\mathrm{C}$ & 9.126203 & 6.379341 & 0.989462 \\
$\mathrm{H}$ & 7.498290 & 5.228044 & 1.763739 & $\mathrm{H}$ & 8.797434 & 7.304620 & 1.454772 \\
$\mathrm{C}$ & 10.313526 & 6.376125 & 0.199577 & $\mathrm{H}$ & 10.873243 & 7.298967 & 0.073777 \\
$\mathrm{C}$ & 10.747293 & 5.221368 & -0.396809 & $\mathrm{~F}$ & -3.556760 & -0.919230 & 1.105832 \\
$\mathrm{~F}$ & -3.546366 & -1.075932 & -1.075490 & & & & \\
\hline
\end{tabular}




\section{References}

[S1] Dennington, R.; Keith, T. A.; Millam, J. M. GaussView, Version 6.0.16; Semichem Inc.: Shawnee Mission, KS, 2016. 